\title{
An Implicit Finite Element Method for Discrete Dynamic Fracture
}

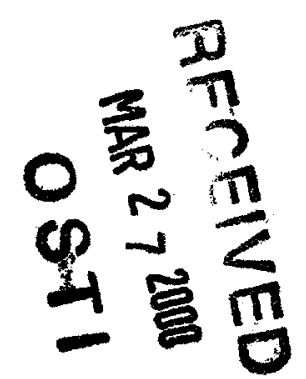

\section{Los Alamos \\ NATIONAL LABORATORY}

Los Alamos National Laboratory is operated by the University of California for the United States Department of Energy under contract W-7405-ENG-36. 
This thesis was accepted by the Department of Mechanical Engineering, Colorado State University, Fort Collins, Colorado, in partial fulfillment of the requirements for the degree of Master of Science. The text and illustrations are the independent work of the author and only the front matter has been edited by the CIC-1 Writing and Editing Staff to conform with Department of Energy and Los Alamos National Laboratory publication policies.

An Affirmative Action/Equal Opportunity Employer

This report was prepared as an account of work sponsored by an agency of the United States Government. Neither The Regents of the University of California, the United States Government nor any agency thereof, nor any of their employees, makes any warranty, express or implied, or assumes any legal liability or responsibility for the accuracy, completeness, or usefulness of any information, apparatus, product, or process disclosed, or represents that its use would not infringe privately owned rights. Reference herein to any specific commercial product, process, or service by trade name, trademark, manufacturer, or otherwise, does not necessarily constitute or imply its endorsement, recommendation, or favoring by The Regents of the University of California, the United States Government, or any agency thereof. The views and opinions of authors expressed herein do not necessarily state or reflect those of The Regents of the University of California, the United States Government, or any agency thereof. Los Alamos National Laboratory strongly supports academic freedom and a researcher's right to publish; as an institution, however, the Laboratory does not endorse the viewpoint of a publication or guarantee its technical correctness. 


\section{DISCLAIMER}

Portions of this document may be illegible in electronic image products. Images are produced from the best available original document. 
An Implicit Finite Element Method for Discrete Dynamic Fracture

Jobie M. Gerken 



\section{TABLE OF CONTENTS}

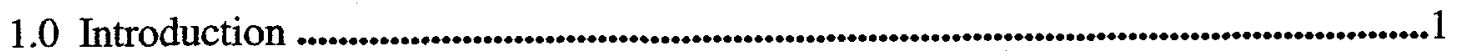

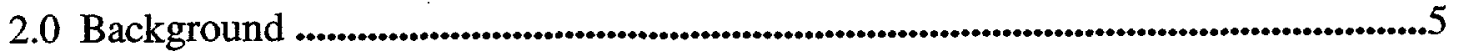

2.1 Finite Element Method ....................................................................................5

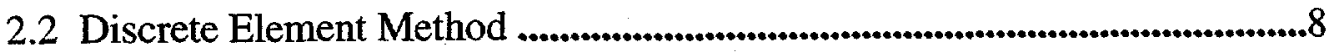

2.3 Element Free Galerkin Method ................................................................... 12

2.4 Molecular Dynamics .................................................................................13

2.5 Evaluation................................................................................................ 15

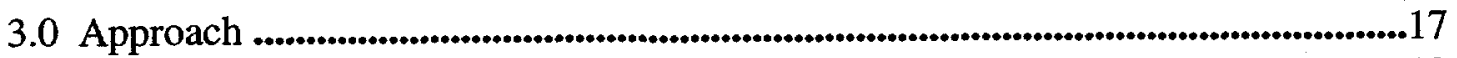

3.1 Defining an Interface Crack Element ............................................................18

3.2 Interface Crack ..........................................................................................20

3.3 Global Assembly and Analysis ..................................................................23

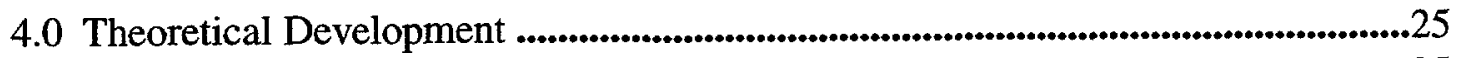

$4.1 \mathrm{Hu}$ - Washizu Energy Principle .................................................................25

4.2 Finite Element Equations ....................................................................27

4.3 Implementation of a 4 Node Plane Element ................................................30

4.3.1 Stiffness Matrix ...........................................................................30

4.3.2 Load Vector .........................................................................33

4.4 ABAQUS/Standard Implementation ....................................................40

4.4.1 User Element .........................................................................40

4.4.2 Multi-Point Constraint ..............................................................44

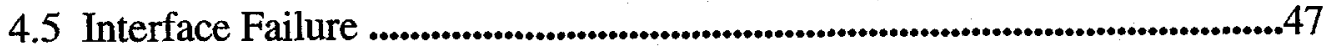

4.5.1 Convergence ...........................................................................47

4.5.2 Interface Failure ...................................................................4 48

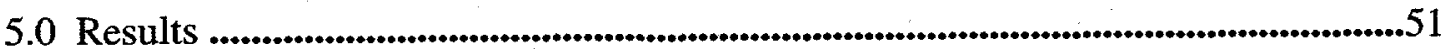

5.1 Structural Dynamics with Distributed Cracks ...............................................51

5.1.1 Axial Vibration of a Beam ......................................................52

5.1.2 Transverse Vibration of a Cantilevered Beam ............................55

5.1.3 Vibration of a Thin Ring ......................................................59

5.2 Fracture Modeling .................................................................................61

5.2.1 Compact Tension Specimen ...................................................662

5.2.2 Single Edge Notched Beam .......................................................668

5.3 A Future Application ................................................................................76 
7.0 References 


\title{
AN IMPLICIT FINITE ELEMENT METHOD FOR
}

\section{DISCRETE DYNAMIC FRACTURE}

by

Jobie M. Gerken

\begin{abstract}
This thesis presents a method to model dynamic crack propagation in structures. The method presented herein provides a means to address the three major challenges of modeling dynamic crack propagation which are; 1) reproducing the material behavior in the presence of fracture, 2) modeling the geometry changes that occur as a result of crack propagation, 3) predicting wave propagation effects on the fracture process.

Based on the finite element method, a new, two-dimensional, element is developed via the Hu-Washizu energy principle. The interface crack element (ICE) consists of two adjacent four-node plane elements with a crack at the element interface. The interface crack is represented by an externally applied strain field in the Hu-Washizu energy function. The results is a standard stiffness matrix and load vector that represent the structural components of the element and an additional term in the load vector that represents the interface crack component. Failure criteria are defined for the interface so that the interface can fail and separate to model crack propagation. The element is implemented as a User Element in the implicit integration finite element code ABAQUS/Standard. Using the implicit code ABAQUS/Standard allows the technique to model a range of time scales and provides
\end{abstract}


an efficient method of assembling the global equations and solving for the nodal variables.

In the absence of interface cracks, the ICE is shown to model the dynamic behavior of three simple structures within acceptable engineering accuracy. These simple structures are also analyzed with interface cracks present. Comparisons show an increase in compliance of the structures when interface cracks are included. The modeling of crack initiation and propagation are demonstrated by modeling Compact Tension and Single Edge Notched Beam specimens. The results of the simulations show the expected crack propagation patterns, but the failure loads and crack propagation speeds are well below the expected values. Application to the mechanically coupled cookoff test for high explosives has also been undertaken. The simulations show that, even with neglecting important material behaviors for which limited information exists, the general crack features of the experiments can be reproduced.

Jobie Michael Gerken

Department of Mechanical Engineering Colorado State University

Fort Collins, CO 80523

Fall 1998 


\section{Introduction}

There has been considerable interest in developing numerical models having the capability to predict the structural static and dynamic response of structures in the presence of failure by fracture of engineering materials. The major challenges that must be addressed in modeling of dynamic crack propagation are producing the correct material behavior in the presence of fracture, modeling geometry changes that are a result of crack propagation, and predicting wave propagation effects on the fracture process. Currently, there are computational tools available that adequately predict both static and dynamic structural response, but the existing capability to predict and model crack propagation on a structural scale is insufficient for practical engineering application.

Fracture in structures has been investigated using a number of different computational methods and techniques. The methods include most, if not all, of the well developed computational methods: finite difference, finite element, boundary integral equation (boundary element), element free Galerkin, molecular dynamic, and others [Liebowitz et al, 1995] [Aliabadi, 1995]. A large amount of effort, and the effort of this thesis, has been expended on fracture modeling using the finite element method (FEM) [Nishioka, 1997] [De Borst, 1997]. The techniques which have been used in FEM include, but are not limited to, techniques such as special element formulation to model crack tip singularities and discontinuities [Banks-Sills and 
Sherman, 1989], [Lotfi and Shing, 1995] [Sandhu and Huang, 1975], adaptive meshing [Nishioka et al, 1981], calculation of crack tip parameters [Banks-Sills and Sherman, 1992], [Li et al, 1985], [Parks, 1974], damage evolution [Lemaitre, 1986], discrete models [Hoff et al, 1986] [Liaw et al, 1984], and others. Some of these methods are cumbersome or inaccurate while others are inappropriate to use as engineering analysis tools in the modeling of discrete crack propagation.

The primary goal of this thesis is to present a useful numerical engineering analysis tool that can with relative ease model the dynamic response of structures in the presence of discrete fracture with reasonable engineering accuracy. In this context, "discrete fracture" modeling refers to the ability to model the behavior of multiple, stationary or propagating, discrete cracks throughout the structure. Discrete fracture, as described here, is distinct and different from damage models [Lemaitre, 1986] in which the presence of microscopic cracks is assumed to affect the macroscopic properties of the material. To be useful, the technique should satisfy the following requirements:

1. operates with mesh complexity similar to typical structural analysis,

2. uses fracture criteria based on accepted fracture mechanics theories and data,

3. able to model a variety of problems which are typical of problems commonly addressed using FEM,

4. able to model crack growth paths which are not predetermined,

5. able to model the behavior of many small cracks distributed throughout a structure. 
This tool will be potentially useful in modeling practical engineering problems in which structural dynamic behavior and fracture are important. Some of the potential applications include: predicting the onset of rapid fracture and the resulting crack propagation patterns of aircraft skins, modeling the failure of bonded joints, determining the predominant fracture patterns in the mechanically coupled cook-off testing of high explosive, predicting the crack propagation pattern of dynamically loaded cylindrical shells and spheres, and other applications.

Using the implicit integration FEM code ABAQUS (ABAQUS/Standard), the dynamic structural response of engineering systems can be determined. This thesis describes a technique by which the structural response is coupled with a model of discrete fracture by defining a user element in ABAQUS. A two dimensional structural model is constructed of 4 node 2-D solid elements. Each element interface is modeled as having a small crack present. Four nodes define each interface so that the element faces can separate if the interface crack fails. A hybrid, three field, finite element formulation is developed via the $\mathrm{Hu}$-Washizu energy principle $[\mathrm{Hu}, 1955]$ in which the interface crack is represented by an externally applied strain field. During the analysis, this crack can grow based on standard fracture mechanics criteria and it is driven by the averaged stress state at the interface. If the crack grows wider than the element interface or the failure stress of the material on the interface is exceeded, the interface separates to create new crack surface.

Based on this formulation, the technique will be able to model the dynamic behavior of a structure in the presence of a distribution of small cracks. It will also be able to model the failure by fracture resulting at any of the preexisting small cracks at 
the element interfaces. Because of the implicit integration of the nodal displacements and velocities in ABAQUS, this technique will be able to model a range of time scales. The analysis will therefore be able to take large time steps while the model remains linear (before fracture occurs) and transition to small time steps as the model becomes non-linear during crack propagation.

The modeling of discrete fracture and new surface creation could prove to be beneficial in many areas of computational mechanics, design, and analysis. It could provide not only the modeling of failure, but also provide information on a structural scale of crack initiation, propagation and catastrophic failure. The technique presented herein provides a simple method to model these aspects of crack growth. 


\section{Background}

The modeling of discrete fracture of materials has for some time been an elusive goal of the scientific community. There have been many attempts by several investigators, with varying results. An early development, and possibly the first [Li and Reed, 1995], is the work of Goodman et al [1968] in which the authors developed a special, zero thickness, interface finite element to model the quasi-static behavior of joints and seams in rock. The method allows for the propagation of cracks through these interface elements by reducing their stiffness contribution to zero after the normal stress fracture criterion has been exceeded. Work similar to that presented herein is a double noding technique developed for the finite element method by Liaw et al [1984]. This method allowed for crack propagation by separation along element interfaces along a predefined "double node path" based on the J integral fracture criterion.

Although the finite element method has by far been the most developed method of modeling discrete fracture, there have been many other methods employed. The following is a brief overview of some of the representative work that has been done to numerically model discrete fracture.

\subsection{Finite Element Method}

Needleman et al have developed a discrete dynamic crack propagation model for micro cracks in which continuum finite elements are bonded together by interfacial 
cohesion forces, [Needleman, 1987], [Needleman, 1992], [Xu and Needleman, 1993], [Xu and Needleman, 1994a]. The analyses use a material constitutive law that relates stress and strain to characterize the continuum elements and a relation between the traction and displacement jump between element nodes to characterize the interfacial cohesive forces.

The constitutive law relating stress to strain in the continuum is chosen to match the gross behavior of the material. The authors have presented results for models such as isotropic-hyperelastic [Xu and Needleman, 1994b], elastic-plastic [Xu and Needleman, 1993], and elastic-viscoplastic [Needleman, 1997], [Siegmund and Needleman, 1996] and [Siegmund, Fleck and Needleman, 1997].

The cohesive relation for the interface is such that

$$
\mathrm{T}=\frac{\partial \phi}{\partial \Delta}
$$

where $\mathrm{T}$ is the traction across the interface, $\phi$ is the potential, and $\Delta$ is the interfacial displacement. In their analysis, the interface is taken to be elastic so that before fracture any dissipation across the interface is neglected. The potential is chosen such that, given no tangential displacement, the normal traction, $T_{n}$, is a function of normal separation, $\Delta_{\mathrm{n}}$, as follows:

- $\mathrm{T}_{\mathrm{n}}$ tends to $-\infty$ for $\Delta_{\mathrm{n}}$ less than zero,

- $\quad T_{n}$ tends to zero for $\Delta_{n}$ greater than a critical normal separation, $\delta_{c r}{ }^{n}$,

- and $T_{n}$ is greater than zero for $0<\Delta_{n}<\delta_{c r}{ }^{n}$. 
For no normal displacement, the tangential traction, $T_{t}$, is a function of tangential displacement, $\Delta_{t}$, as follows:

- $T_{t}$ tends to zero for $\left|\Delta_{t}\right|>\delta_{c r}{ }^{t}$,

- $T_{t}$ is greater than zero in the range $0<\Delta_{t}<\delta_{c r}{ }^{t}$,

- $\quad$ and $\mathrm{T}_{\mathrm{t}}$ is less than zero in the range $-\delta_{\mathrm{cr}}{ }^{\mathrm{t}}<\Delta_{\mathrm{t}}<0$.

Two possible forms of the function are given as

$$
\begin{aligned}
\phi(\Delta)= & \frac{27}{4} \sigma_{\max } \delta\left(\frac{1}{2}\left(\frac{\Delta_{n}}{\delta}\right)^{2}\left[1-\frac{4}{3}\left(\frac{\Delta_{n}}{\delta}\right)+\frac{1}{2}\left(\frac{\Delta_{n}}{\delta}\right)^{2}\right]\right. \\
& +\frac{1}{2} \alpha\left(\frac{\Delta_{t}}{\delta}\right)^{2}\left[1-2\left(\frac{\Delta_{n}}{\delta}\right)+\left(\frac{\Delta_{n}}{\delta}\right)^{2}\right] \\
& \left.+\frac{1}{2} \alpha\left(\frac{\Delta_{b}}{\delta}\right)^{2}\left[1-2\left(\frac{\Delta_{n}}{\delta}\right)+\left(\frac{\Delta_{n}}{\delta}\right)^{2}\right]\right) \\
\phi(\Delta)=\phi_{n} & -\phi_{n} e^{-\frac{\Delta_{n}}{\delta_{n}}}\left\{\left[1-r+\frac{\Delta_{n}}{\delta_{n}}\right] \frac{1-q}{1-r}-\left[q+\left(\frac{r-q}{r-1}\right) \frac{\Delta_{n}}{\delta_{n}}\right] e^{-\frac{\Delta_{t}^{2}}{\delta_{t}^{2}}}\right\},
\end{aligned}
$$

and

$$
\begin{gathered}
q=\frac{\phi_{t}}{\phi_{n}} \\
r=\frac{\Delta_{n}^{*}}{\delta_{n}}
\end{gathered}
$$

where $\phi_{n}$ and $\phi_{t}$ are the work of normal and tangential separation respectively, $\Delta_{n}{ }^{*}$ is the value of $\Delta_{n}$ after complete shear with $T_{n}=0$ [Needleman, 1987], [Xu and Needleman, 1993]. The work of normal and tangential separation can be explicitly written as 


$$
\phi_{n}=e \sigma_{\max } \delta_{n}, \quad \phi_{t}=\sqrt{\frac{e}{2}} \tau_{\max } \delta_{t},
$$

where $\sigma_{\max }$ and $\tau_{\max }$ are the cohesive surface normal and tangential strengths and $e$ is defined such that the natural logarithm of $e$ equals 1 . Then, for suitably chosen parameters, arbitrary dynamic fracture occurs as a natural outcome of the solution to the boundary value problem.

This method has proven effective in a number of different applications. Siegmund et al have used the technique to model the dynamic propagation of a crack across an interface (e.g. a crack originating in an iron carbide particle propagating into the surrounding ferrite), [Siegmund et al, 1997]. Camacho and Ortiz have incorporated the interfacial cohesion model with a damage model to model impact damage of brittle materials, [Camacho and Ortiz, 1996]. Xu et al have investigated the effects of toughness inhomogeneities on dynamic crack growth, [Xu et al, 1997]. Difficulty arises in applying the method to dimensions typical of engineering structures. The applications have typically been on the order of $10 \mathrm{~mm}$ or less with much smaller elemental dimensions. Typical engineering structures can have dimensions that are many times larger than the structures that have been analyzed thus far with this method.

\subsection{Discrete Element Method}

Potapov et al have developed a cohesive interface method by which rigid or deformable solids are bonded together, [Potapov, Hopkins, and Campbell, 1995a], [Potapov and Campbell, 1996a], [Potapov and Campbell, 1996b]. This bond is 
allowed to break at a predetermined maximum tensile stress and new crack surface is formed, thereby simulating discrete fracture.

In their work, the authors have used three different types of solids, but in all cases, the interface model is the same. One of the models developed employed a rigid 2 dimensional element in which the structural properties and behavior are determined by the interfacial behavior. A second model incorporated a deformable 2 dimensional solid analyzed by the finite element method. In this second model, the structural behavior is determined by a combination of the interface properties and the solid element properties. The third method was an extension of the first method into 3 dimensions. This incorporated 3 dimensional, non-deformable, solids bonded together by a deformable cohesive interface. As with the first model, the structural behavior is completely determined by the properties of the element interfaces.

In each case, the portion of the model that is of interest, with regard to discrete fracture modeling, is the deformable cohesive interface. The interface is modeled as a continuous distribution of "resistive" material. The material can be modeled any of several different ways; elastic, elastic-plastic, viscous, and others. As the structural body deforms, resistive normal and tangential tractions are created at the solid interface. These bond tractions are proportional to normal and tangential stiffnesses, $K_{n}$ and $K_{t}$ respectively. If the normal traction at any point along the interface exceeds the predefined limit stress, $\sigma_{\text {tens }}$, the normal component of traction vanishes at that location. This predefined limit stress is assumed to be representative of the material being modeled. Where appropriate, the tangential displacements are allowed to deform plastically by introducing a Coulomb yield criterion 


$$
\left|\sigma_{t}\right| \leq \operatorname{Coh}+f\left|\sigma_{n}\right|, \quad \sigma_{n} \leq 0
$$

where $\sigma_{t}$ and $\sigma_{n}$ are the average normal and tangential tractions along the entire interface (no compensation is made for portions of the interface that may have failed), Coh is a cohesive limit and $f$ is the internal friction coefficient. A modified Coulomb criterion is used for the case of deformable solid elements

$$
\sqrt{I_{2}} \leq \operatorname{Coh}+f \cdot p
$$

where $I_{2}$ is the second invariant of the stress deviator and $p$ is the pressure as indicated by

$$
I_{2}=\frac{\tau_{i k} \tau_{i k}}{2}
$$

and

$$
p=-\frac{1}{3} \sigma_{u}
$$

The authors stated that this method of introducing plasticity is similar to plastic flow in crystals by the motion of dislocations. They noted that this is only appropriate if the assembly of solid elements is similar in nature to the assembly of crystals in a real material. An assembly of tetrahedra or triangles more closely matches this natural configuration than do brick or quadrilateral solid elements.

In the case of the rigid solid element model, the strain energy in the body is contained solely in the deformable cohesive interface. The fracture energy expended in the creation of new crack surface, $W_{c r}$, is then the work of normal separation from the null state to the critical normal traction state. For the 2 dimensional case the work of separation is given by

$$
W_{c r}=\frac{\sigma_{t e n s}^{2}}{2 K_{n}}
$$


A relationship between the energy released in a breaking interface and the theoretical energy required to create new crack surface was not addressed. In the case of deformable solid elements, the deformable cohesive interface contains only a portion of the total energy in the body. The remainder of the energy is contained within the deformable solid. To relate the energy required to break the interface bond to the theoretical energy required to create new crack surface, the authors have incorporated a criterion by which the interface is modeled as a bond between two infinite plates. The bond is assumed to break at a constant velocity so the energy lost during breakage of the interface can be changed independent of the normal tensile strength of the interface bond.

The authors presented several examples to verify the model. The authors reported that for all simulations, the analysis could be made to model reasonably well the qualitative features of expected results.

The discrete element method has been used to effectively model particulate or granular interactions. If the structure or process being modeled were particulate or granular in nature, the discrete element technique of modeling discrete fracture would likely prove useful. Otherwise, use of the discrete element technique to model discrete fracture in a structural model adds unnecessary complexity to the numerical model. Also, because of the inclusion of rigid or deformable "blocks" with interface deformation zones dispersed throughout the structure, it is not clear that the numerical model would accurately predict dynamic structural behavior. 


\subsection{Element Free Galerkin Method}

Belytschko et al have recently been developing a technique to model fracture using the Element Free Galerkin (EFG) method [Belytschko, Lu, and Gu, 1995], [Belytschko et al, 1995], [Belytschko and Tabbara, 1996], [Sukumar et al, 1997].

The authors state that EFG might be an improvement on other computational methods in fracture because of the ease of creation of new surfaces. A continuum is constructed of a set of nodal points that are contained within the given geometrical description. Moving least square interpolants, in which a list of nearest neighbors must be known, are then used for the trial and test functions used in the Galerkin method. A crack is modeled by introduction of its surface into the geometrical description of the body. Then for nodes on a crack face, the nodes on the opposite crack face are eliminated from the list of nearest neighbors. The method has shown some improvements, and deficiencies, when compared to the finite element method. It has shown some promise in the model of fracture and is still in the development stage.

The method implemented thus far in EFG is not arbitrary in that the crack is defined to grow at a constant velocity perpendicular to the maximum hoop stress, $\sigma_{h}$, as given by

$$
\sigma_{h}=\frac{K_{I}}{\sqrt{2 \pi r}} \Sigma_{h}{ }^{l}(\theta, v)+\frac{K_{I I}}{\sqrt{2 \pi r}} \Sigma_{h}{ }^{I I}(\theta, v),
$$

where $K_{I}$ and $K_{I I}$ are the mode I and mode II stress intensity factors, $r$ and $\theta$ are the polar coordinates centered at the crack tip, and $\Sigma_{h}^{I}$ and $\Sigma_{h}{ }^{I I}$ represent the angular variation of stress as a function of velocity, $v$. The critical value of the maximum hoop stress, $\sigma_{h}^{c}$, is defined by the mode I condition: 


$$
{\sigma_{h}}^{c}=\frac{K_{I}{ }^{c}}{\sqrt{2 \pi r}}
$$

The fracture criterion is then $\sigma_{h} \geq \sigma_{h}^{c}$. Once this criterion is met, the crack propagates through the nodal points in a direction perpendicular to the direction of maximum hoop stress and at a constant velocity.

Although this method is still in relative infancy, the results presented by the authors for both static and dynamic fracture modeling are shown to agree well with known solutions. Some problems are also pointed out and new methods are being developed to address these problems. Enriched methods [Fleming et al, 1997] have been developed by which a singularity corresponding to elastostatic fracture has been incorporated into the formulation. Also, EFG has been coupled with FEM [Belytschko, Organ, and Krongauz, 1995] so that the beneficial aspects of EFG can be incorporated at specific locations in the structure while finite elements can be used elsewhere to take advantage of their ease of use in structural modeling.

\subsection{Molecular Dynamics}

Abraham et al have developed a molecular dynamics model of discrete dynamic fracture in which the forces and motions of atoms in a single crystal are modeled [Abraham et al, 1997], [Abraham et al, 1997], [Abraham, 1997], [Abraham, 1996], [Abraham et al, 1994].

In their analysis, the authors have modeled a single crystal rare-gas solid (e.g. xenon, krypton, and argon) in both two and three dimensions. These single crystal models have dimensions on the order of microns and contain $\sim 10^{6}$ atoms for a twodimensional plate and $\sim 10^{8}$ atoms for the three-dimensional cube. 
Abraham describes molecular dynamics as a simulation technique "based on the motion of a given number of atoms, governed by their mutual interactions described by continuous interatomic potentials" [Abraham, 1997]. In the analysis, the atoms are modeled as particles with mass $m$, each with the necessary degrees of freedom to describe motion in the model space (2-D or 3-D). Each particle obeys classical mechanics defined by a Hamiltonian $H$, as given by

$$
H(r, p)=\kappa(p)+\Phi(r),
$$

where $r=r_{1}, \ldots, r_{n}$ are the atomic coordinates, $p=p_{1}, \ldots, p_{n}$ are the atomic momentum, $\kappa$ is the kinetic energy, and $\Phi$ is the potential energy. The dynamic equations are then

$$
\mathrm{d} r_{i} / \mathrm{d} t=\partial H / \partial p_{i}=p_{i} / m
$$

and

$$
\mathrm{d} p_{i} / \mathrm{d} t=-\partial H / \partial r_{i}=-\partial \Phi(r) / \partial r_{i} .
$$

This simple model of atomic interactions is used to model the dynamic fracture in a plate or cube with an initial central crack subject to symmetric strain rate boundary conditions (Mode I type loading). There are no intrinsic fracture criteria in the model. The atomic bonds break to create new surface when the interatomic separation causes the potential to vanish. The energy expended by creation of new surface is then the energy required to break the atomic bonds. The authors focused particular attention on trying to understand the discrepancy between the theoretical limiting crack speed (Rayleigh wave speed, $C_{r}$ ) and the experimentally observed maximum crack speeds ( $40 \%-60 \%$ of the Rayleigh wave speed).

Significant work has been performed using molecular dynamics analysis on a range of shapes and materials including a graphite sheet, [Omeltchenko et al, 1997], Silicon Diselenide nanowires [Li, Kalia, and Vashista, 1996], amorphous Silicon 
Nitride films [Nakano, Kalia, and Vashista, 1995] and [Vashista et al, 1996] and nanophase Silicon Nitride [Kalia et al, 1997].

The potentials used to model the interaction between the atoms includes one or more of the following; reactive bond order potentials for hydrocarbons, steric repulsion, the effect of charge transfer via Coulomb interaction, the electronic porlarizability of anions through the charge dipole interaction and bond bending and stretching. Investigations have been performed for 2 and 3 dimension specimens. The physical dimensions of the specimens ranging from 10's to 1000's of Angstroms with the number of particles ranging from $\sim 10^{3}-\sim 10^{6}$ atoms.

The authors have paid particular attention to crack front dynamics and the morphology of crack fronts. Their results have shown good agreement between experiment, theory and numerical simulations.

The molecular dynamics technique is useful in the fact that it is capable of modeling the details of fracture on the micromechanics scale. Because of this, the technique has the potential of very accurately modeling the process of fracture within a material. The difficulty encountered with these simulations is that they are currently very expensive computationally. They have thus far only been applied to micro-scale structures and any attempt to apply the technique to typical engineering structures would be computationally difficult and wasteful.

\subsection{Evaluation}

All of the techniques summarized in this section have been shown to model discrete crack growth, for specific problems, along paths that are not predetermined. Although each of these techniques could likely be applied to a wide range of 
problems, difficulty arises when the techniques are applied to engineering scale problems. The methods become either very computationally expensive or simply cumbersome to apply. With the exception of the DEM and EFG methods, the techniques have not been applied to structures of engineering scale at all.

One of the primary goals of this thesis is to provide a useful numerical technique for modeling discrete fracture on engineering scale problems. In the next section of this thesis, the approach to implementing a discrete fracture model in the implicit integration FEM code ABAQUS/Standard is presented followed by the detailed theoretical development. This method is only marginally more complex than a comparable two-dimensional dynamic structural FEM analysis. The work presented herein is a marked improvement over any of the previous attempts at modeling discrete fracture on an engineering scale. 


\section{Approach}

Presented in this section is the approach to modeling a two-dimensional dynamic engineering problem in which the material has a distribution of preexisting macro-sized cracks. The model is developed in a subsequent section using the finite element method with the distributed cracks represented by including a small crack at every interface between two, two-dimensional, solid elements.

To accommodate the geometrical changes that occur during fracture, each 2-D solid element is defined by its own unique nodes. The coincident nodes from adjacent elements are rigidly tied together to form the model. This is shown in Fig. 3.1. A more detailed explanation of the definition of the elements is given in Section 3.1.

The interface has no mass or volume and is completely rigid until failure. Hence, it's only contribution to the analysis is the effect of the crack and the structural and dynamic effects are modeled in the 2-D solid elements. Discussed in Section 3.2, discrete fracture is modeled by defining failure criteria for each interface. If these criteria are met, the displacement constraints on the nodes defining the interface are released and new crack surface is created.

The analysis is formulated on the element level and the implicit finite element code ABAQUS/Standard is used to enforce the boundary conditions, assemble the global equations and solve for the nodal variables. In Section 3.3, the approach to global assembly via ABAQUS/Standard is discussed. 


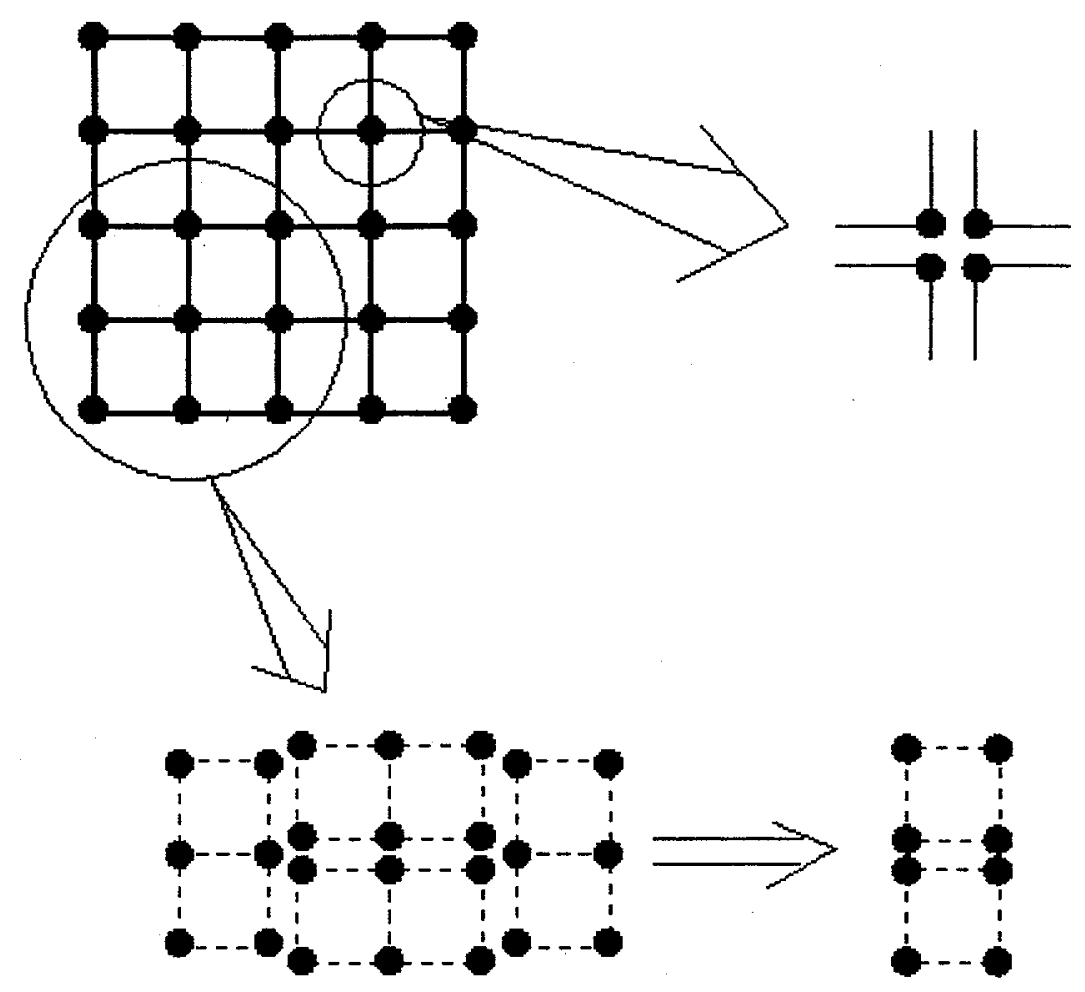

Figure 3.1. Multi-Noding and Overlapping Interface Crack Elements.

\subsection{Defining an Interface Crack Element}

For use in the ABAQUS user element subroutine, an Interface Crack Element (ICE) is defined as two adjacent 2-D solid elements. An exploded view of a simple model of four solid elements is shown in Fig. 3.2. To allow for interface separation, each solid element is defined by unique nodes as shown in the sample model of Fig. 3.2. In the figure, the solid element numbers are boxed, the ICE numbers are circled and the node numbers are plain. The interface between two solid elements is then defined by four node numbers so that the interface can separate during the analysis without redefining solid element connectivity. Each adjacent pair of solid elements that have an interface is a single ICE. Hence, each ICE includes an interface and the 
two adjacent elements that define the interface. In the sample model shown in Fig. 3.2, there are four interfaces, therefore, the model contains four ICEs.
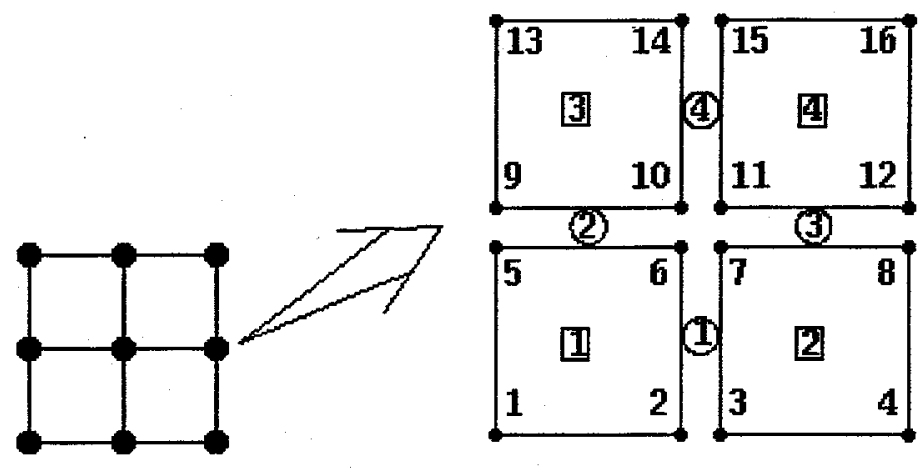

Figure 3.2. Sample model showing an assembly of solid elements and ICEs.

As shown in Fig. 3.3, the ICEs are overlaid to construct the continuum being analyzed. An important feature of this definition is that each interface is defined only once, but each solid element is "defined" multiple times. In the model shown, each solid element is defined twice but it is possible for a solid element to have up to four adjacent solid elements and therefore be defined up to four times.

For each ICE, there are two sets of co-located nodes. These co-located nodes are statically condensed to enforce continuity of displacements across the interface. Then, after the nodal condensation, only one node at each location remains in the analysis until the ICE separates. For an assembly of ICEs, a multi-noded mesh results so that at each node location there are least two nodes and at most four nodes. All of these co-located nodes are statically condensed so that only one node remains in the analysis until failure of one or more of the ICEs occurs. 


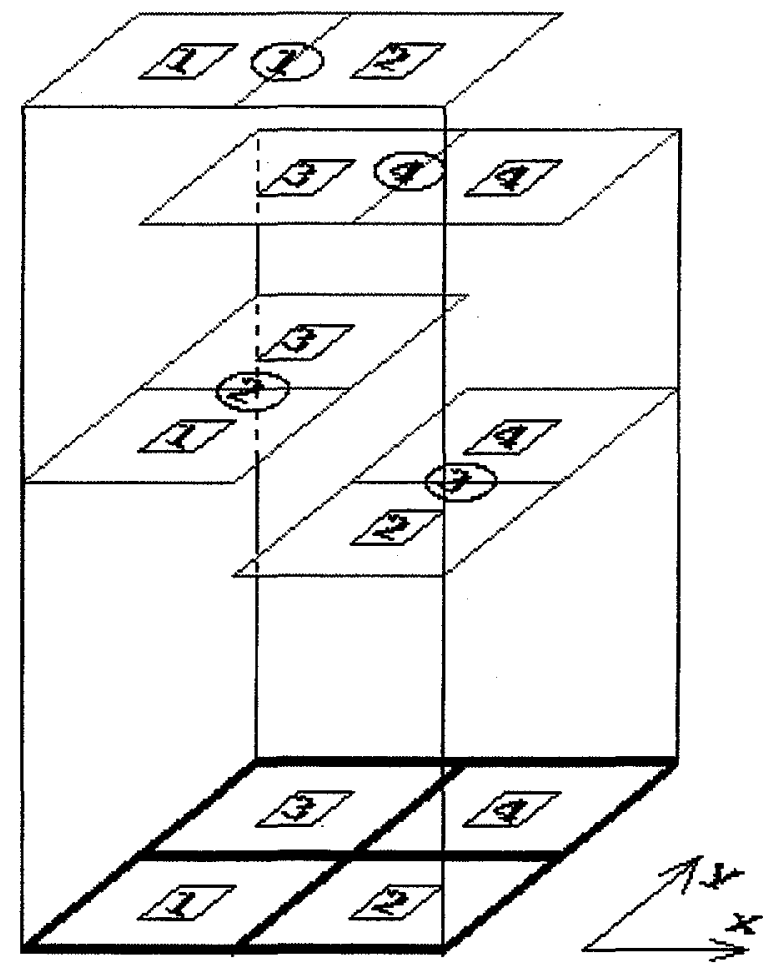

Figure 3.3. Overlaid ICE elements form the model.

The effect of the crack at the interface depends on the stress state at the interface. The average stress state at the interface is assumed to be the average of the states of the two elements that define the interface. The crack in the ICE is assumed to only influence the two elements that make the interface. When the ICE fails, the effect of interface crack on the neighboring elements vanishes.

\subsection{Interface Crack}

At each element interface there is assumed to be a preexisting crack. To model the effects of this crack on the neighboring elements, the crack is modeled as a small crack in an infinite elastic plate that is subject to known far field stresses. Shown in Fig. 3.4 is a crack on the edge of a single solid element with local coordinates $[\xi, \eta]$. The crack is in an infinite elastic plate subject to the far field stresses, $\sigma_{0}, \sigma_{1}$, and $\tau_{0}$. 
The far field stresses are determined from the average stresses in the two neighboring elements at the interface edge. Then, with this model and the known far field stress state, the elastic strain field due to the presence of the crack can be determined.

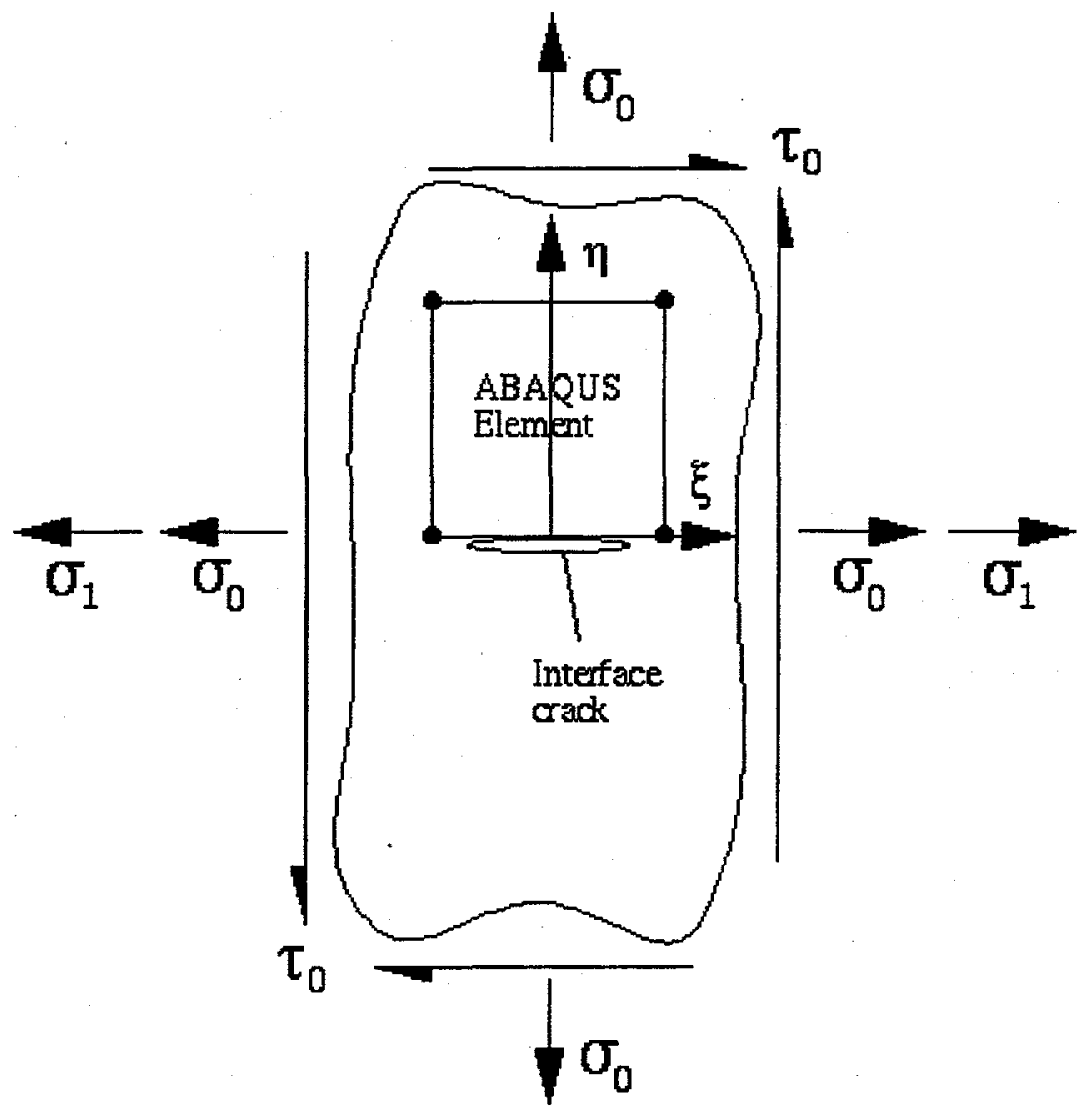

Figure 3.4. Crack in an infinite plate on the edge of a 2-D solid element.

This strain field is treated as an additional external work term in the $\mathrm{Hu}-$ Washizu energy principle. By minimization of this principle, a standard stiffness matrix and load vector are formed for a single element. The stiffness matrix is unaffected by the inclusion of the interface crack, but the load vector includes a term that represents the effects of the crack at the interface. If this term in the load vector is removed (i.e. set the crack size to zero) the standard finite element equations for a 
plane element are recovered. It is possible for a single 2-D solid element to have up to four adjacent elements. Therefore, there could be multiple interfaces on the edges of an element. If there are multiple interfaces, and therefore cracks, on the edge of an element, each crack affects the element assuming that each crack operates independently.

The stress state at the interface also defines failure of the crack based on elastic-plastic fracture mechanics. Crack growth is defined by a crack growth resistance curve which is a function defining the strain energy release rate as a function of crack length. For a brittle material, the curve is a straight line and the material fails when the local strain energy release rate, $G$, exceeds the critical strain energy release rate, $G_{c}$. A rising crack growth resistance curve is typical of ductile materials. The crack growth resistance curve for ductile materials can be represented by the following exponential [Anderson, 1995]

$$
G=\lambda\left(a-a_{o}\right)^{n}+R_{1}
$$

where $R_{1}, \lambda$, and $n$ are material parameters and $G$ is the strain energy release rate at the interface. This equation is inverted to give the change in crack length at each interface based on the local strain energy release rate. Because this criterion only predicts fracture failure, if the initial crack length is very small, the stresses required to grow the crack exceed the failure stress of the material being modeled. To avoid this error, a gross stress failure criterion has been incorporated with the crack growth criteria. The gross stress failure criterion is given by

$$
\sigma_{0}>\sigma_{f}
$$


where $\sigma_{f}$ is the failure stress of the material. If this criterion is met, the displacement constraints are released to create new crack surface.

These failure criteria are solely defined by the material properties and the stress states of the two neighboring elements. Hence, the creation of new crack surface is determined by the solution to the boundary value problem and no a priori assumptions about crack growth speed or direction have been made.

\subsection{Global Assembly and Analysis}

The formulation of the finite element equations from the $\mathrm{Hu}$ - Washizu energy principle are done for a single solid element with one interface crack on its surface. To construct the global continuum and assemble the global equations, the implicit integration finite element code ABAQUS/Standard is used. The implicit formulation using ABAQUS/Standard was chosen for several reasons:

1. Another similar discrete fracture method is currently being developed for use in an explicit finite element code. Comparison of the two methods will provide verification of the implementations.

2. Implicit integration of nodal variables allows for large time steps. Therefore, a wide range of time scales can be modeled.

3. Thermal effects are easily incorporated in the numerical model using ABAQUS analysis methods.

Two, user written, subroutines provided in ABAQUS have been developed to assemble the global problem from the element formulation. The multi - point constraint subroutine (MPC) is used to statically condense the coincident interface nodes until the interface failure criteria have been met. When this occurs, the 
constraint is no longer enforced and the two neighboring elements are uncoupled from each other.

The user element subroutine (UEL) is an ABAQUS subroutine that allows the user to define the behavior of each element. ABAQUS then assembles this elemental information into the global equations and solves for the nodal displacements subject to the specified boundary conditions. The UEL is used to form each element stiffness matrix, the load vector, the mass matrix, and to perform the crack growth analysis for each interface crack. 


\section{Theoretical Development}

\subsection{Hu-Washizu Energy Principle}

The Hu-Washizu energy principle is used as the basis of the numerical formulation. It is a three-field energy principle in which the displacement, strain and stress fields are independently specified. In this section, the fundamental field equations governing an elastic/plastic continuum are shown to result from the variation of the Hu-Washizu principle. It can also be shown in a concise manner that the Hu-Washizu principle can be derived from the weak forms of the field equations of elasticity [Zienkiewicz and Nakazawa, 1984]. Following that of Hu [1955], the fundamental field equations are shown to result as follows:

Let $\Omega$ be a closed region bounded by $\Gamma$, as shown in Fig. 4.1, such that

$$
\Gamma=\Gamma_{u}+\Gamma_{\sigma}
$$

where displacement, $\overline{\mathrm{u}}$, is specified on the boundary $\Gamma_{\mathrm{u}}$, and a traction, $p=\bar{\sigma} \cdot n$, is specified on the boundary $\Gamma_{\sigma}$.

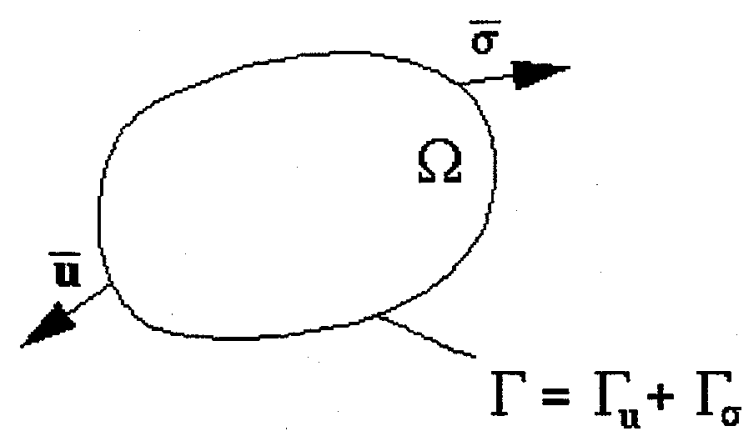

Figure 4.1. Region $\Omega$ bounded by $\Gamma$ with an applied displacement and stress. 
Let $L_{U}$ be the generalized complementary strain energy given by

$$
L_{U}=\sigma_{i j, j} u_{i}+F_{i} u_{i}+e_{i j} \sigma_{i j}-U,
$$

where $\sigma_{i j}$ is the stress tensor, $u_{i}$ is the displacement vector, $F_{i}$ is the body force per unit volume, $e_{i j}$ is the strain tensor, and $U$ is the strain energy density expressed in terms of strain. Then a mechanical energy balance over the region $\Omega$ gives an expression for the total energy as

$$
\Pi_{H W}=\int_{V} L_{U} \mathrm{~d} V-\int_{\Gamma_{u}} \bar{u}_{i} p_{i} \mathrm{~d} \Gamma-\int_{\Gamma_{\sigma}} u_{i}\left(p_{i}-\bar{p}_{i}\right) \mathrm{d} \Gamma,
$$

where the overbar denotes quantities applied to the surface $\Gamma=\Gamma_{u}+\Gamma_{\sigma}$, and

$$
p_{i}=\sigma_{i j} n_{j},
$$

with $n_{j}$ being the unit vector normal to $\Gamma_{\sigma}$.

Then, for stationarity of $\Pi_{H W}$ with respect to the independent variables $u_{i}, e_{i j}$, and $\sigma_{i j}$,

$$
\delta \Pi_{H W}=\delta\left(\int_{\Omega} L_{U} \mathrm{~d} \Omega-\int_{\Gamma_{u}} \bar{u}_{i} p_{i} \mathrm{~d} \Gamma-\int_{\Gamma_{\sigma}} u_{i}\left(p_{i}-\bar{p}_{i}\right) \mathrm{d} \Gamma\right)=0 .
$$

By substitution of the expression for $L_{U}$, eqn. (4.2), into the variation, eqn. (4.5)

$$
\begin{array}{r}
\int_{\Omega}\left(\sigma_{i j, j}+F_{i}\right) \delta u_{i} \mathrm{~d} \Omega-\int_{\Gamma_{u}}\left(p_{i}-\bar{p}_{i}\right) \delta u_{i} \mathrm{~d} \Gamma+\int_{\Omega}\left(\sigma_{i j}-\frac{\partial U}{\partial e_{i j}}\right) \delta e_{i j} \mathrm{~d} \Omega+\int_{\Omega} u_{i} \delta \sigma_{i j, j} \mathrm{~d} \Omega \\
+\int_{\Omega} u_{i} \delta \sigma_{i j, j} \mathrm{~d} \Omega+\int_{\Omega} e_{i j} \delta \sigma_{i j} \mathrm{~d} \Omega-\int_{\Gamma_{u}} \bar{u}_{i} \delta p_{i} \mathrm{~d} \Gamma-\int_{\Gamma_{o}} u_{i} \delta p_{i} \mathrm{~d} \Gamma=0 .
\end{array}
$$

Then by noting that each of the variations, $\delta u_{i}, \delta e_{i j}$, and $\delta\left(\sigma_{i j}\right)$, are arbitrary ${ }^{1}$ and independent, it is concluded that their respective multipliers must be zero in order that eqn. (4.6) be satisfied. The following equations result

\footnotetext{
${ }^{1}$ Except that $\delta \sigma_{i j}=\delta \sigma_{j i}$
} 


$$
\begin{aligned}
& \sigma_{i j, j}+F_{i}=0, \\
& \sigma_{i j}=\frac{\partial U}{\partial e_{i j}}, \\
& e_{i j}=\frac{1}{2}\left(u_{i, j}+u_{j, i}\right),
\end{aligned}
$$

and the essential and natural boundary conditions are

$$
u_{i}=\bar{u}_{i} \quad \text { on } \Gamma_{u}
$$

and

$$
p_{i}=\bar{p}_{i} \quad \text { on } \Gamma_{\sigma} .
$$

Hence, it has been shown that the variation of the Hu-Washizu energy principle, eqn. (4.5), is equivalent to the fundamental field equations governing an elastic/plastic continuum. Therefore, of all possible stress, strain and displacement fields that satisfy the fundamental field equations, the actual fields are those that satisfy eqn. (4.5). This equation is used as the foundation of the numerical approximation in which $\sigma_{i j}, e_{i j}$, and $u_{i}$ are independently approximated.

\subsection{Finite Element Equations}

A finite element approximation is formulated based on the stationarity $\Pi_{H w}$. Switching now to the matrix notation of Weissman and Taylor [1992], the HuWashizu principle, eqns. (4.2) and (4.3), can be restated as

$$
\Pi_{H W}(\mathbf{u}, \sigma, \varepsilon)=\int_{\Omega}\left[\frac{1}{2} \varepsilon^{\mathrm{T}} \mathbf{D} \varepsilon-\varepsilon^{\mathrm{T}} \mathbf{D} \varepsilon_{0}+\varepsilon^{\mathrm{T}} \sigma_{0}+\sigma^{\mathrm{T}}(\mathbf{L u}-\varepsilon)\right] \mathrm{d} \Omega-\Pi_{\mathrm{EXT}},
$$

where $\mathbf{D}$ is the elastic moduli coefficients matrix, $\varepsilon_{0}$ and $\sigma_{0}$ are the initial strain and 
stress tensors respectively, $\Pi_{\mathrm{EXT}}$ is the external work ${ }^{2}$, and $\mathbf{L}$ is the strain displacement operator. For a two dimensional body

$$
\mathbf{L}=\left[\begin{array}{cc}
\frac{\partial}{\partial x} & 0 \\
0 & \frac{\partial}{\partial y} \\
\frac{\partial}{\partial y} & \frac{\partial}{\partial x}
\end{array}\right] .
$$

Then, for stationarity

$$
\int_{\Omega}\left[\delta \sigma^{\mathrm{T}}\left(\mathbf{L} \mathbf{u}-\varepsilon-\varepsilon^{\mathrm{a}}\right)+\delta^{\mathrm{T}}\left(\mathbf{D} \boldsymbol{\varepsilon}-\mathbf{D} \varepsilon_{0}+\sigma_{0}-\sigma\right)+\delta \mathbf{u}^{\mathrm{T}}\left(\mathbf{L}^{\mathrm{T}} \sigma-\mathbf{b}\right)\right] \mathrm{d} \Omega-\int_{\Gamma_{\sigma}} \delta \mathbf{u}^{\mathrm{T}} \mathbf{t}^{\mathrm{a}}=0,
$$

where $\mathbf{b}$ is the body force vector, $\mathbf{t}^{\mathrm{a}}$ are the applied traction boundary conditions, $\varepsilon^{\mathrm{a}}$ are the external strains applied in $\Omega$.

Let the region be subdivided into a finite number of regions (elements) and each region is defined by a finite number of points (nodes). Then the approximations for $\sigma, \mathbf{u}$, and $\varepsilon$ along with their respective variations are given as

$$
\begin{array}{ll}
\sigma=\mathrm{Ss}, & \delta \sigma=\mathbf{S} \delta \mathbf{s}, \\
\varepsilon=\mathrm{Ee}, & \delta \varepsilon=\mathbf{E} \delta \mathrm{e}, \\
\mathbf{u}=\mathbf{N d}, & \delta \mathbf{u}=\mathbf{N} \delta \mathrm{d},
\end{array}
$$

and

where $\mathbf{S}$ and $\mathbf{E}$ are the stress and strain interpolation functions, $\mathbf{s}$ and $\mathbf{e}$ are independent nodal point parameters, $\mathbf{N}$ are the shape functions defined for the subdomain, $\mathbf{d}$ is the vector of displacements of the points defining the subdomain. Substituting eqns.

\footnotetext{
${ }^{2}$ In Weissman and Taylor [1992], the authors give the external work as $\Pi_{\mathrm{EXT}}(\mathbf{u})$. Here it is stated as a general expression of external work, $\Pi_{\mathrm{EXT}}$, to facilitate use in the present work. In Section 4.3.2, the external work is expressed as a function of an externally applied strain field.
} 
(4.15) - (4.17) into eqn. (4.14) and noting that the variations $\delta s, \delta e$, and $\delta \mathbf{d}$ are arbitrary, the following three equations result,

$$
\begin{gathered}
\int_{\Omega_{i}}\left(\mathbf{E}^{\mathrm{T}} \mathbf{D E} \mathbf{E}-\mathbf{E}^{\mathrm{T}} \mathbf{D} \varepsilon_{0}+\mathbf{E}^{\mathrm{T}} \sigma_{0}-\mathbf{E}^{\mathrm{T}} \mathbf{S} \mathbf{s}\right) \mathrm{d} \Omega=0, \\
\int_{\Omega_{i}}\left(\mathbf{S}^{\mathrm{T}} \mathbf{L N d}-\mathbf{S}^{\mathrm{T}} \mathbf{E} \mathbf{e}-\mathbf{S}^{\mathrm{T}} \varepsilon^{\mathrm{a}}\right) \mathrm{d} \Omega=0, \\
\int_{\Omega_{i}}\left(\mathbf{L} \mathbf{N}^{\mathrm{T}} \mathbf{S} \mathbf{s}-\mathbf{N}^{\mathrm{T}} \mathbf{b}\right) \mathrm{d} \Omega-\int_{\Gamma_{i}}\left(\mathbf{N}^{\mathrm{T}} \sigma^{\mathrm{a}}\right) \mathrm{d} \Gamma=0 .
\end{gathered}
$$

and

The following are defined,

$$
\begin{aligned}
& \mathbf{B} \equiv \mathbf{L N}, \\
& \mathbf{H} \equiv \int_{\Omega_{i}} \mathbf{E}^{\mathrm{T}} \mathbf{D E} \mathrm{d} \Omega, \\
& \mathbf{A} \equiv \int_{\Omega_{i}} \mathbf{S}^{\mathrm{T}} \mathbf{E} \mathrm{d} \Omega, \\
& \mathbf{G} \equiv \int_{\Omega_{i}} \mathbf{S}^{\mathrm{T}} \mathbf{B} \mathrm{d} \Omega, \\
& \mathbf{Q} \equiv \int_{\Omega_{i}} \mathbf{S}^{\mathrm{T}} \varepsilon^{\mathrm{a}} \mathrm{d} \Omega, \\
& \mathbf{f} \equiv \int_{\Omega_{i}} \mathbf{N}^{\mathrm{T}} \mathbf{b} \mathrm{d} \Omega+\int_{\mathrm{r}_{i}} \mathbf{N}^{\mathrm{T}} \sigma^{\mathrm{a}} \mathrm{d} \Gamma, \\
& \mathbf{f}_{0} \equiv \int_{\Omega_{i}}\left(\mathbf{E}^{\mathrm{T}} \mathbf{D} \varepsilon_{0}-\mathbf{E}^{\mathrm{T}} \sigma_{0}\right) \mathrm{d} \Omega .
\end{aligned}
$$

By substitution of the above definitions, eqns. (4.18) - (4.20) become

$$
\left[\begin{array}{ccc}
\mathbf{H} & -\mathbf{A}^{\mathrm{T}} & 0 \\
-\mathbf{A} & 0 & \mathbf{G} \\
0 & \mathbf{G}^{\mathrm{T}} & 0
\end{array}\right] \cdot\left[\begin{array}{l}
\mathbf{e} \\
\mathbf{s} \\
\mathbf{d}
\end{array}\right]=\left[\begin{array}{l}
\mathbf{f}_{0} \\
\mathbf{Q} \\
\mathbf{f}
\end{array}\right]
$$


By elimination of e and $\mathbf{s}$;

$$
\mathbf{G}^{\mathrm{T}}\left[\mathbf{A} \mathbf{H}^{-1} \mathbf{A}^{\mathrm{T}}\right]^{-1} \mathbf{G d}=\mathbf{f}+\mathbf{G}^{\mathrm{T}}\left[\mathbf{A} \mathbf{H}^{-1} \mathbf{A}^{\mathrm{T}}\right]^{-1} \mathbf{A} \mathbf{H}^{-1} \mathbf{f}_{\mathbf{0}}+\mathbf{G}^{\mathrm{T}}\left[\mathbf{A} \mathbf{H}^{-1} \mathbf{A}^{\mathrm{T}}\right]^{-1} \mathbf{Q}
$$

If $\mathbf{A}$ is invertable ${ }^{3}$

$$
\mathbf{G}^{\mathrm{T}} \mathbf{A}^{-\mathrm{T}} \mathbf{H} \mathbf{A}^{-1} \mathbf{G d}=\mathbf{f}+\mathbf{G}^{\mathrm{T}} \mathbf{A}^{-\mathrm{T}} \mathbf{f}_{0}+\mathbf{G}^{\mathrm{T}} \mathbf{A}^{-\mathrm{T}} \mathbf{H} \mathbf{A}^{-1} \mathbf{Q}
$$

where superscript $-\mathrm{T}$ denotes the transpose of the inverse. More concisely;

$$
\mathbf{K d}=\mathbf{F},
$$

where $\mathbf{K}$ and $\mathbf{F}$ are the element stiffness matrix and load vector

and

$$
\begin{gathered}
\mathbf{K}=\mathbf{G}^{\mathrm{T}} \mathbf{A}^{-\mathrm{T}} \mathbf{H} \mathbf{A}^{-1} \mathbf{G} \\
\mathbf{F}=\mathbf{f}+\mathbf{G}^{\mathrm{T}} \mathbf{A}^{-\mathrm{T}} \mathbf{f}_{0}+\mathbf{G}^{\mathrm{T}} \mathbf{A}^{-\mathrm{T}} \mathbf{H} \mathbf{A}^{-1} \mathbf{Q}
\end{gathered}
$$

\subsection{Implementation of a 4 Node Plane Element}

The body of interest is taken to be either a thin planar body such that plane stress conditions prevail or a thick restrained body such that plane strain conditions prevail. Between two adjacent solid elements, there is an interface. This interface, which has zero area and is rigid until failure (i.e. allows no normal or tangential separation), contains a crack that produces a strain field in the vicinity of the interface. The vicinity of the interface is taken as the two neighboring elements.

\subsubsection{Stiffness Matrix}

The region, $\Omega$, is discretized into elements as shown in Fig. 4.2. For each 2 dimensional element, the volume, $\Omega_{i}$, is the area of the element multiplied by the thickness, and the surface area, $\Gamma_{i}$, is the arc defining the edges of the element. The

\footnotetext{
${ }^{3}$ See [Weissman and Taylor, 1992] for conditions on the invertability of $\mathbf{A}$. In this work, the conditions are satisfied and $\mathbf{A}$ is invertable.
} 
element is transformed from its global curvilinear coordinates, $[x, y]$, into local linear coordinates, $[\xi, \eta]$, where $-1 \leq \xi \leq 1$, and $-1 \leq \eta \leq 1$.

The interpolation functions in eqns. (4.15) - (4.17) for a 4 node plane element are chosen to be the following linear shape functions [Bennett, 1997]

$$
\begin{gathered}
\mathbf{N}=\left[\begin{array}{cccccccc}
N_{1} & 0 & N_{2} & 0 & N_{3} & 0 & N_{4} & 0 \\
0 & N_{1} & 0 & N_{2} & 0 & N_{3} & 0 & N_{4}
\end{array}\right] \\
\mathbf{S}=\mathbf{E}=\left[\begin{array}{llllllllll}
1 & \xi & \eta & 0 & 0 & 0 & 0 & 0 & 0 \\
0 & 0 & 0 & 1 & \xi & \eta & 0 & 0 & 0 \\
0 & 0 & 0 & 0 & 0 & 0 & 1 & \xi & \eta
\end{array}\right],
\end{gathered}
$$

where the nodal shape functions are

$$
N_{I}=\frac{1}{4}\left(1+\xi_{I} \xi\right)\left(1+\eta_{I} \eta\right)
$$

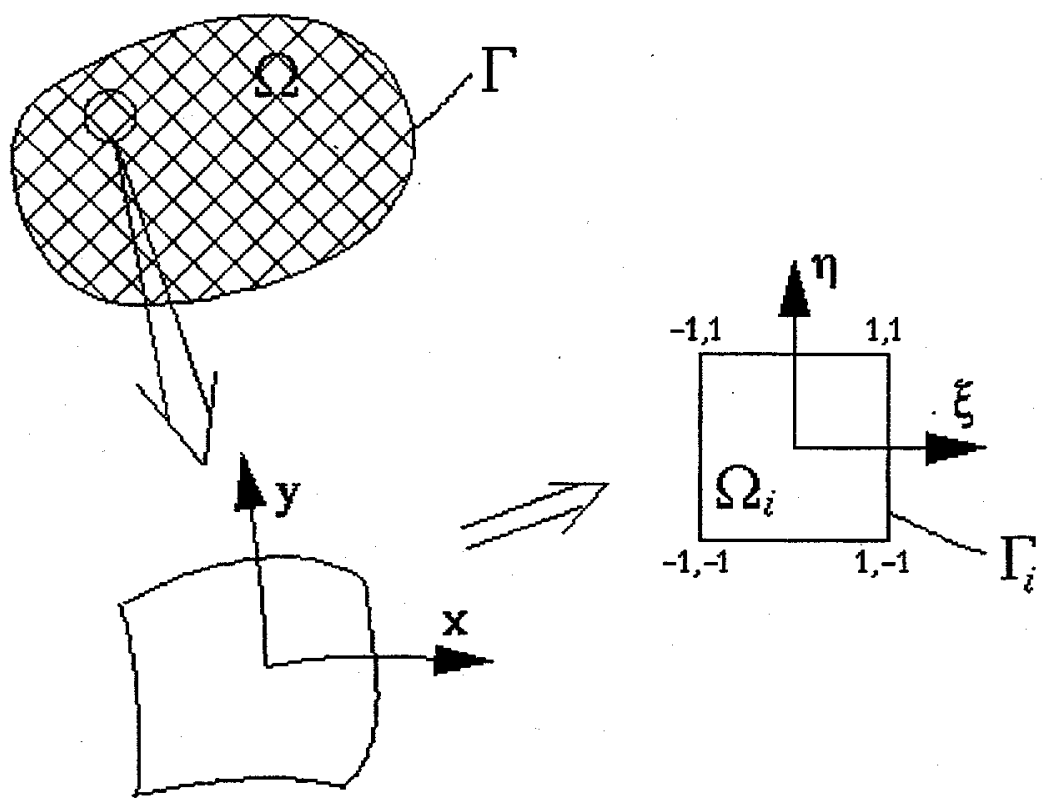

Figure 4.2. Transformation from global $[\mathrm{x}, \mathrm{y}]$ coordinates to local $[\xi, \eta]$ coordinates. 
The global coordinates are given by

$$
\left\{\begin{array}{l}
x \\
y
\end{array}\right\}=\left[\begin{array}{cccccccc}
N_{1} & 0 & N_{2} & 0 & N_{3} & 0 & N_{4} & 0 \\
0 & N_{1} & 0 & N_{2} & 0 & N_{3} & 0 & N_{4}
\end{array}\right] \cdot\left\{\begin{array}{l}
x_{1} \\
y_{1} \\
x_{2} \\
y_{2} \\
x_{3} \\
y_{3} \\
x_{4} \\
y_{4}
\end{array}\right\} .
$$

Defining the Jacobian transformation matrix,

$$
\mathbf{J} \equiv\left[\begin{array}{ll}
\frac{\partial x}{\partial \xi} & \frac{\partial x}{\partial \eta} \\
\frac{\partial y}{\partial \xi} & \frac{\partial y}{\partial \eta}
\end{array}\right]
$$

results in

$$
\left\{\begin{array}{c}
\frac{\partial N_{I}}{\partial \xi} \\
\frac{\partial N_{I}}{\partial \eta}
\end{array}\right\}=\mathbf{J} \cdot\left\{\begin{array}{c}
\frac{\partial N_{I}}{\partial x} \\
\frac{\partial N_{I}}{\partial y}
\end{array}\right\} \text {, and }\left\{\begin{array}{c}
\frac{\partial N_{I}}{\partial x} \\
\frac{\partial N_{I}}{\partial y}
\end{array}\right\}=\mathbf{J}^{-1} \cdot\left\{\begin{array}{c}
\frac{\partial N_{I}}{\partial \xi} \\
\frac{\partial N_{I}}{\partial \eta}
\end{array}\right\}
$$

Then $\mathbf{B}$ from eqn. (4.21) is given by

$$
\mathbf{B}=\left[\begin{array}{cccccccc}
\frac{\partial N_{1}}{\partial x} & 0 & \frac{\partial N_{2}}{\partial x} & 0 & \frac{\partial N_{3}}{\partial x} & 0 & \frac{\partial N_{4}}{\partial x} & 0 \\
0 & \frac{\partial N_{1}}{\partial y} & 0 & \frac{\partial N_{2}}{\partial y} & 0 & \frac{\partial N_{3}}{\partial y} & 0 & \frac{\partial N_{4}}{\partial y} \\
\frac{\partial N_{1}}{\partial y} & \frac{\partial N_{1}}{\partial x} & \frac{\partial N_{2}}{\partial y} & \frac{\partial N_{2}}{\partial x} & \frac{\partial N_{3}}{\partial y} & \frac{\partial N_{3}}{\partial x} & \frac{\partial N_{4}}{\partial y} & \frac{\partial N_{4}}{\partial x}
\end{array}\right]
$$

The elastic moduli coefficient matrices, $\mathbf{D}$, for plane stress and plane strain respectively are 


$$
\mathbf{D}=\frac{E}{1-v^{2}}\left[\begin{array}{ccc}
1 & v & 0 \\
v & 1 & 0 \\
0 & 0 & \frac{1-v}{2}
\end{array}\right], \quad \mathbf{D}=\frac{E(1-v)}{(1+v)(1-2 v)}\left[\begin{array}{ccc}
1 & \frac{v}{1-v} & 0 \\
\frac{v}{1-v} & 1 & 0 \\
0 & 0 & \frac{1-2 v}{2(1-v)}
\end{array}\right]
$$

where $E$ is Young's modulus and $v$ is Poisson's ratio. The differential volume of the element, $d \Omega_{i}$, in terms of local coordinates is

$$
\mathrm{d} \Omega_{\mathrm{i}}=|\mathrm{J}| t \mathrm{~d} \xi \mathrm{d} \eta
$$

where $|\mathbf{I}|$ is the determinant of the Jacobian matrix and $t$ is the element thickness. For plane stress, the thickness is interpolated as follows

$$
t=\left\{\begin{array}{llll}
N_{1} & N_{2} & N_{3} & N_{4}
\end{array}\right\} \cdot\left\{\begin{array}{l}
t_{1} \\
t_{2} \\
t_{3} \\
t_{4}
\end{array}\right\}
$$

and $t=1$ for plane strain.

Equations (4.33) - (4.42) were substituted into eqns. (4.22) - (4.24). These equations were integrated symbolically using the computational software Maple V Release 4. Equation (4.23) was inverted and the multiplication was performed to form the element stiffness matrix, eqn. (4.32a).

\subsubsection{Load Vector}

In this analysis, the initial stress, $\sigma_{0}$, initial strain, $\varepsilon_{0}$, and the externally applied stress $\sigma^{\mathrm{a}}$ are zero so that the external work, $\Pi_{\mathrm{EXT}}$, includes contributions from the body force, $\mathbf{b}$, and the externally applied strain field, $\varepsilon^{\mathbf{a}}$. The load vector, $\mathbf{F}$, from eqn. (4.32b) then reduces to 


$$
\mathbf{F}=\mathbf{f}+\mathbf{G}^{\mathrm{T}} \mathbf{A}^{-\mathrm{T}} \mathbf{H} \mathbf{A}^{-1} \mathbf{Q} .
$$

Where $f$ from eqn. (4.26) is reduced to

$$
\mathbf{f}=\int_{\Omega_{i}} \mathbf{N}^{\mathrm{T}} \mathbf{b} \mathrm{d} \Omega
$$

and the body force vector is given by

$$
\mathbf{b}=\left\{b_{\mathrm{x}} b_{\mathrm{y}}\right\} .
$$

The first term in eqn. (4.43) is integrated symbolically in Maple V. The first four coefficients of the second term in eqn. (4.43) have been assembled for the stiffness matrix and are reused for the load vector. The externally applied strain, $\varepsilon^{\mathrm{a}}$, that appears in $\mathbf{Q}$ (eqn. (4.25)) is the strain field in the element due to an embedded crack located on the edge of the element. Figure 4.3 shows an infinite elastic plate with an embedded crack, subject to the far field stresses $\sigma_{0}, \sigma_{1}$, and $\tau_{0}$. The embedded crack is on the edge of a plane element with the origin of the local coordinates $[\xi, \eta]$ located at the center of the crack. The external strain field, $\varepsilon^{a}$, is the strain in the adjacent element due only to the presence of the crack on its edge.

The strain field for a crack in an infinite elastic plate subject to the in-plane far field stresses shown in Fig. 4.3 can be determined by introducing a complex stress function $\mathrm{Z}(z)$, where $z=\xi+i \eta$ and $i=\sqrt{-1}$ [Anderson, 1995],

$$
Z(z)=\frac{z}{\sqrt{z^{2}-a^{2}}}, \quad Z^{\prime}(z)=\frac{-a^{2}}{\left(z^{2}-a^{2}\right)^{\frac{3}{2}}},
$$

where $a$ is the half crack width. 


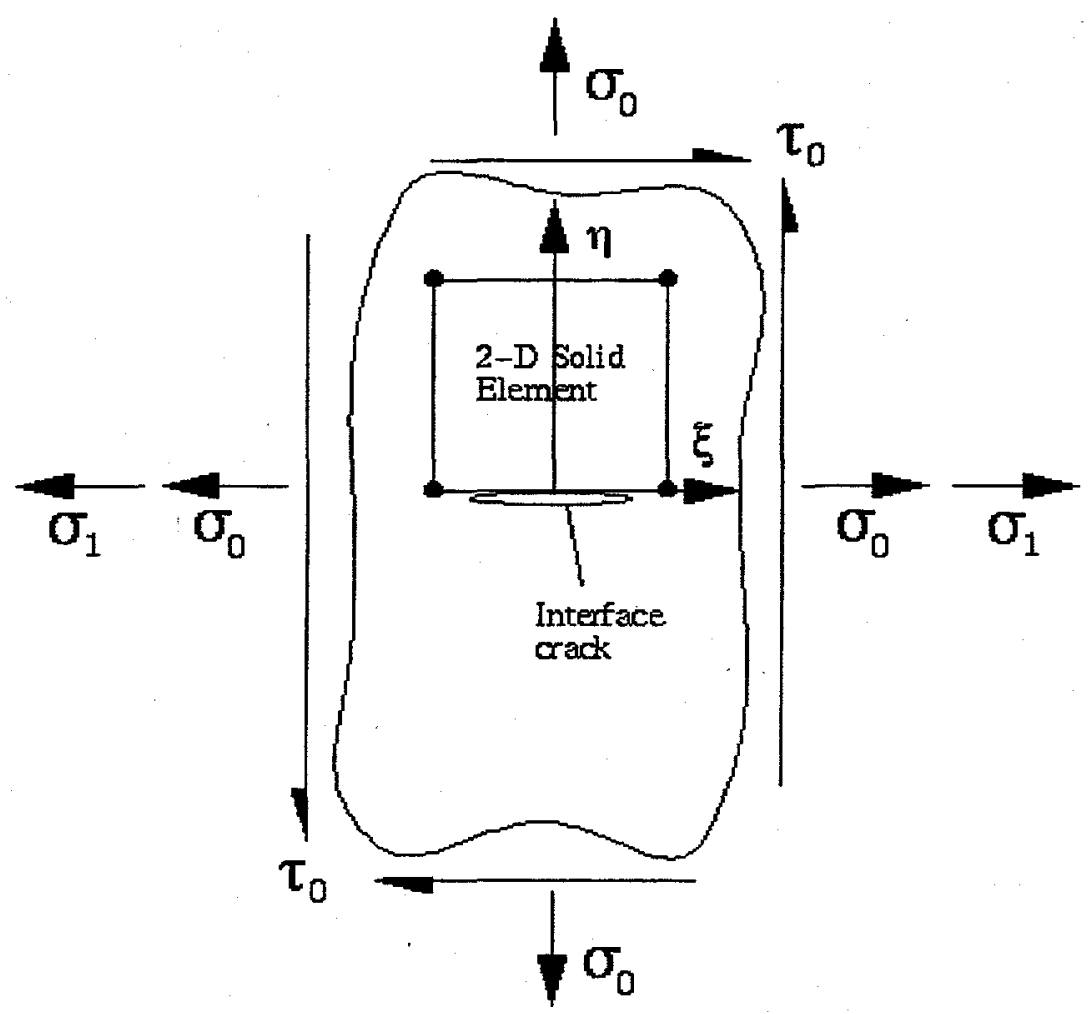

Figure 4.3. Crack in an infinite plate on the edge of a 2-D solid element.

For Mode I loading, $\tau_{0}=0$ and the Mode I stresses are given by

$$
\begin{aligned}
& \sigma_{\xi \xi}=\sigma_{0}\left(\operatorname{Re}(Z)-\eta \operatorname{Im}\left(Z^{\prime}\right)\right)+\sigma_{1}, \\
& \sigma_{\eta \eta}=\sigma_{0}\left(\operatorname{Re}(Z)+\operatorname{Im}\left(Z^{\prime}\right)\right)
\end{aligned}
$$

and

$$
\tau_{\xi \eta}=-\sigma_{0} \eta \operatorname{Re}\left(Z^{\prime}\right)
$$

and

$$
\tau_{\xi \eta}=\tau_{0}\left(\operatorname{Re}(Z)-\eta \operatorname{Im}\left(Z^{\prime}\right)\right)
$$


The stress - strain relations for plane stress and plane strain elastic behavior are given by

$$
\begin{gathered}
\varepsilon_{\xi \xi}=\frac{1}{\mathrm{E}}\left(\sigma_{\xi \xi}-v \sigma_{\eta \eta}\right), \quad \varepsilon_{\eta \eta}=\frac{1}{\mathrm{E}}\left(\sigma_{\eta \eta}-v \sigma_{\xi \xi}\right), \quad \varepsilon_{\xi \eta}=\frac{1}{2 \mathrm{G}} \tau_{\xi \eta} ; \\
\varepsilon_{\xi \xi}=\frac{1+v}{\mathrm{E}}\left((1-v) \sigma_{\xi \xi}-v \sigma_{\eta \eta}\right), \quad \varepsilon_{\eta \eta}=\frac{1+v}{\mathrm{E}}\left((1-v) \sigma_{\eta \eta}-v \sigma_{\xi \xi}\right), \quad \varepsilon_{\xi \eta}=\frac{1}{2 \mathrm{G}} \tau_{\xi \eta},
\end{gathered}
$$

where $G$ is the shear modulus of the material.

Then, by superposition of Mode I and Mode II stresses, eqns. (4.53) and (4.54) become the following

$$
\begin{gathered}
\varepsilon_{\xi \xi}=\frac{1}{\mathrm{E}}\left[\left(\sigma_{0}\left(\operatorname{Re}(Z)-\eta \operatorname{Im}\left(Z^{\prime}\right)\right)+\sigma_{1}+\tau_{0}\left(2 \operatorname{Im}(Z)+\eta \operatorname{Re}\left(Z^{\prime}\right)\right)\right)-\right. \\
\left.v\left(\sigma_{0}\left(\operatorname{Re}(Z)+\eta \operatorname{Im}\left(Z^{\prime}\right)\right)-\tau_{0} \eta \operatorname{Re}\left(Z^{\prime}\right)\right)\right], \\
\varepsilon_{\eta \eta}=\frac{1}{\mathrm{E}}\left[\left(\sigma_{0}\left(\operatorname{Re}(Z)+\eta \operatorname{Im}\left(Z^{\prime}\right)-\tau_{0} \eta \operatorname{Re}\left(Z^{\prime}\right)\right)-\right.\right. \\
\left.v\left(\sigma_{0}\left(\operatorname{Re}(Z)-\eta \operatorname{Im}\left(Z^{\prime}\right)\right)+\sigma_{1}+\tau_{0}\left(2 \operatorname{Im}(Z)+\eta \operatorname{Re}\left(Z^{\prime}\right)\right)\right)\right], \\
\varepsilon_{\xi \eta}=\frac{1}{2 \mathrm{G}}\left[-\sigma_{0} \eta \operatorname{Re}\left(Z^{\prime}\right)+\tau_{0}\left(\operatorname{Re}(Z)-\eta \operatorname{Im}\left(Z^{\prime}\right)\right)\right],
\end{gathered}
$$

for plane stress, and

$$
\begin{gathered}
\varepsilon_{\xi \xi}=\frac{1+v}{\mathrm{E}}\left[(1-v)\left(\sigma_{0}\left(\operatorname{Re}(Z)-\eta \operatorname{Im}\left(Z^{\prime}\right)\right)+\sigma_{1}+\tau_{0}\left(2 \operatorname{Im}(Z)+\eta \operatorname{Re}\left(Z^{\prime}\right)\right)\right)-\right. \\
\left.v\left(\sigma_{0}\left(\operatorname{Re}(Z)+\eta \operatorname{Im}\left(Z^{\prime}\right)\right)-\tau_{0} \eta \operatorname{Re}\left(Z^{\prime}\right)\right)\right], \\
\varepsilon_{\eta \eta}=\frac{1+v}{\mathrm{E}}\left[( 1 - v ) \left(\sigma_{0}\left(\operatorname{Re}(Z)+\eta \operatorname{Im}\left(Z^{\prime}\right)-\tau_{0} \eta \operatorname{Re}\left(Z^{\prime}\right)\right)-\right.\right. \\
\left.v\left(\sigma_{0}\left(\operatorname{Re}(Z)-\eta \operatorname{Im}\left(Z^{\prime}\right)\right)+\sigma_{1}+\tau_{0}\left(2 \operatorname{Im}(Z)+\eta \operatorname{Re}\left(Z^{\prime}\right)\right)\right)\right],
\end{gathered}
$$




$$
\varepsilon_{\xi \eta}=\frac{1}{2 \mathrm{G}}\left[-\sigma_{0} \eta \operatorname{Re}\left(Z^{\prime}\right)+\tau_{0}\left(\operatorname{Re}(Z)-\eta \operatorname{Im}\left(Z^{\prime}\right)\right)\right]
$$

for plane strain.

Far field strains analogous to the far field stress can be defined, i.e. $\varepsilon_{0} \varepsilon_{1} \gamma_{0}$. The strain in the vicinity of the crack, due only to the presence of the crack, $\varepsilon^{\mathrm{a}}$, is then the total strain field around the crack minus the far field strain.

$$
\varepsilon^{a}=\left\{\begin{array}{c}
\varepsilon_{\xi \xi}-\left(\varepsilon_{0}+\varepsilon_{1}\right) \\
\varepsilon_{\eta \eta}-\varepsilon_{0} \\
\varepsilon_{\xi \eta}-\gamma_{0}
\end{array}\right\}
$$

Plotted in the local coordinate system defined by Fig. 4.3, Figs. 4.4 and 4.5 show the three components of the additional strain field of eqn. (4.61) in a plane stress (Fig. 4.4) and plane strain (Fig. 4.5) element. The strain fields shown are for a segment of the local coordinates defined by $0 \leq \xi \leq 1$ and $0 \leq \eta \leq 1$. The strain fields for $-1 \leq \xi \leq 0$ can be determined by noting the symmetries and skew symmetries of the strain components. The edge crack is defined along the line $-0.5 \leq \xi \leq 0.5, \xi=0$. Hence, the crack has a half width, $a$, of 0.5 . The far field stresses are unity. The Young's Modulus of the material is unity and Poisson's ratio is $1 / 3$.

Depending on the model (plane stress or plane strain), the strain fields of Fig. 4.4 or Fig. 4.5 are integrated numerically with Gauss Quadrature. Equation (4.26) is assembled and the multiplication is performed to form the load vector, eqn. (4.43). 

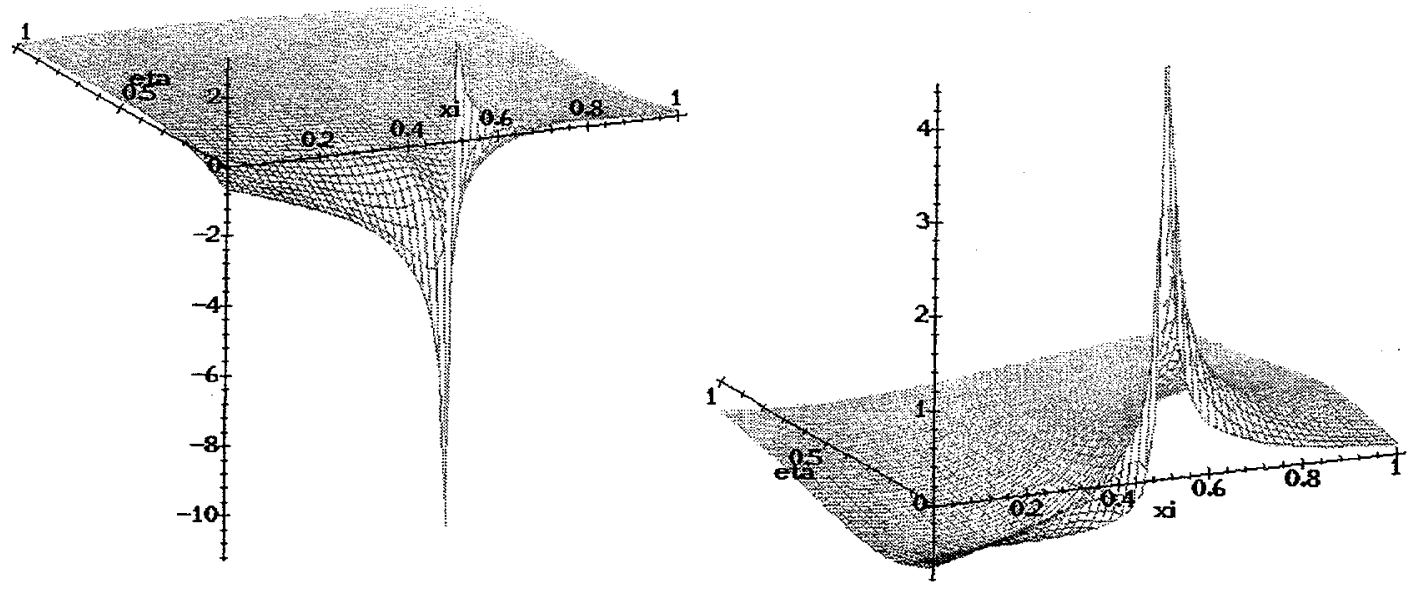

i)

ii)

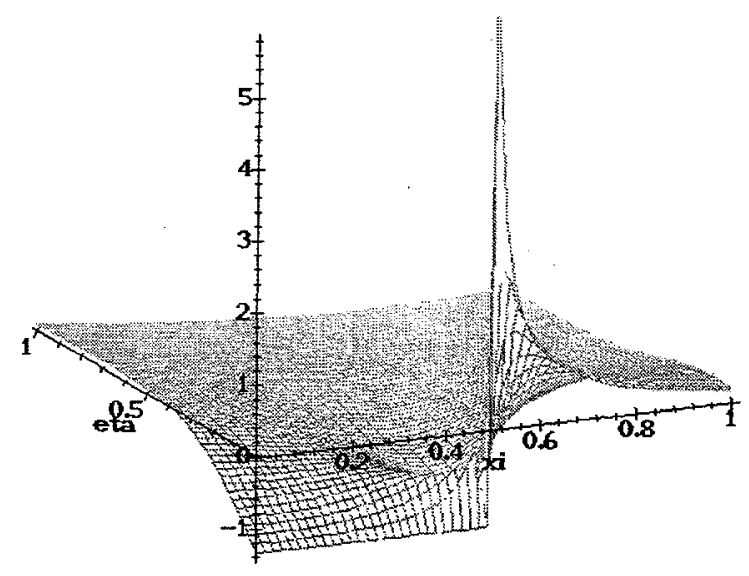

iii)

Figure 4.4. Components of the strain field in a plane stress element due to the presence of a crack at the interface. $\mathrm{E}=\sigma_{0}=\sigma_{1}=\tau_{0}=1, v=1 / 3, a=0.5$ i) $\varepsilon_{1}^{\mathrm{a}}$ ii) $\varepsilon_{2}^{\mathrm{a}}$ iii) $\varepsilon_{3}^{\mathrm{a}}$ 


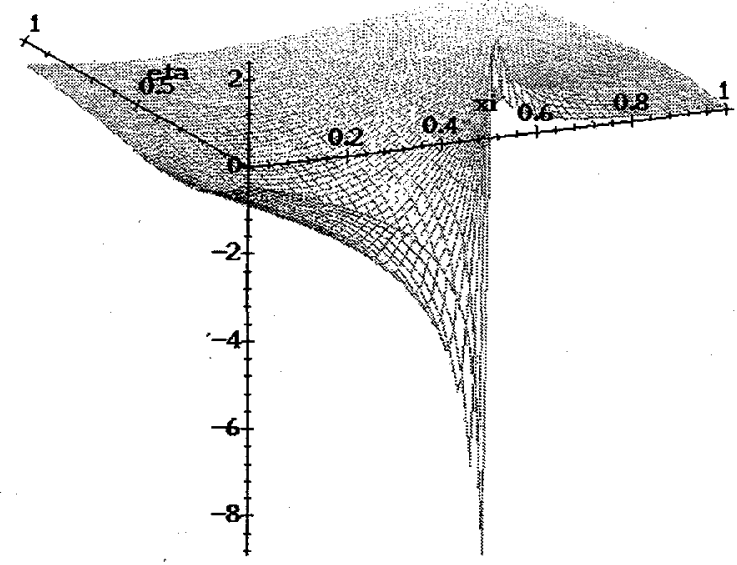

i)

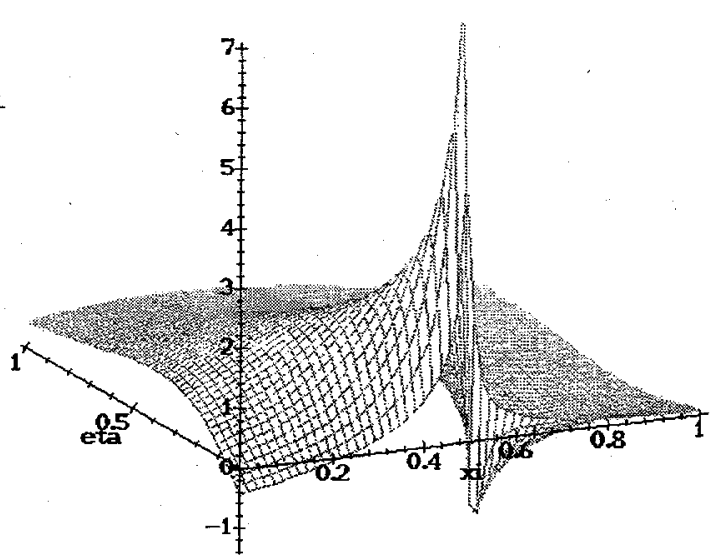

ii)

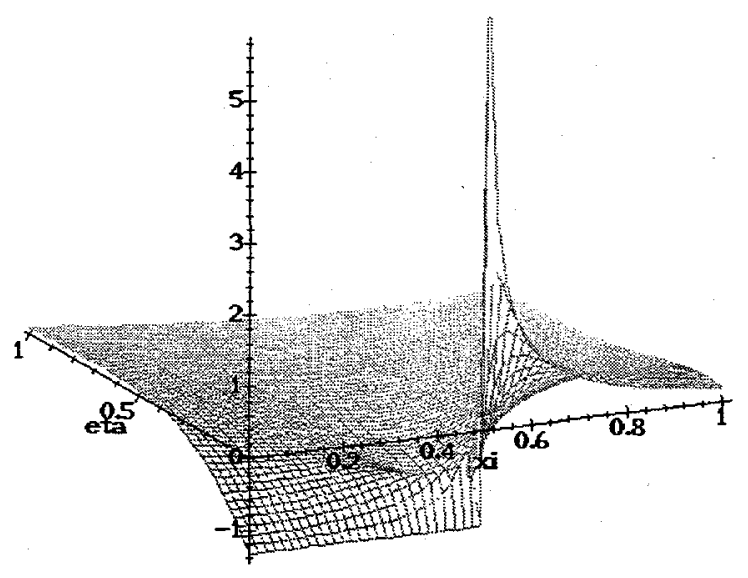

iii)

Figure 4.5. Components of the strain field in a plane strain element due to the presence of a crack at the - interface. $\mathrm{E}=\sigma_{0}=\sigma_{1}=\tau_{0}=1, v=1 / 3, a=0.5$ i) $\varepsilon_{1}^{\mathrm{a}}$ ii) $\varepsilon_{2}^{\mathrm{a}}$ iii) $\varepsilon_{3}^{\mathrm{a}}$ 


\subsection{ABAQUS/Standard Implementation}

\subsubsection{User Element}

ABAQUS/Standard (referred to as ABAQUS) provides a means by which the user can completely define the behavior of a finite element in an external subroutine. This user element subroutine (UEL) is coded to define the contribution of an individual element to the model. For each call to the UEL subroutine, the necessary information (nodal displacements, velocities, accelerations, state variables, etc.) is passed into the subroutine and a Jacobian matrix ${ }^{4}$ and residual vector for the individual element must be calculated and returned. ABAQUS assembles this information to form the complete system of equations and solve for the nodal variables.

In Fig. 4.6, a region is discretized into four solid elements. Shown on the right in Fig. 4.6, an Interface Crack Element (ICE) is defined as two adjacent solid elements with unique node numbers and a crack at the interface. The two sets of co-located nodes at the interface remain coincident until failure. The individual ICEs are then assembled into the region being analyzed.

As described in Section 3.1 and also shown in Fig. 4.6, the definition of a single user element in this analysis can be conceptually thought of as two adjacent 2-D solid elements with a crack at the interface. Because of the definition of the ICE, it is likely that solid elements will be defined multiple times for the assembled model, but each ICE in the model will be defined only once.

\footnotetext{
${ }^{4}$ The Jacobian matrix is defined in ABAQUS/Standard. It is not the same as the Jacobian transformation matrix defined by eqn. (4.37).
} 


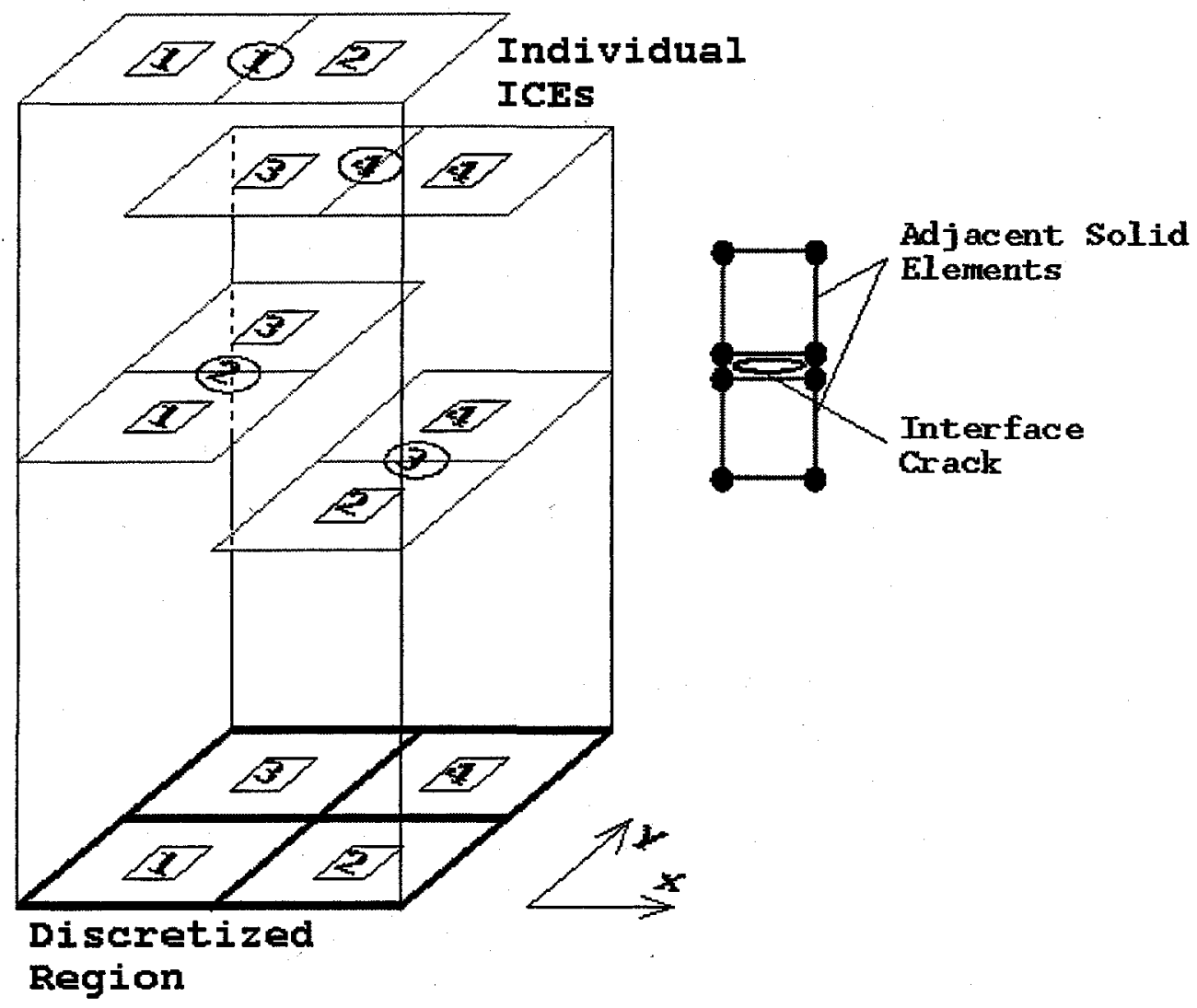

Figure 4.6. Interface Crack Element.

For an assembled matrix for a model, ABAQUS solves the following set of equations by an appropriate iterative method ${ }^{5}$;

$$
\tilde{K}^{N M} c^{M}=R^{M}
$$

and

$$
u^{N}=u^{N}+c^{N}
$$

where $N$ and $M$ represent the system degrees of freedom ( 2 components of displacement for each node in the present analysis), $\widetilde{K}^{N M}$ is the Jacobian matrix, $c^{M}$ is the increment in nodal displacements, $R^{M}$ is the residual vector and $u^{N}$ is the nodal displacement vector. Then for an exact solution, the residual and increment in

\footnotetext{
${ }_{5}^{5}$ ABAQUS/Standard uses a Newton or Quasi-Newton solution method. Refer to the ABAQUS/Standard User's Manual and Theory Manual for descriptions and use of these methods.
} 
displacement would both equal zero. In practice, this is almost never realized and convergence criteria are specified.

For implicit dynamic analysis, the Hilber, Hughes, Taylor integration operator replaces the equilibrium equation with

$$
\left.M^{N M} \ddot{u}^{M}\right|_{t+\Delta t}+(1+\alpha)\left(\left.I^{N}\right|_{t+\Delta t}-\left.P^{N}\right|_{t+\Delta t}\right)-\alpha\left(\left.I^{N}\right|_{t}-\left.P^{N}\right|_{t}\right)+\left.L^{N}\right|_{t+\Delta t}=0
$$

where $\alpha$ is a numeric damping coefficient, $M^{N M}$ is the consistent mass matrix defined by the mass density, $\rho_{0}$, and the vector of interpolation functions, $\mathbf{N}$, as follows;

$$
M^{N M}=\int_{\Omega} \rho_{0} \mathbf{N}^{N} \cdot \mathbf{N}^{M} \mathrm{~d} \Omega
$$

$I^{N}, P^{N}$ are the internal and external force vectors. For the current analysis, the external force vector is zero and the internal force vector is given by

$$
I^{N}=K^{N M} u^{M}+F^{N}
$$

where $K^{N M}$ is the stiffness matrix defined by eqn. (4.32a), $u^{M}$ is the nodal displacement vector and $F^{N}$ is the load vector defined by eqn. (4.43). $L^{N}$ is the vector of Lagrange multiplier forces which is zero in this analysis.

The time domain integration to compute the displacement and velocity is carried out using the Newmark formulae

$$
\begin{gathered}
\left.u\right|_{t+\Delta t}=\left.u\right|_{t}+\left.\Delta t \dot{u}\right|_{t}+\Delta t^{2}\left[\left.\left(\frac{1}{2}-\beta\right) \ddot{u}\right|_{t}+\left.\beta \ddot{u}\right|_{t+\Delta t}\right] \\
\left.\dot{u}\right|_{t+\Delta t}=\left.\dot{u}\right|_{t}+\Delta t\left[\left.\left(\frac{1}{2}-\gamma\right) \ddot{u}\right|_{t}+\left.\gamma \ddot{u}\right|_{t+\Delta t}\right],
\end{gathered}
$$

where

$$
\beta=\frac{1}{4}(1-\alpha)^{2}, \quad \gamma=\frac{1}{2}-\alpha
$$


Solving eqn. (4.67) for $\left.\ddot{u}\right|_{t+\Delta t}$ and substituting into eqn. (4.64) in the absence of interface crack influence yields

$$
\left.\left[\frac{M^{N M}}{\beta \Delta t^{2}}+(1+\alpha) K^{N M}\right] u\right|_{t+\Delta t}-\frac{M^{N M}}{\beta}\left[\frac{\left.u\right|_{t}}{\Delta t^{2}}+\frac{\left.\dot{u}\right|_{t}}{\Delta t}+\left.\left(\frac{1}{2}-\beta\right) \ddot{u}\right|_{t}\right]-\left.\alpha K^{N M} u\right|_{t}=0 .
$$

Equation (4.64) applies to the system as well as each individual element so that the global Jacobian matrix and residual vector can be assembled from the elemental matrices and vectors. The elemental contribution to the Jacobian matrix is then the coefficient on $\left.u\right|_{t+\Delta t}$ in eqn. (4.70),

$$
\tilde{K}^{N M}=\left[\frac{M^{N M}}{\beta \Delta t^{2}}+(1+\alpha) K^{N M}\right]
$$

The elemental contribution to the residual vector is the left-hand side of eqn. (4.64),

$$
R^{N}=-\left.M^{N M} \ddot{u}^{M}\right|_{t+\Delta t}+\left.(1+\alpha) G^{N}\right|_{t+\Delta t}-\left.\alpha G^{N}\right|_{t}
$$

where $G^{N}$ is the combination of internal and external load vectors for the element.

Because of the overlapping definition of a user element stated above and shown in Fig. 4.6, care must be taken so as not to assign multiple stiffness and mass to a single element. To accomplish this, the assignment of the mass matrix and stiffness matrix is recorded during each solution iteration. If these matrices have been assigned to an element on a previous call to the UEL subroutine during a single iteration, then a new mass matrix and stiffness matrix are not defined. Hence, the mass and stiffness for each 2-D solid element are defined only once for each iteration. Because each interface, and therefore, each interface crack, is only defined once, the contributions to the right hand side vector due to the presence of a crack are assigned for each call to 
the UEL subroutine. Each crack on the border of an element will then affect the behavior of the element.

\subsubsection{Multi-Point Constraint}

The MPC subroutine allows the user to constrain some or all of the degrees of freedom of nodes. The use here will be to rigidly "tie" two, 2-D solid, elements together to create the continuous interface and release the "tie" to create new crack surface when the interface failure criteria are met.

As used in this analysis, the degrees of freedom of one node are eliminated by attaching them to the degrees of freedom of the coincident node on the adjacent 2-D solid element. The constraint for the $i^{\text {th }}$ degree of freedom is then given by

$$
f_{i}\left(\mathbf{u}^{1}, \mathbf{u}^{2}\right)=0
$$

where $\mathbf{u}^{1}$ and $\mathbf{u}^{2}$ are the vectors of degrees of freedom of the dependent and independent nodes respectively. To remove the two components of displacement of the dependant node, eqn. (4.73) can be explicitly written as

and

$$
\begin{gathered}
f_{1}\left(\mathbf{u}^{1}, \mathbf{u}^{2}\right)=u_{1}{ }^{1}-u_{1}{ }^{2} \\
f_{2}\left(\mathbf{u}^{1}, \mathbf{u}^{2}\right)=u_{2}{ }^{1}-u_{2}{ }^{2}
\end{gathered}
$$

Due to the incremental nature of the solution procedure, a linearized set of constraints can be used for the calculation of equilibrium;

$$
\delta f_{i}=A_{i}^{1} \cdot \delta \mathbf{u}^{1}+A_{i}^{2} \cdot \delta \mathbf{u}^{2}
$$

The matrix representing the coefficients in the linearized constraint equations is given by

$$
A_{i j}{ }^{I}=\frac{\partial f_{i}}{\partial \mathbf{u}_{j}{ }^{I}}
$$


where $i, j=1,2$ represent the degrees of freedom and $I=1,2$ represents the dependent and independent nodes. Substitution of eqns. $(4.74 a, b)$ into eqn. (4.76) yields

$$
\begin{aligned}
\mathbf{A}^{1} & =\left[\begin{array}{ll}
1 & 0 \\
0 & 1
\end{array}\right], \\
\mathbf{A}^{2} & =\left[\begin{array}{cc}
-1 & 0 \\
0 & -1
\end{array}\right] .
\end{aligned}
$$

It can be noted at this point that $(n-1)$ constraints, where $n$ is the number of nodes at a location, are required to statically condense all nodes at a location into one node. Any greater number of constraints results in either under-constrained or redundancy depending on the order they are evaluated. If all constraints are properly applied, only one node will remain independent, all other nodes at the location are dependent nodes. The under-constrained condition results from a dependent node being attached to a previously dependent node. Redundancy results from the similar configuration except that the sole remaining independent node is attached to a previously dependent node ${ }^{6}$. The former case has a simple solution in that it can be corrected by simply listing the constraints in the proper order. The latter case presents somewhat of a challenge because it can not be determined a priori which node should be the independent node.

For example, Fig. 4.7 shows an assembly of 4 ICEs. At the intersection of the four user elements, there would naturally be four multi-point constraints with only four nodes at the location (i.e. nodes $6,7,10,11$ ). At this point, any node would suffice as the remaining independent node, but this would only be true until one or more of the interface cracks failed. 


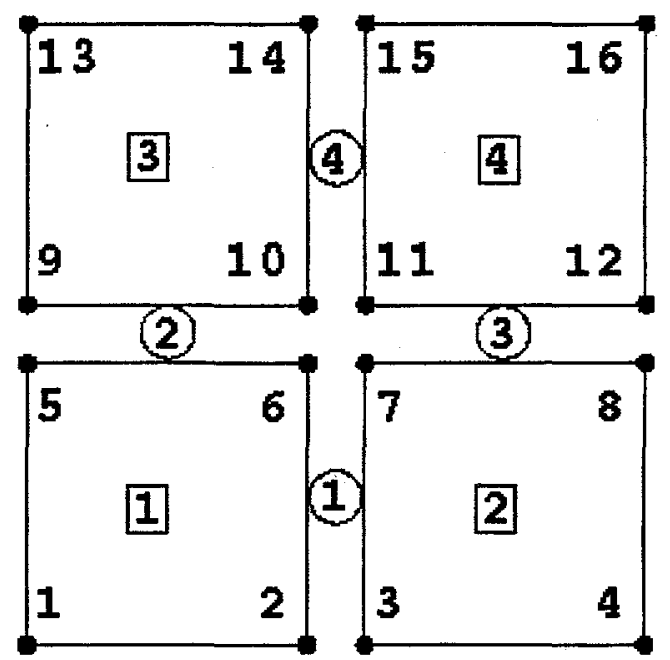

Figure 4.7. A simple system consisting of four 2-D solid elements (boxed numbers), four Interface Crack Elements (the circled numbers) and 16 nodes.

To illustrate this point, suppose steps $\mathrm{a}, \mathrm{b}$ and $\mathrm{c}$ were used as the constraints to tie the four nodes together:

a) node 6 tied to node 7 , eliminating node 6 ;

b) node 7 tied to node 11 , eliminating node 7 ;

c) node 11 tied to node 10 , eliminating node 11 .

Nodes 6,7, and 11 have been eliminated, therefore, the remaining independent node is node 10. If ICE 3 failed, the constraints defining the interface would be released, and elements 2 and 4 would be allowed to separate. The four nodes at the intersection should remain coincident. If constraint $b$ were released, node 7 would also become independent. The system becomes under-constrained because there are two independent nodes at a single location. Hence, the nodal condensation would not be correct.

\footnotetext{
${ }^{6}$ Because the multi-point constraints are evaluated in the order listed in the input, a node that has been a dependent node can no longer be used as an independent node.
} 
To avoid this problem, a system was developed by which the remaining independent node at a location becomes the dependent node when one or more failures occur at that location. The node is attached to the formerly dependent node of one of the failed ICEs at that location. For the example given above, when ICE 3 failed, node 10 would be tied to node 7 so that node 7 is the remaining independent node. This ensures that the multi-point constraints are correctly enforced. The method, presented in detail in Section 1.2.1.2 of the Appendix, has proven to work for any combination of ICE failures.

\subsection{Interface Failure}

The contributions to the Jacobian matrix and residual vector are the two pieces of information required by ABAQUS to define the behavior of the element. If there were no interface separation, those two arrays would be sufficient to define the element. Because of the interface crack, which includes crack growth and interface failure, other calculations must be performed and an indication of element failure must be passed to the multi-point constraint (MPC) subroutine. This section describes the evaluation of the interface failure.

\subsubsection{Convergence}

To determine the growth and failure of the crack at the interface, it should first be pointed out that the solution technique used by ABAQUS is a non-monotonic iterative process. This means that the result of each iteration is a wrong solution until the convergence criteria have been met. Therefore, the interface crack behavior for each user element is not correct until the solution has converged. This presents somewhat of a problem in that if the solution, whether correct or incorrect, dictates 
that the interface has failed, there is a significant change in the problem. It would be desirable to have the solution estimate approach the converged solution from below. In other words, the estimated average stress state at the interface would be lower than the correct average state until a solution has been reached.

One method to ensure that the problem does not undergo significant change (an interface crack failure) due to a poor estimate of the solution is to employ user defined convergence criteria. Until these criteria are met, there are no interface failures. Because of the limited amount of information passed into the UEL subroutine, a somewhat less strict convergence criteria than ABAQUS uses is employed. ABAQUS uses convergence criteria based on the model residual vector, eqn. (4.69), and the ratio of the largest displacement correction, $c^{N}$ from eqn. (4.59), to the largest increment of displacement. The user defined convergence criteria used in the UEL subroutine are defined using the nodal displacements

$$
\frac{c^{N}}{u_{i}^{N}}<\lambda, \quad c^{N}<\delta, \quad u_{i}^{N}<\varepsilon,
$$

where $u_{i}^{N}$ is the vector of increments in nodal point displacements, $\lambda, \delta$, and $\varepsilon$ are user defined convergence criteria. While these criteria are not always a correct predictor of solution convergence, the user can determine when the method is in error. The method of evaluating solution convergence is presented in Section 1.2.2.3 of the Appendix.

\subsubsection{Interface Failure}

After the convergence criteria have been met, a solution to within reasonable tolerances has been reached and the interface failure criteria can be evaluated to determine crack growth and failure at each interface. 
The elastic-plastic crack growth of the crack at the element interface is based on a crack growth resistance curve. A typical curve shown in Fig. 4.8 gives the relation between the crack half width, $a$, and the local strain energy release rate, $G$.

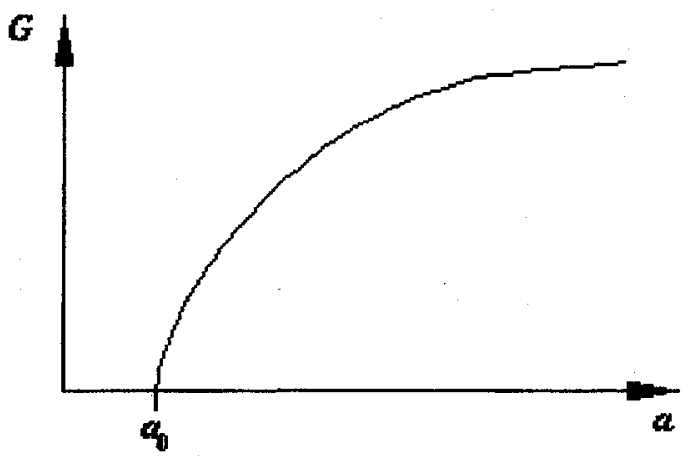

Figure 4.8. Crack growth resistance curve.

A common form of the curve is an exponential expression given by

$$
G=\lambda\left(a-a_{0}\right)^{n}+R_{1}
$$

where $\lambda, R_{1}$, and $n$ are material parameters and $a_{0}$ is the initial crack half width. The Mode I and Mode II stress intensity factors for the interface crack can be calculated from the interface stress state as follows

$$
\begin{array}{ll}
K_{I}=\sigma_{0} \sqrt{\pi a} ; & \sigma_{0}>0, \\
K_{I I}=\tau_{0} \sqrt{\pi a}, &
\end{array}
$$

where $\sigma_{0}$ and $\tau_{0}$ are the far field Mode I and Mode $\Pi$ stresses respectively and $a$ is the current crack half width. The local strain energy release rate can then be calculated as follows

$$
G=\frac{K_{l}{ }^{2}+K_{u}{ }^{2}}{E}=\frac{\left(\sigma_{0} \sqrt{\pi a}\right)^{2}+\left(\tau_{0} \sqrt{\pi a}\right)^{2}}{E},
$$


where $E$ is the Young's Modulus of the material. Equation (4.79) is inverted to find the change in crack length

$$
\Delta a=\left(a-a_{0}\right)=\left(\frac{G-R_{1}}{\lambda}\right)^{\frac{1}{n}} .
$$

Then, for each time step, the change in crack length is calculated based on the calculated strain energy release rate at each interface. If the new crack length is greater than the previous crack length, the interface crack grows. If this growth causes the crack width to exceed the interface width, the interface fails.

For crack growth using the resistance curve method, as the size of the initial interface cracks decreases, the interface stresses required to grow the crack increases. If the initial crack half width is very small, the stresses required to grow the crack exceed the strength of the material being modeled. Because the method described above only includes fracture and does not account for gross material failure, a gross stress failure criterion has been included. The sole criterion for this method is given by

$$
\sigma_{0}>\sigma_{f},
$$

where $\sigma_{f}$ is the failure stress of the material. Then, when the normal stress acting on the interface exceeds the material failure stress, the interface fails. 


\section{Results}

In the previous section, a method for modeling discrete dynamic fracture of structures in the presence of a distribution of cracks was presented. In this section, the method is tested on several different structures and the results of the tests are presented.

To determine if the structural and dynamic components of the numerical model are within expected accuracy, the vibration of three simple structures have been modeled. To investigate the effects of a distribution of small cracks on the dynamic characteristics of a structure, the vibration of these three simple structures has been compared for the case with no interface cracks and with interface cracks included. An investigation into the discrete fracture model has been done by modeling the fracture of a Compact Tension Specimen and a Single Edge Notched Beam Specimen. In addition, a future application of this modeling technique has been investigated.

\subsection{Structural Dynamics with Distributed Cracks}

The vibrations of three simple structures with analytical solutions are modeled to determine the accuracy of the numerical formulation and the implementation and to demonstrate the effects of a distribution of cracks throughout the structure. As stated previously, the interface cracks add a term to the element load vector, but the resulting stiffness matrix is that of a standard plane element formulated from the $\mathrm{Hu}$ - Washizu 
energy principle. Therefore, exclusion of the interface cracks results in a standard plane element model.

\subsubsection{Axial Vibration of a Beam}

The axial vibration of the long slender beam in Fig. 5.1 has been numerically modeled with plane stress elements. The fundamental frequency of vibration, $\omega$, of a solid beam of length $L$ is given by

$$
\omega=\frac{1}{4 L} \sqrt{\frac{E}{\rho}},
$$

where $E$ is Young's modulus and $\rho$ is the mass density [Volterra and Zachmanoglou, 1965]. The mean displacement, $\delta$, is the average displacement of the end of the vibrating beam and corresponds to the end displacement if the load is applied slowly. The mean displacement is given by

$$
\delta=\frac{\mathbf{f} L}{A E}
$$

where $A$ is the area of the beam and $\mathbf{f}$ is the applied load.

The beam, shown in Fig. 5.1, has a length of 20 in., a height of 1 in. and a width of 0.1 in. It is fixed at one end and a load ramp is applied at the other end as shown. The material properties are typical of linear elastic mild steel with $E=30 \mathrm{x}$ $10^{6} \mathrm{psi}$ and $\rho=7.3 \times 10^{-4} \mathrm{lbf} \mathrm{sec}^{2} / \mathrm{in}^{4}$. Then, for the given material properties and dimensions, the fundamental frequency of the beam is $2534 \mathrm{~Hz}$, and the mean displacement is $1.33 \times 10^{-2} \mathrm{in}$. 

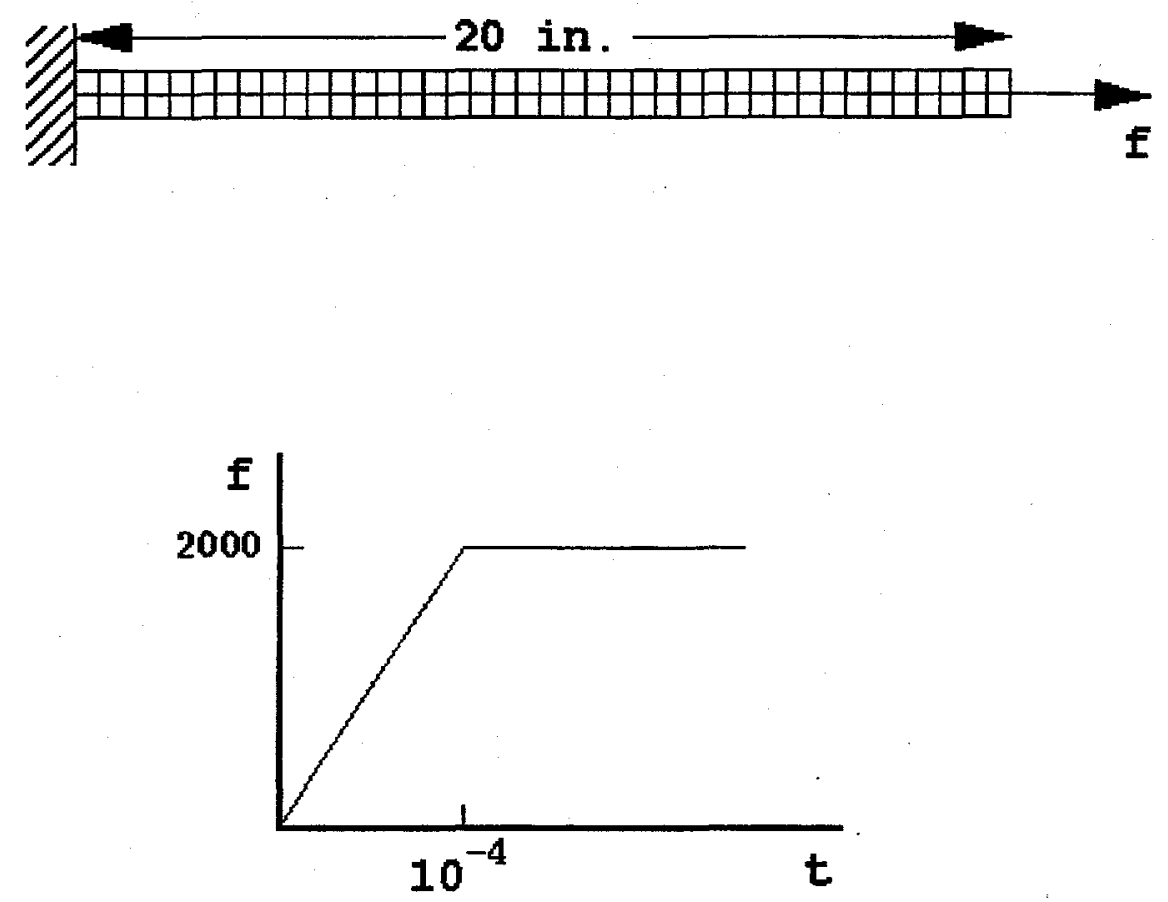

Figure 5.1. Model of a beam for axial vibration with a suddenly applied load.

The beam was discretized with 118 interface crack elements which consisted of 80 plane stress elements; 40 along its length and 2 through its height giving each element an aspect ratio of 1 . A comparison of axial displacement of the beam end for a model with no interface cracks and a model with interface cracks of length 0.4 in. is shown in Fig. 5.2. The vibration frequency of the beam with no interface cracks is determined to be $2532 \mathrm{~Hz}$, which is less than $0.1 \%$ from the theoretical value calculated above. This error is likely due to an error in determining the location of the peaks used to calculate the frequency and not an error in the numerical model. The frequency was determined by dividing the number of cycles of vibration by the time of vibration. The time of vibration is determined by locating the first and last peak of vibration. Because of the discrete nature of the analysis, the highest calculated point 
for each peak is not the true peak of vibration. This discrepancy between calculated and actual peak values is likely the major cause of error.

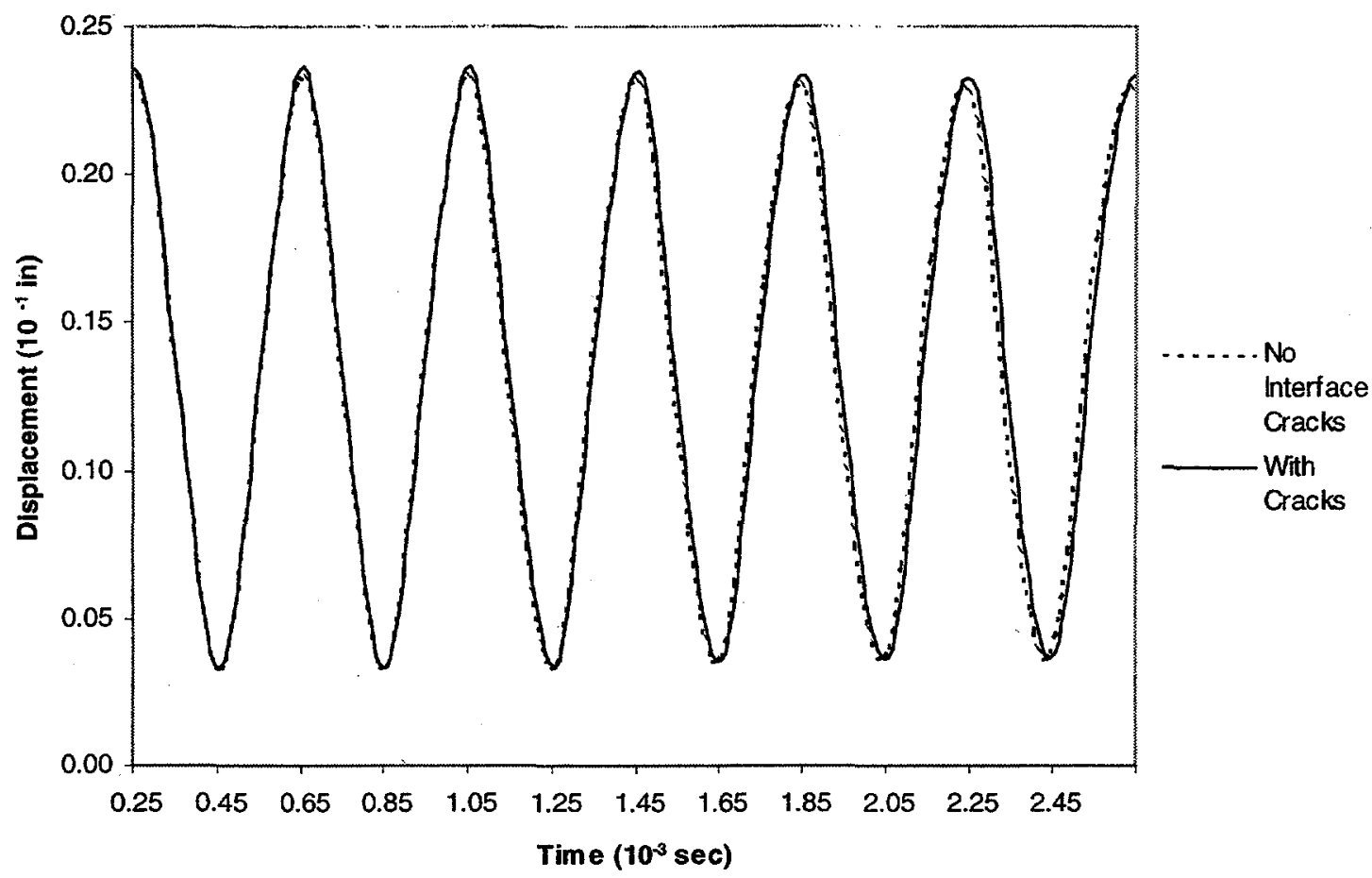

Figure 5.2. Comparison of axial displacement of a beam for the two models.

In comparison to the structural vibration model, the frequency of the model with interface cracks decreased to $2511 \mathrm{~Hz}$. Shown in Table 5.1, there is also a higher amplitude, $\alpha$, and mean deflection, $\delta$.

\begin{tabular}{ccccc}
\hline & $\begin{array}{c}\text { Theoretical } \\
\text { Value }\end{array}$ & $\begin{array}{c}\text { Model Without } \\
\text { Interface Cracks }\end{array}$ & $\begin{array}{c}\text { Model With } \\
\text { Interface Cracks }\end{array}$ & $\begin{array}{c}\text { Percent } \\
\text { Difference }\end{array}$ \\
\hline$\omega(\mathrm{Hz})$ & 2534 & 2532 & 2511 & $-0.84 \%$ \\
$\alpha$ (in.) & - & $9.801 \times 10^{-3}$ & $9.900 \times 10^{-3}$ & $1.01 \%$ \\
$\delta$ (in.) & $1.333 \times 10^{-2}$ & $1.336 \times 10^{-2}$ & $1.354 \times 10^{-2}$ & $1.39 \%$ \\
\hline
\end{tabular}

Table 5.1. Comparison of axial vibration of beam with and without interface cracks. 
For this type of problem, it is expected that the numerical results will be very close to the theoretical predictions. This is because the linear displacement along the length of the beam can be reproduced exactly by the linear displacement interpolation functions of the finite elements. The numerical results show good agreement between the theoretical predictions and the numerical model without interface cracks and are within the expected values. The inclusion of the interface cracks in the numerical model showed the expected trends. A distribution of small cracks in the beam would reduce the stiffness of the beam. This effect is shown in the numerical model by the decrease in frequency and increase in mean displacement and amplitude. It is expected that the effects of the interface cracks are small, but it is not known if the predicted magnitudes are correct.

\subsubsection{Transverse Vibration of a Cantilevered Beam}

The transverse vibration of a cantilevered beam has been modeled with plane stress elements. The first frequency of vibration of a long slender beam, neglecting shear and rotary inertia [Volterra and Zachmanoglou, 1965], is given by

$$
\omega=\frac{k^{2}}{2 \pi} \sqrt{\frac{E I}{\rho A}},
$$

where $I$ is the moment of inertia, $A$ is the area, and $k$ for fixed-free end conditions is given by

$$
k=\frac{\frac{\pi}{2}+0.30433078}{L} .
$$

$I$ and $A$ for a rectangular section are given by

$$
I=\frac{1}{12} b h^{3},
$$


and

$$
A=b h,
$$

where $b$ is the width and $h$ is the height. The mean displacement of the end of the beam is given by

$$
\delta=\frac{\mathbf{f} L^{3}}{3 E I}
$$

The beam, shown in Fig. 5.3, is 20 in. long, has a height of 1 in. and a thickness of 0.1 in. The material properties are the same as those given for the axial vibration. The first frequency of vibration from eqn. (5.3) is $81.9 \mathrm{~Hz}$, and the mean displacement is $5.33 \times 10^{-1}$ in.

The beam was discretized with 355 ICEs, which consisted of 200 plane stress elements; 40 lengthwise and 5 through its height giving each element an aspect ratio of 2.5. As with the axial vibration model, two models are compared. One model has no interface cracks and the second model includes interface cracks of length of 0.1 in.
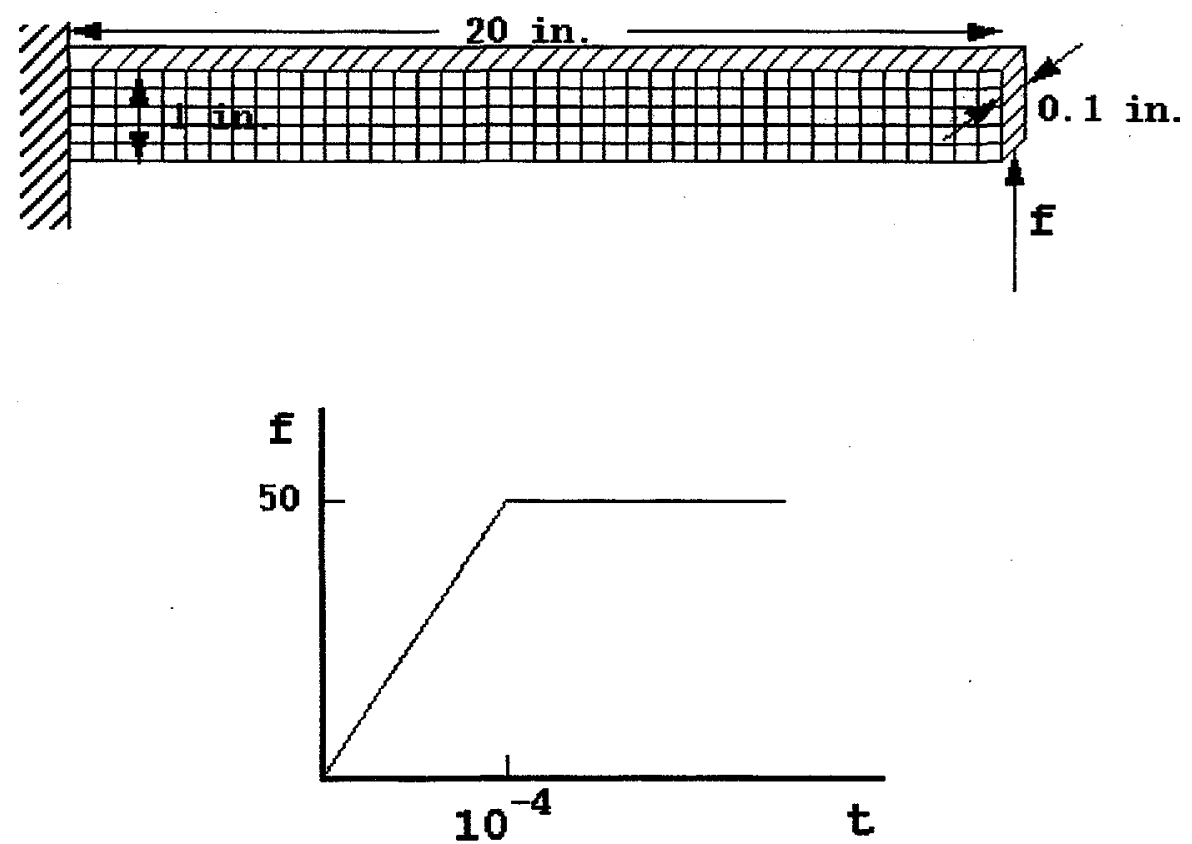

Figure 5.3. Model of a beam for transverse vibration with a suddenly applied load. 
The comparison of transverse displacement of the free end for both models is shown in Fig. 5.4. The frequency of vibration of the model with no interface cracks is determined to be $85.4 \mathrm{~Hz}$, which is a $4.27 \%$ difference from the value calculated from eqn. (5.3). This error is likely twofold; first, there is likely an error in the determination of the location of the vibration peaks, second, the numerical model uses linear displacement interpolation functions. Since strain is related to displacement by differentiation, the elements are only capable of modeling constant strain conditions. The strains are not constant in the cantilever beam problem, which has a linear distribution of strain through the height. Hence, given the model of five elements through the beam height, this linear distribution of strain is discretized into five different regions of constant strain. A greater number of elements through the height of the beam would yield better results.

In a comparison of the dynamic behavior of the two models, shown in Table 5.2 , the trends for the change in frequency and mean deflection are similar to the axial vibration. The decrease in amplitude seems to indicate a stiffening response, which is contrary to the softening indicated by the decrease in frequency and increase in mean displacement.

A comparison between the theoretical values and the numerically predicted results show that the model adequately predicts the dynamic characteristics of the cantilevered beam. Although there is a discrepancy between the two results, the numerical model is not expected to reproduce the physical solution as stated above. As with the axial beam vibration, the inclusion of interface cracks lowers the predicted frequency of vibration and increases the mean displacement as expected. The 
prediction of a decrease in amplitude is unexpected. This may be due to the discrete nature of the solution in which the predicted amplitude is not the true amplitude.

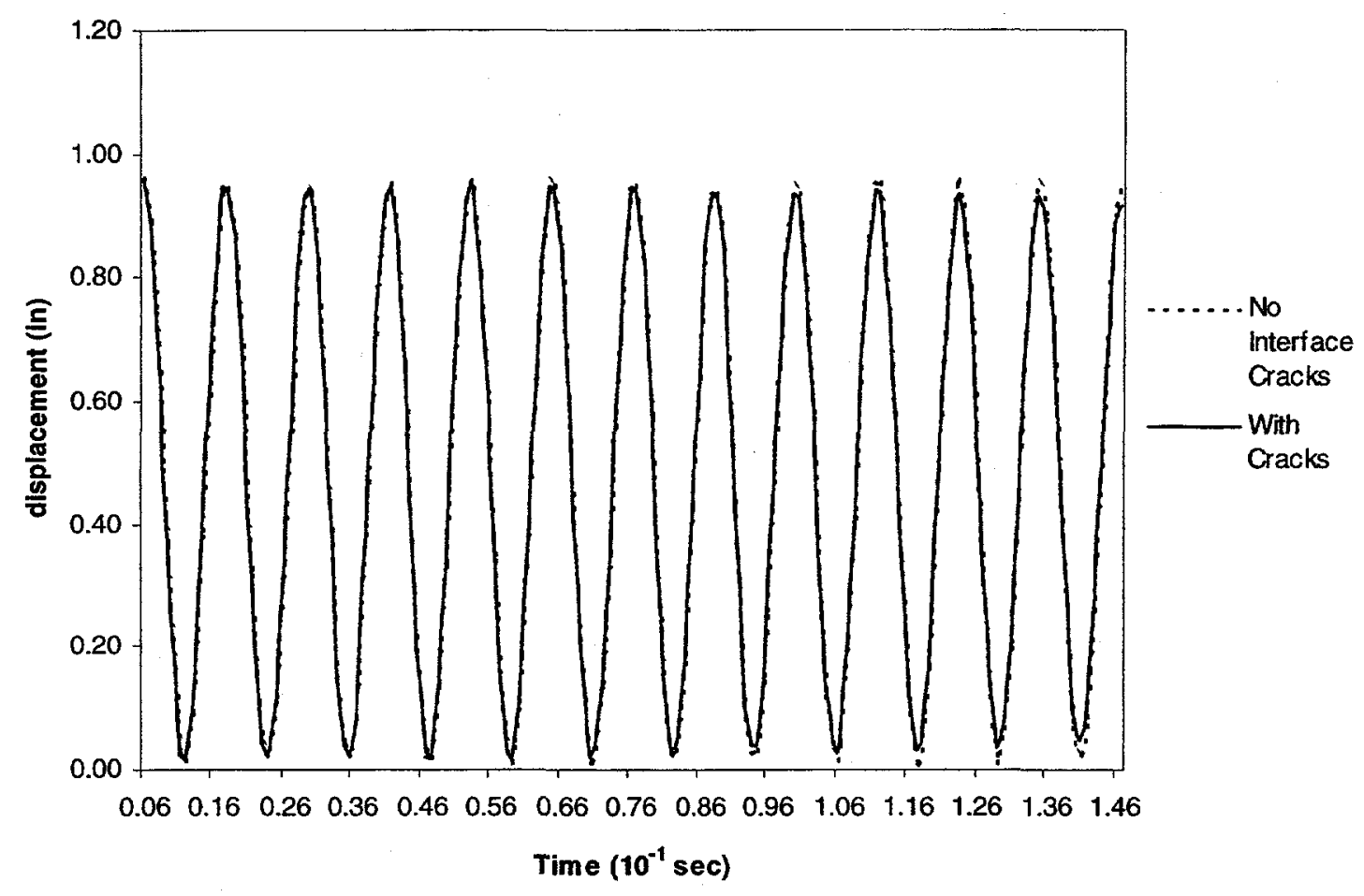

Figure 5.4. Comparison of transverse displacement of a beam for the two models.

\begin{tabular}{ccccc}
\hline & $\begin{array}{c}\text { Theoretical } \\
\text { Value }\end{array}$ & $\begin{array}{c}\text { Model Without } \\
\text { Interface Cracks }\end{array}$ & $\begin{array}{c}\text { Model With } \\
\text { Interface Cracks }\end{array}$ & $\begin{array}{c}\text { Percent } \\
\text { Difference }\end{array}$ \\
\hline$\omega(\mathrm{Hz})$ & 81.9 & 85.4 & 85.1 & $-0.35 \%$ \\
$\alpha($ in. $)$ & - & $4.65 \times 10^{-1}$ & $4.54 \times 10^{-1}$ & $-2.37 \%$ \\
$\delta$ (in.) & $5.33 \times 10^{-1}$ & $4.86 \times 10^{-1}$ & $4.87 \times 10^{-1}$ & $0.34 \%$ \\
\hline
\end{tabular}

Table 5.2. Comparison of transverse vibration of beam with and without interface cracks. 


\subsubsection{Vibration of a Thin Ring}

The first breathing mode of vibration of a thin ring has been modeled with plane stress elements. The breathing mode of vibration is the mode in which each circumferential segment expands and contracts radially at the same rate (i.e. the ring remains circular while expanding and contracting). The fundamental frequency of vibration is given by

$$
\omega=\frac{1}{2 \pi} \sqrt{\frac{E}{\rho r^{2}}},
$$

where $r$ is the mean radius [Timoshenko et al, 1974]. The mean displacement of the ring is given by

$$
\delta=\frac{\mathbf{P} r^{2}}{E t}
$$

where $t$ is the thickness of the cylinder and $\mathbf{P}$ is the internal pressure[Timoshenko and Woinowsky-Krieger, 1959].

The ring, shown in Fig. 5.5, has an inner radius of $4.0 \mathrm{in.}$ and an outer radius of 4.1 in. The material properties are the same as those given for the previous two examples. The first frequency of vibration from eqn. (5.8) is $7966 \mathrm{~Hz}$, and the mean displacement is $5.47 \times 10^{-3}$ in.

The ring was discretized with 540 ICEs, which consisted of 360 plane stress elements; 180 along its circumference and 2 through its radial dimension giving a minimum aspect ratio of about 2.8 and a maximum aspect ratio of about 2.9 . Two models are again compared; one without interface cracks and one with 0.002 in. cracks at each interface. 

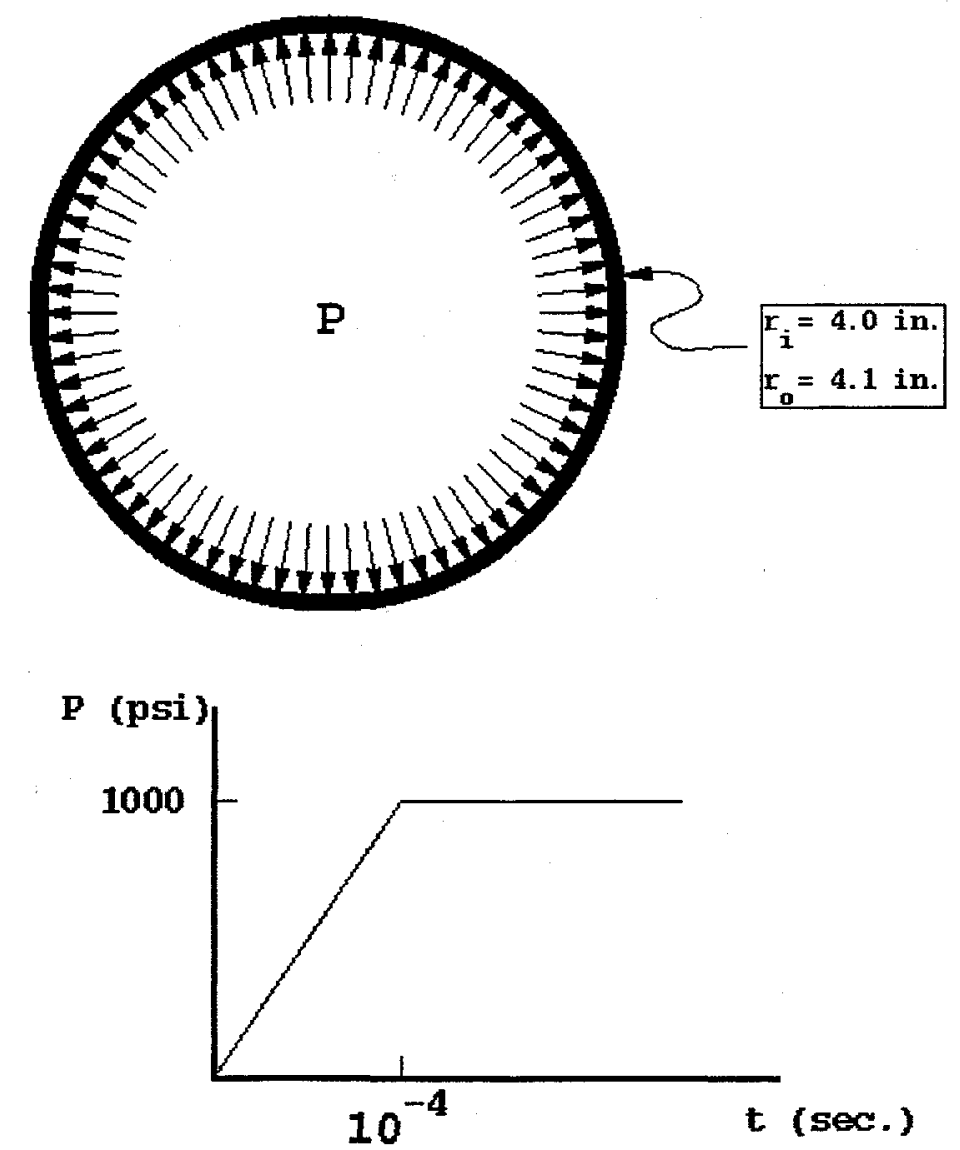

Figure 5.5. Model of a ring for vibration with a suddenly applied pressure.

A comparison of the radial displacement of the ring for the two models is shown in Fig. 5.6. The model predicts the vibration frequency without interface cracks to be $7937 \mathrm{~Hz}$, which is a $0.4 \%$ difference from the theoretical value, eqn. (5.8). As with the axial vibration model, the error is likely due to an error in determining the location of the peaks used to calculate the frequency and not an error in the numerical model. A comparison of the dynamic characteristics of the two models is shown in Table 5.3, where it can be noted that there are no obvious changes in the dynamic characteristics. This is encouraging because it is expected that the small size of the interface cracks in this model don't significantly affect the results. 


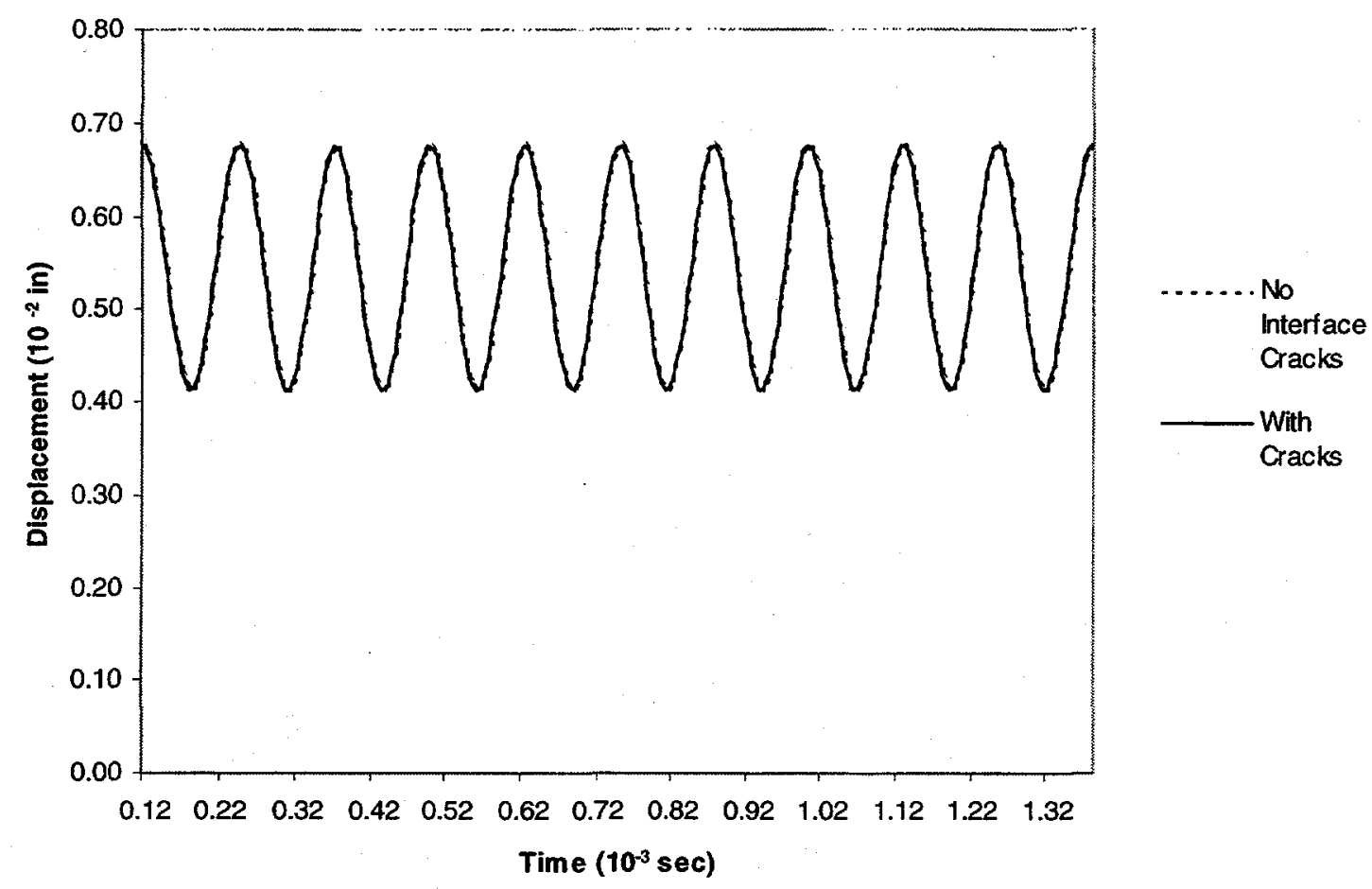

Figure 5.6. Radial displacement of a ring.

\begin{tabular}{ccccc}
\hline & $\begin{array}{c}\text { Theoretical } \\
\text { Value }\end{array}$ & $\begin{array}{c}\text { Model Without } \\
\text { Interface Cracks }\end{array}$ & $\begin{array}{c}\text { Model With } \\
\text { Interface Cracks }\end{array}$ & $\begin{array}{c}\text { Percent } \\
\text { Difference }\end{array}$ \\
\hline$\omega(\mathrm{Hz})$ & 7966 & 7937 & 7937 & $0.0 \%$ \\
$\alpha($ in. $)$ & - & $1.31 \times 10^{-3}$ & $1.31 \times 10^{-3}$ & $0.0 \%$ \\
$\delta$ (in.) & $5.47 \times 10^{-3}$ & $5.44 \times 10^{-3}$ & $5.44 \times 10^{-3}$ & $0.0 \%$ \\
\hline
\end{tabular}

Table 5.3. Comparison of vibration of a ring with and without interface cracks.

\subsection{Fracture Modeling}

A primary goal of this thesis is to provide a useful numerical engineering analysis tool that can, with relative ease, model the dynamic response of structures in the presence of discrete fracture with reasonable engineering accuracy. In Section 5.1, modeling of the structural response in the presence of interface cracks was 
demonstrated. In this section, several cases in which structural response and discrete fracture occur are presented. The results of the simulations demonstrate the effectiveness of the numerical algorithm and finite element implementation.

\subsubsection{Compact Tension Specimen}

The compact tension (CT) specimen is a well known and widely used specimen in fracture testing of metals [ASTM, 1997]. Properly configured, the testing of a CT specimen will yield the plane strain fracture toughness of the material. As used here, the relationship between the stress intensity factor and the applied load is inverted to determine the expected load at failure for a given geometry and material.

For the model presented here, the crack growth resistance curve is modeled as a sharp exponential given by

$$
G=213\left(a-a_{0}\right)^{\frac{1}{100}} .
$$

Figure 5.6 shows a graph of eqn. (5.10) where it can be seen that the strain energy release rate is approximately a constant of $200 \mathrm{in}-\mathrm{lb} / \mathrm{in}$. Therefore, the fracture toughness can be approximated by a constant with a value of $80 \times 10^{3} \mathrm{lbf} \sqrt{\text { in }}$. The material properties used in this investigation, given in Table 5.4, are typical of mild steel.

The nominal dimensions for the model are given in Fig. 5.7 with the actual dimension used in the model given in parentheses. The dimensions correspond to the required dimensions and allowable tolerances given in ASTM E399-90 for the material properties given in Table 5.4 [ASTM, 1997]. 


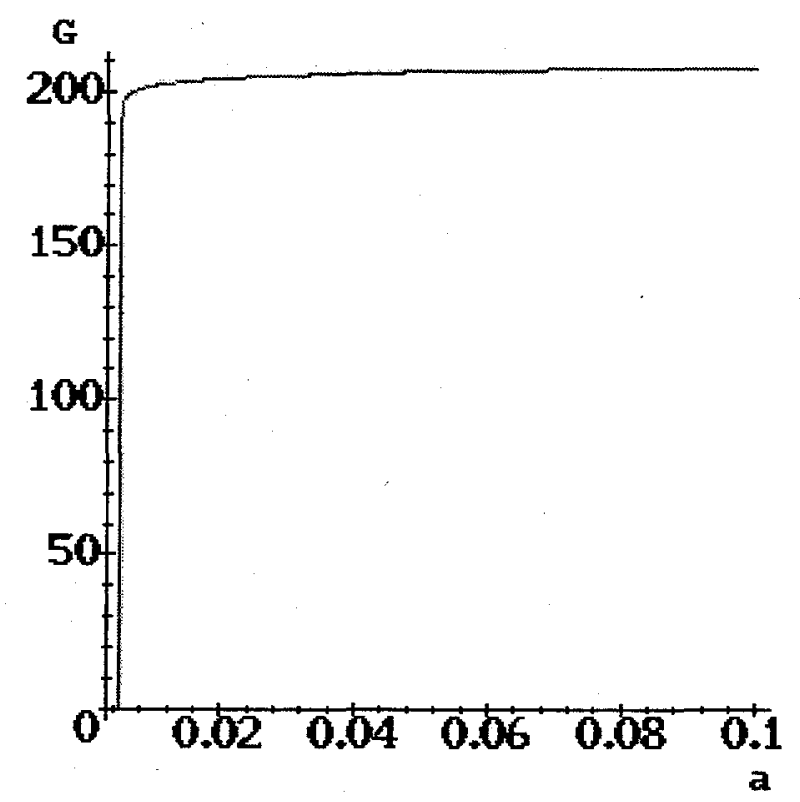

Figure 5.6. Crack growth resistance curve.

\begin{tabular}{|c|c|}
\hline Young's Modulus $(\mathrm{E})$ & $30 \times 10^{6} \mathrm{psi}$ \\
\hline Poisson's Ratio $(v)$ & 0.3 \\
\hline Yield Strength $\left(\sigma_{\mathrm{ys}}\right)$ & $80 \times 10^{3} \mathrm{psi}$ \\
\hline Fracture Toughness $\left(\mathrm{K}_{\mathrm{Ic}}\right)$ & $-80 \times 10^{3} \mathrm{psi} \sqrt{\mathrm{in}}$ \\
\hline$\lambda$ & $213 \mathrm{in}-\mathrm{lb} / \mathrm{in}$ \\
\hline$R_{1}$ & 0.0 \\
\hline $\mathrm{n}$ & 0.01 \\
\hline
\end{tabular}

Table 5.4. Material Properties for CT specimen.

The stress intensity factor, for the specimen in Fig. 5.7, is given by

$$
\mathrm{K}_{\mathrm{I}}=\frac{\mathrm{F}}{\mathrm{B} \sqrt{\mathrm{W}}} f(\mathrm{a} / \mathrm{W}) \text {. }
$$

Then, equating the stress intensity factor to the critical value, $\mathrm{K}_{\mathrm{I} c}$, and solving for the load at fracture,

$$
\mathrm{F}_{\mathrm{cr}}=\frac{\mathrm{K}_{\mathrm{Ic}} \mathrm{B} \sqrt{\mathrm{W}}}{f(\mathrm{a} / \mathrm{W})}
$$

where $f(0.518)=10.22$. Substitution into eqn. (5.12) gives a critical load of 43.5 kips. 


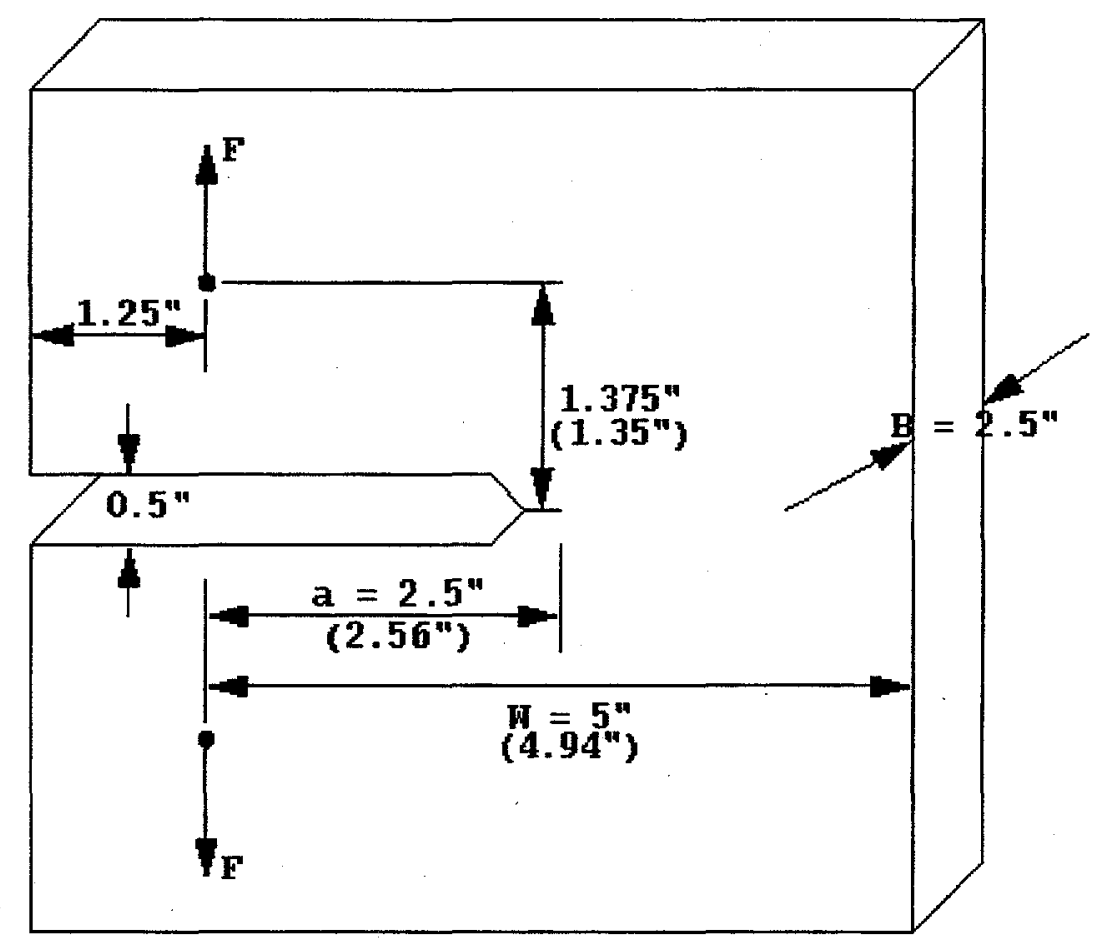

Figure 5.7. Nominal and actual dimensions for CT specimen model.

The finite element model, shown in Fig. 5.8, consists of 900 individual 2-D solid elements, 3600 nodes, and 1723 Interface Crack Elements. Each interface is seeded with a crack of length $0.01 \mathrm{in}$. The load is applied at a rate of $375 \mathrm{lbf} / \mathrm{sec} / \mathrm{in}$ ( $937.5 \mathrm{lb} / \mathrm{sec}$ ), which corresponds to 2.5 times the maximum load rate allowed by ASTM E399-90. For this analysis the time step starts at $5.0 \mathrm{sec} / \mathrm{step}$ and is reduced to a value of $1.28 \times 10^{-5} \mathrm{sec} / \mathrm{step}$ before the first fracture occurs. The time step is held constant at $1.28 \times 10^{-5} \mathrm{sec} / \mathrm{step}$ after the first ICE fracture.

Figure 5.9 shows a detail of the final crack growth pattern. All fractures in this model are due to the normal stress exceeding the failure stress of the material. The figure shows that the fracture propagates through 10 ICEs and then the crack branches, in a symmetrical pattern, away from the mid-plane. The numerical prediction of crack branching is likely an error due to the approximation of the stress state ahead of the 
crack tip. Although the prediction of crack branching might be an error, the crack path before branching (ICEs 1683-1692) is the expected path. Figure 5.10 shows four contour plots of the fracturing CT specimen. The contoured variable is the strain in the vertical direction (normal to the original sharp notch).

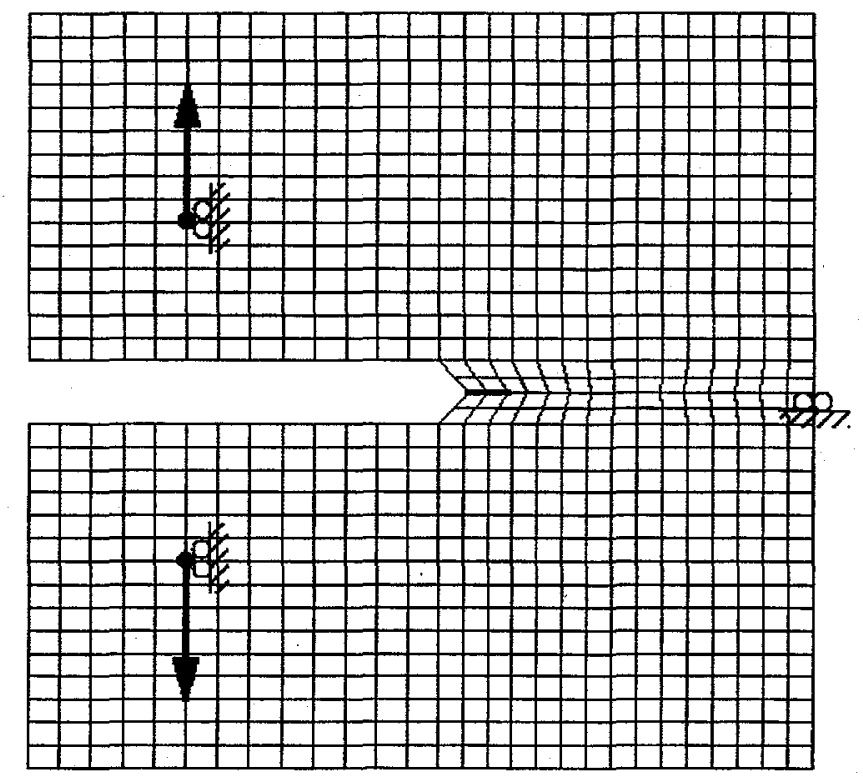

Figure 5.8. Mesh and boundary conditions for CT specimen model.

The failure time of each ICE is given in Table 5.5. From the failure times, the critical load value and crack propagation speed can be determined.

The first fracture occurs at $24.4000256 \mathrm{sec}$, which corresponds to a load of $22875 \mathrm{lbf}$. ICE number 1692 fractures at $24.4003584 \mathrm{sec}$, which corresponds to an increase in load of $0.312 \mathrm{lbf}$. This load is approximately $53 \%$ of the value of 43500 lbf given by eqn. (5.12). This error in approximating the failure loads is an area that needs further investigation. 


\begin{tabular}{cccc}
\hline ICE & Time $(\mathrm{sec}$ ) & ICE & Time $(\mathrm{sec}$.) \\
\hline 1683 & 24.4000256 & 822 & 24.4003584 \\
1684 & 24.4001280 & 836 & 24.4003584 \\
1685 & 24.4001408 & 1692 & 24.4003584 \\
1686 & 24.4001792 & 823 & 24.4003968 \\
1687 & 24.4002432 & 837 & 24.4003968 \\
1688 & 24.4002560 & 809 & 24.4004096 \\
1689 & 24.4002688 & 852 & 24.4004096 \\
1690 & 24.4002816 & 810 & 24.4004480 \\
821 & 24.4003200 & 853 & 24.4004480 \\
835 & 24.4003200 & 796 & 24.4005888 \\
1691 & 24.4003200 & 868 & 24.4005888 \\
\hline
\end{tabular}

Table 5.5. Failure times for ICEs in CT model.

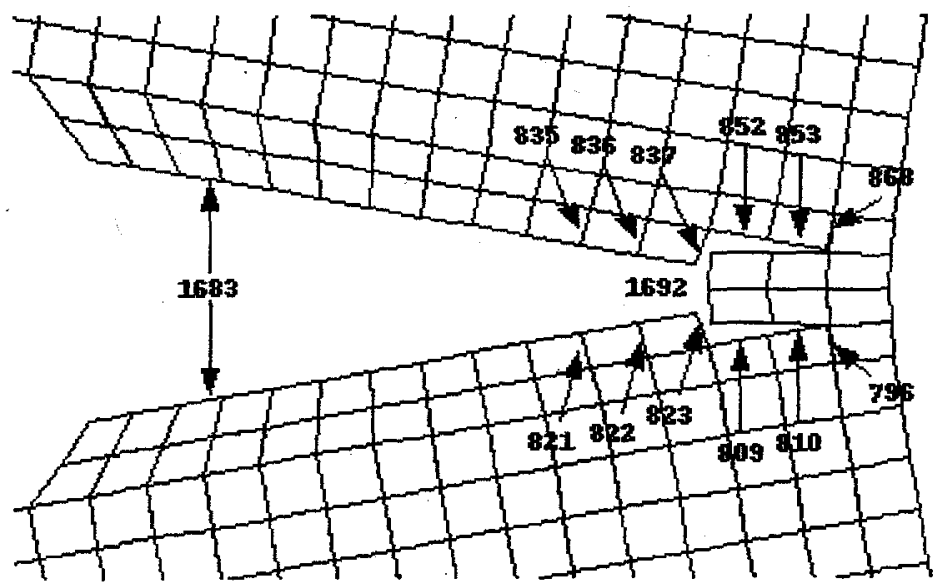

Figure 5.9. Detail of crack growth in CT specimen (10x magnification). 

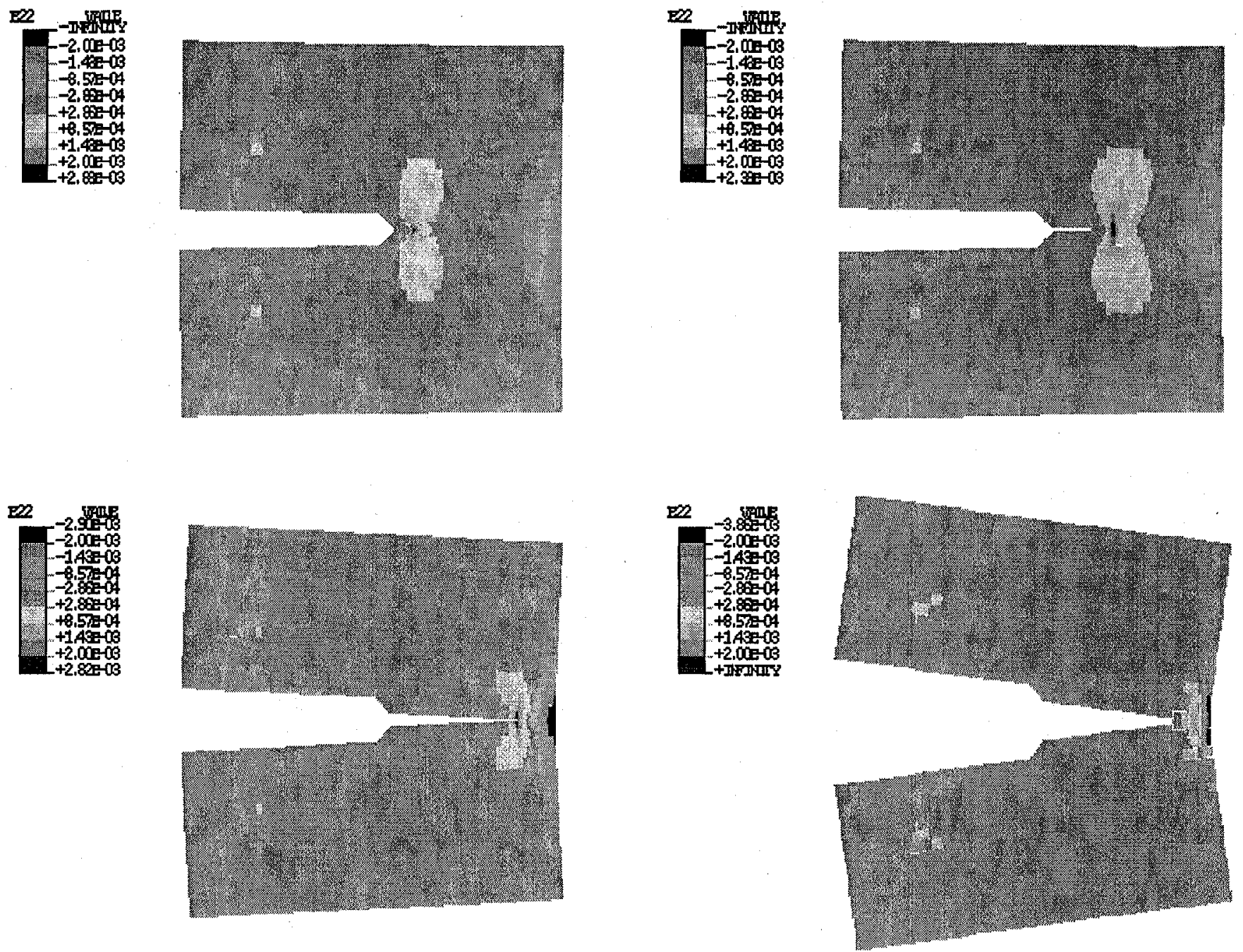

Figure 5.10. Contour of strain normal to sharp notch with 10x displacement magnification; Top left: before fracture, top right: 4 ICE failures, bottom left: 10 ICE failures, bottom right; crack branching. 
The speed of crack propagation is varied. Time between fracture of ICEs ranges from $1.024 \times 10^{-4} \mathrm{sec}$ to $1.28 \times 10^{-5} \mathrm{sec}$. This results in a crack velocity range of $1616 \mathrm{in} / \mathrm{sec}-15015 \mathrm{in} / \mathrm{sec}$. The average crack speed, not including the first ICE or the crack branching, is $8920 \mathrm{in} / \mathrm{sec}$. If the first two ICEs are not included, the average velocity is $9964 \mathrm{in} / \mathrm{sec}$. These values are well below the theoretical crack speed limit of the Rayleigh wave speed, $C_{R}$, and the experimentally observed values of maximum crack speed of approximately $0.4 C_{R}$. For the material properties used,

$$
C_{R} \approx 1.16 \times 10^{5} \frac{\mathrm{in}}{\mathrm{sec}}
$$

and

$$
0.4 C_{R}=4.64 \times 10^{4} \frac{\text { in }}{\mathrm{sec}}
$$

The maximum crack speed $(15015 \mathrm{in} / \mathrm{sec})$ in the numerical model is approximately $13 \%$ of theoretical limit and $32 \%$ of experimentally observed maximum crack speeds. The average crack speed ( $8920 \mathrm{in} / \mathrm{sec})$ is approximately $7.7 \%$ of the theoretical limit and $19 \%$ of experimentally observed maximum crack speeds.

The numerical model has predicted the expected crack paths, although the predicted failure load and crack speed are not reasonable. While it is encouraging that the correct failure path is predicted, the model needs work to accurately predict failure loads and speed.

\subsubsection{Single Edge Notched Beam}

Like the CT specimen, the single edge notched beam (SENB) is a specimen that is used to determine the plane strain fracture toughness of the test material. Standard testing requirements for the SENB specimen are given in ASTM E399-90. Similar to the fracture simulation of the CT specimen, the crack growth resistance 
curve used in the model is a sharp exponential so that the critical fracture toughness can be approximated by a constant. The $K_{\mathrm{I} c}-\mathrm{F}_{\mathrm{cr}}$ relationship can be inverted to determine the load at fracture for the test material. The properties for the SENB specimen material are given in Table 5.6.

\begin{tabular}{|c|c|}
\hline Young's Modulus $(\mathrm{E})$ & $30 \times 10^{6} \mathrm{psi}$ \\
\hline Poisson's Ratio $(v)$ & 0.3 \\
\hline Yield Strength $\left(\sigma_{\mathrm{ys}}\right)$ & $120 \times 10^{3} \mathrm{psi}$ \\
\hline Fracture Toughness $\left(\mathrm{K}_{\mathrm{I} c}\right)$ & $\sim 80 \times 10^{3} \mathrm{psi} \sqrt{\mathrm{in}}$ \\
\hline$\lambda$ & $213 \mathrm{in}-\mathrm{lb} / \mathrm{in}$ \\
\hline$R_{\mathrm{I}}$ & 0.0 \\
\hline $\mathrm{n}$ & 0.01 \\
\hline
\end{tabular}

Table 5.6. Material Properties for SENB specimen

Shown in Fig. 5.11 are the nominal and actual dimensions for the SENB specimen. These dimensions correspond to ASTM E399-90. The load is applied at a rate of $666.6 \mathrm{lbf} / \mathrm{sec}$, twice the maximum load rate specified in ASTM E399-90.

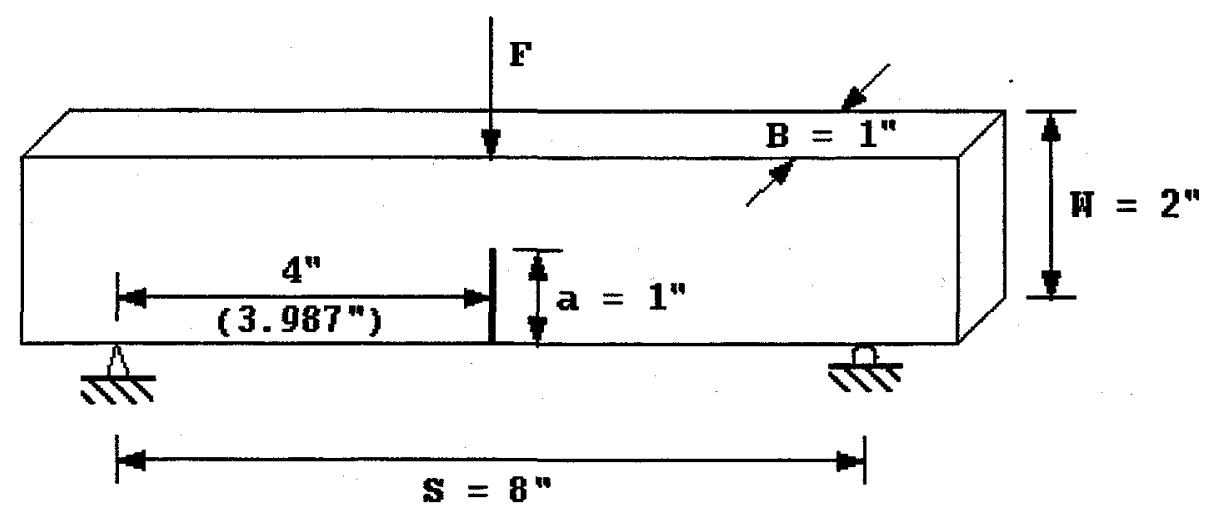

Figure 5.11. Nominal and actual dimensions for SENB specimen model. 
The stress intensity factor, for the specimen in Fig. 5.11, is given by

$$
\mathrm{K}_{\mathrm{I}}=\frac{\mathrm{FS}}{\mathrm{BW}^{\frac{3}{2}}} f(\mathrm{a} / \mathrm{W})
$$

Then, equating the stress intensity factor to the critical value, $\mathrm{K}_{\mathrm{I} c}$, and solving for the load at fracture,

$$
\mathrm{F}_{\mathrm{cr}}=\frac{\mathrm{K}_{\mathrm{Ic}} \mathrm{BW}^{\frac{3}{2}}}{\mathrm{~S} f(\mathrm{a} / \mathrm{W})},
$$

where $f(0.5)=2.66$. The load at fracture for the given material and geometry is 10.63 kips.

Two different meshes were used to model the SENB. The coarse mesh, shown in Fig. 5.12, consisted of 1836 interface crack elements constructed of 960 2-D solid elements with 3840 nodes. The mesh consisted of 16 equally sized divisions along the height (W in Fig. 5.11) and 60 element divisions along the length ( $\mathbf{S}$ in Fig. 5.11).

The length divisions are small near the center and transition to large divisions near the edges. The rows of elements on each side of the mid-plane are $0.070 \mathrm{in} . \times 0.125 \mathrm{in}$. and the rows of elements at the two ends are 0.263 in. $x 0.125$ in. Each interface is seeded with a crack having a width of 0.01 in.

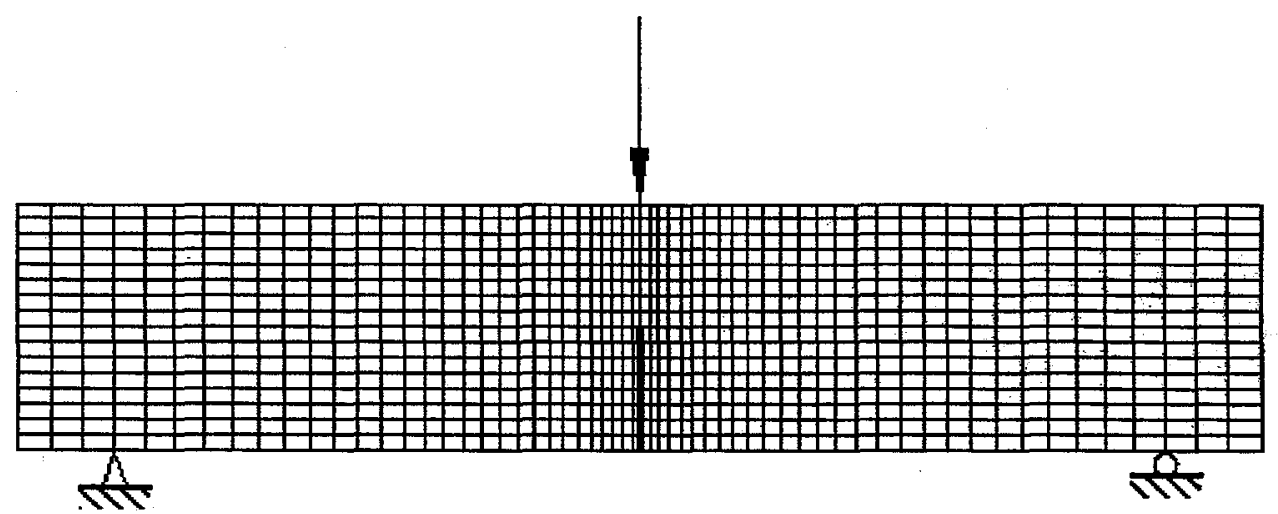

Figure 5.12. Coarse mesh of SENB specimen. 
The failure times of six elements in front of the crack is given in Table 5.7. The first ICE failure occurred at $15.417228 \mathrm{sec}$., which corresponds to a load of 10.277 kips. This value is $97 \%$ of the value given by eqn. (5.14). All ICE failures were a result of the normal stress at the interface exceeding the yield strength of the material and not a fracture of the interface crack. Beginning after the first ICE failure, the average crack speed for the fracture of the SENB specimen is $2713 \mathrm{in} / \mathrm{sec}$.

\begin{tabular}{cc}
\hline ICE & Time $(\mathrm{sec})$. \\
\hline 1829 & 15.4172288 \\
1830 & 15.4172672 \\
1831 & 15.4173056 \\
1832 & 15.4173952 \\
1833 & 15.4174336 \\
1834 & 15.4174592 \\
\hline
\end{tabular}

Table 5.7. Failure times for ICEs in the coarse mesh SENB model.

Figure 5.13 shows the displaced contour plot (displacement magnified 5 times) of four different states in the analysis. The contoured variable is the strain component along the horizontal direction (i.e. normal to the original sharp notch). Only six of the eight elements in front of the notch failed in the analysis. This is due to the difficulty in modeling the material state (displacement, strain and stress states) as the fracture nears the edge of the specimen. As mentioned previously, the strain field in a linear displacement element is constant, so that as the fracture nears the edge of the specimen, the decreasing number of elements through the thickness decreases the accuracy of the model. This results in an incorrect strain field in front of the crack and the crack no longer propagates in the expected direction. 

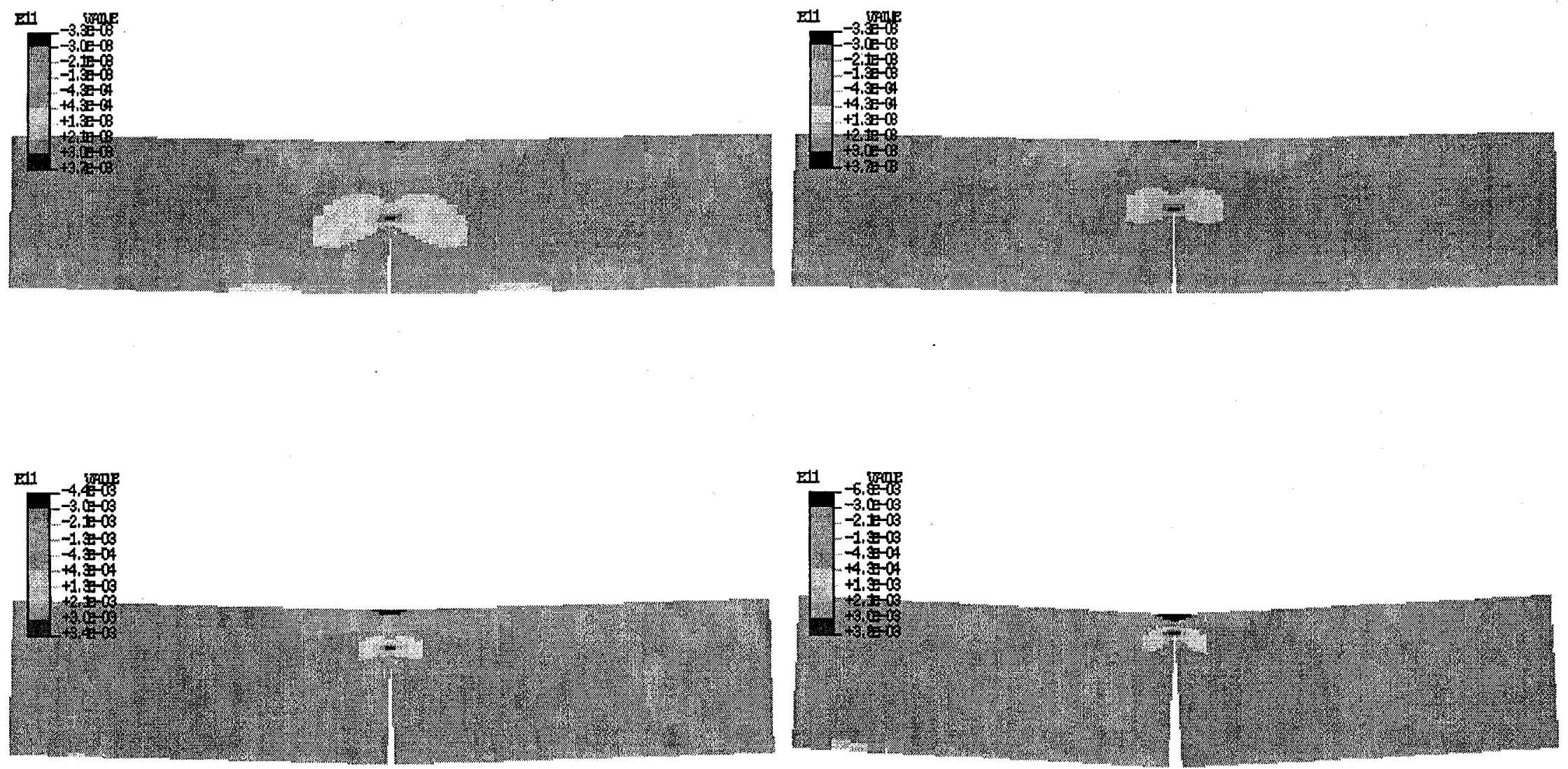

Figure 5.13. Contour of strain normal to sharp notch with $5 \mathrm{x}$ displacement magnification; Top left: before fracture, top right: 1 ICE failure, bottom left: 4 ICE failures, bottom right: 6 ICE failures. 
The fine mesh, shown in Fig. 5.14, consists of 3732 interface crack elements, 1920 2-D solid elements and 7680 nodes. The fine mesh has 32 equally sized divisions along the height and 60 element divisions along the length. The rows of elements on each side of the mid-plane are $0.070 \mathrm{in} . \mathrm{x} 0.0625 \mathrm{in}$. and the rows of elements at the two ends are 0.263 in. $x 0.0625$ in. As with the coarse mesh, the interface cracks have a width of $0.01 \mathrm{in}$.

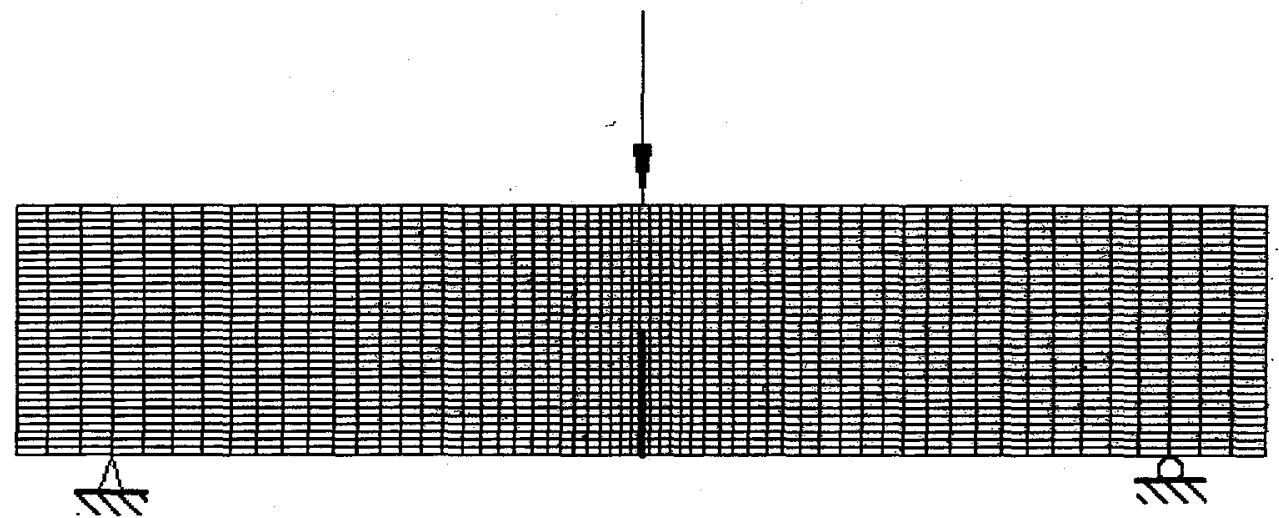

Figure 5.14. Fine mesh of SENB specimen.

The failure time of 14 elements in front of the crack is given in Table 5.7. The ICE directly in front of the sharp notch failed at $10.9712384 \mathrm{sec}$, which corresponds to a load of $7.313 \mathrm{kips}$. This value is $69 \%$ of the value given by eqn. (5.14). As with the coarse mesh model, all ICE failures were a result of the normal stress at the interface exceeding the ultimate strength of the material and not a fracture of the interface crack. The average speed of crack propagation for this model is $2048 \mathrm{in} / \mathrm{sec}$. 


\begin{tabular}{cccc}
\hline ICE & Time $(\mathrm{sec})$. & ICE & Time $(\mathrm{sec})$. \\
\hline 3717 & 10.9712384 & 3724 & 10.9714432 \\
3718 & 10.9712768 & 3725 & 10.9714560 \\
3719 & 10.9712896 & 3726 & 10.9714688 \\
3720 & 10.9713280 & 3727 & 10.9714944 \\
3721 & 10.9713664 & 3728 & 10.9715200 \\
3722 & 10.9714048 & 3729 & 10.9715712 \\
3723 & 10.9714176 & 3730 & 10.9716352 \\
\hline
\end{tabular}

Table 5.8. Failure times for ICEs in the fine mesh SENB model.

Figure 5.15 shows the displaced contour plot (displacement magnified 5 times) of four different states in the analysis. The contoured variable is the strain component along the horizontal direction (i.e. normal to the original sharp notch). With this model only 14 of the 16 elements in front of the notch failed in the analysis. As with the coarse mesh model, the remaining unfractured elements are due to the difficulty in correctly modeling the bending state with only two elements.

While both of the models for the SENB specimen predicted the expected crack propagation paths, only the coarse mesh accurately predicted the failure load. Both models predict crack speeds well below the expected values. The accuracy of both the predicted crack loads and crack propagation speeds decreased for the more refined mesh. This is counter intuitive to the expected increase in accuracy for a more refined mesh. 

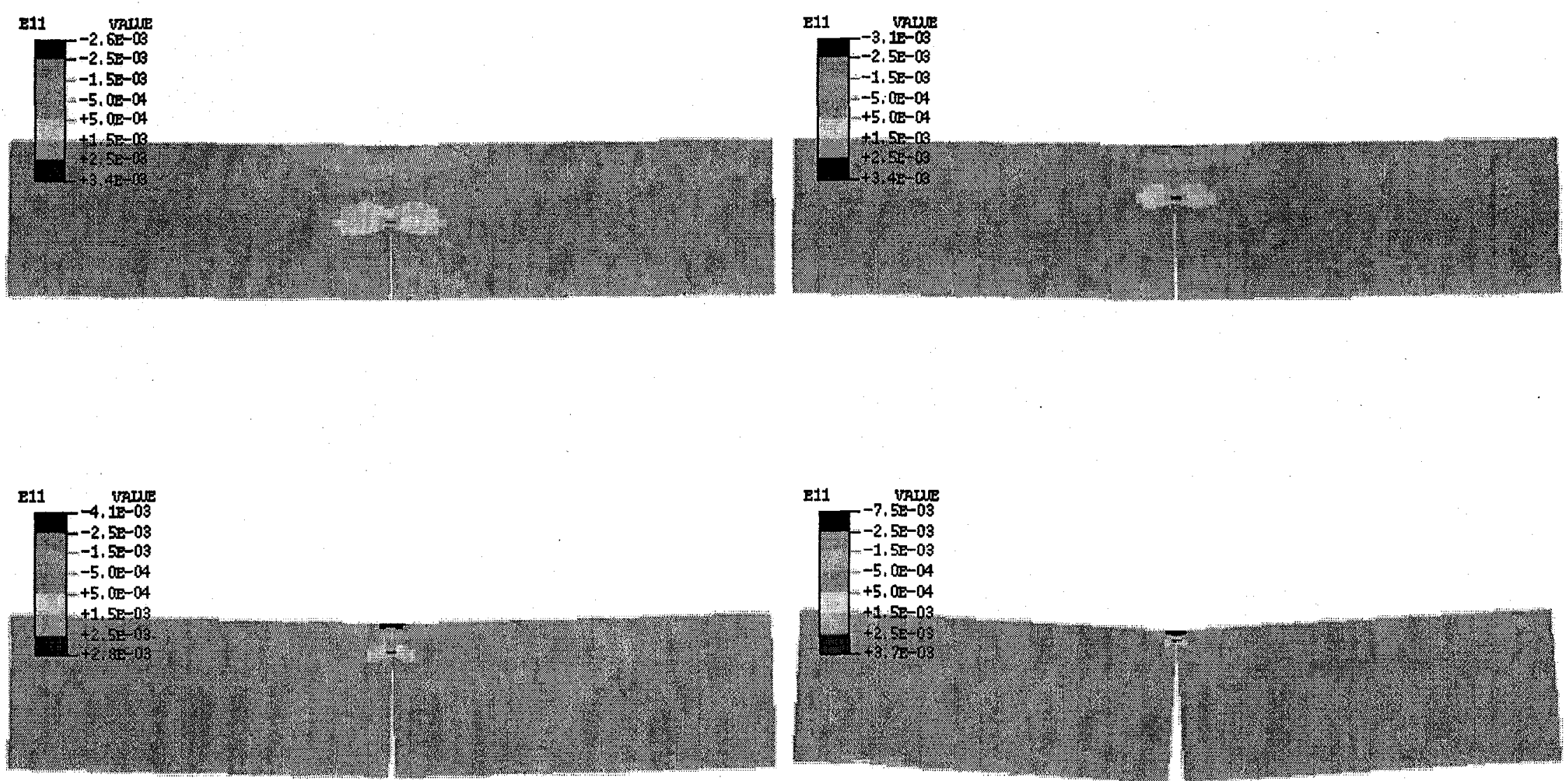

Figure 5.15. Contour of strain normal to sharp notch with $5 \mathrm{x}$ displacement magnification; Top left: before fracture, top right: 5 ICE failures, bottom left: 10 ICE failures, bottom right: 14 ICE failures 


\subsection{A Future Application}

Energetic particulate materials, or high explosives (HE), are used in many applications. Because the unexpected initiation of HE could potentially be a problem, the initiation behavior of $\mathrm{HE}$ under a variety of conditions is of great interest. The mechanically coupled cookoff (MCCO) of $\mathrm{HE}$ is an experiment designed to investigate the initiation behavior of HE subject to an explosive shock at elevated temperatures [Dickson et al, 1998].

In the MCCO experiments, a small flat cylinder of $\mathrm{HE}$ is confined in a 1/8 in. thick copper shell as shown in Fig. 5.16. The HE specimen has a 1 in. outer diameter with a 1/8 in inner diameter. The specimen is also confined on the top and bottom by glass, ceramic or sapphire as shown. The temperature of the HE is elevated to simulate cookoff and then the inner surface of the HE cylinder is shocked with a pressure pulse created by an explosive wire.

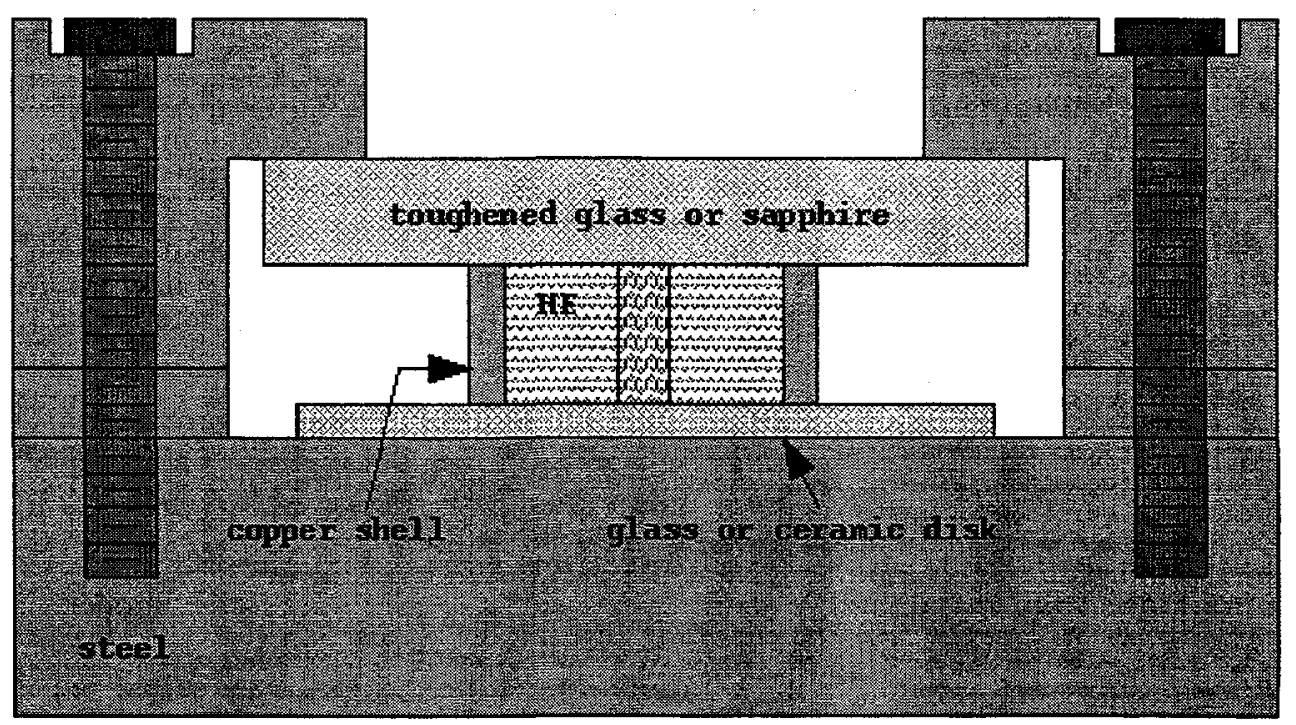

Figure 5.16. Cross-section of experimental setup of mechanically coupled cookoff of high explosives. 
Shown in Fig. 5.17, the resulting progression of events is viewed through the segment of toughened glass or sapphire used to confine the top of the HE cylinder. After the initiation of the pressure pulse on the interior of the HE cylinder, large radial cracks can be seen along with ignition on the newly exposed surfaces of the cracks.

The fracture patterns shown in Fig. 5.17 are typical of the results observed in the MCCO experiments, in which 3 large cracks occur with many small and micro cracks.
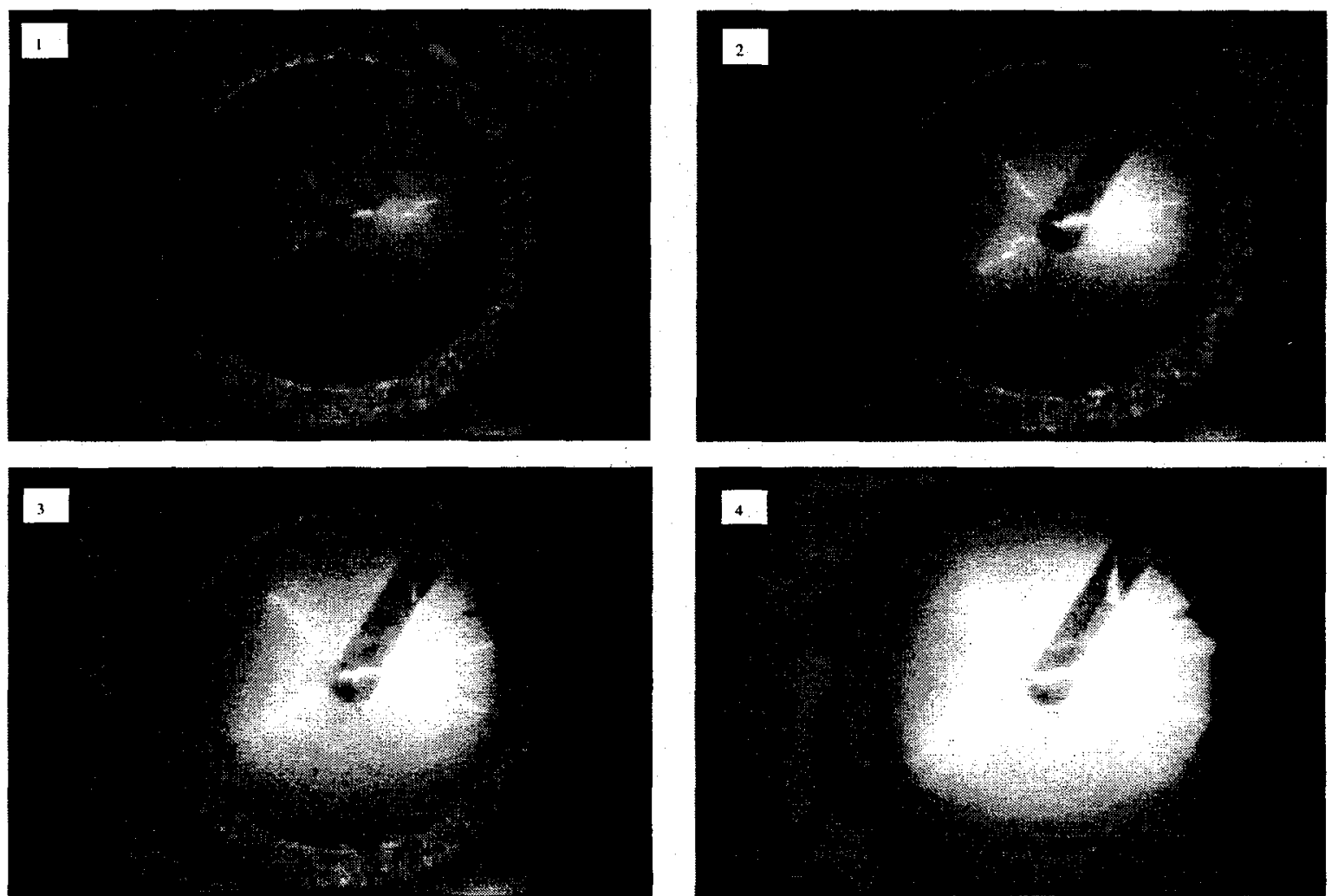

Figure 5.17. The progression of fracture and ignition in $\mathrm{MCCO}$ experiments.

The numerical modeling of these experiments is a difficult task. Many different phenomena must be incorporated into the model to accurately reproduce the results. The material, which is a particulate composite, is rate sensitive and undergoes 
damage due to the formation of microcracks. There are mismatches in thermal expansion coefficients, which results in the buildup of strain during an increase in temperature. The ignition in the HE material has significant temperature and material effects. The formation of macroscopic cracks and the exposure of new surface area requires that a discrete fracture model be used that can model these changes in geometry and correctly predict the formation of these cracks.

While all of these areas are being addressed, a complete model that can accurately predict the results of the MCCO experiments has not yet been achieved. One piece of this complete model is the discrete fracture method presented in this thesis. As an investigation into the application of the discrete fracture code to the MCCO experiments, a simple simulation of the MCCO has been undertaken.

The HE specimen and copper ring confinement were modeled as shown in Fig. 5.18. The outer copper ring is a simple plane strain linear elastic model with no cracks seeded at the element interfaces. The HE cylinder is a linear elastic plane strain model with a random distribution of cracks seeded at the element interfaces. The average crack length is 0.002 in. with a $50 \%$ random variation so that the cracks range in size from $0.001-0.003 \mathrm{in}$. The gross stress failure criterion is disabled so that failure of the ICEs is forced to be due to the interface crack growth exceeding the interface width. The interface between the $\mathrm{HE}$ and copper is a rigid bond.

Both materials are modeled as linear elastic materials with the properties for the HE being the author's best guess. The pertinent material properties are given in Table 5.9. The crack growth resistance curve is shown in Fig. 5.19. Because the curve is sharp and therefore, negligible crack growth occurs before sudden failure of 
the interface, a change in $\lambda$ would simply change the stress at which fracture occurs (e.g. a doubling of $\lambda$ would double the fracture stress).

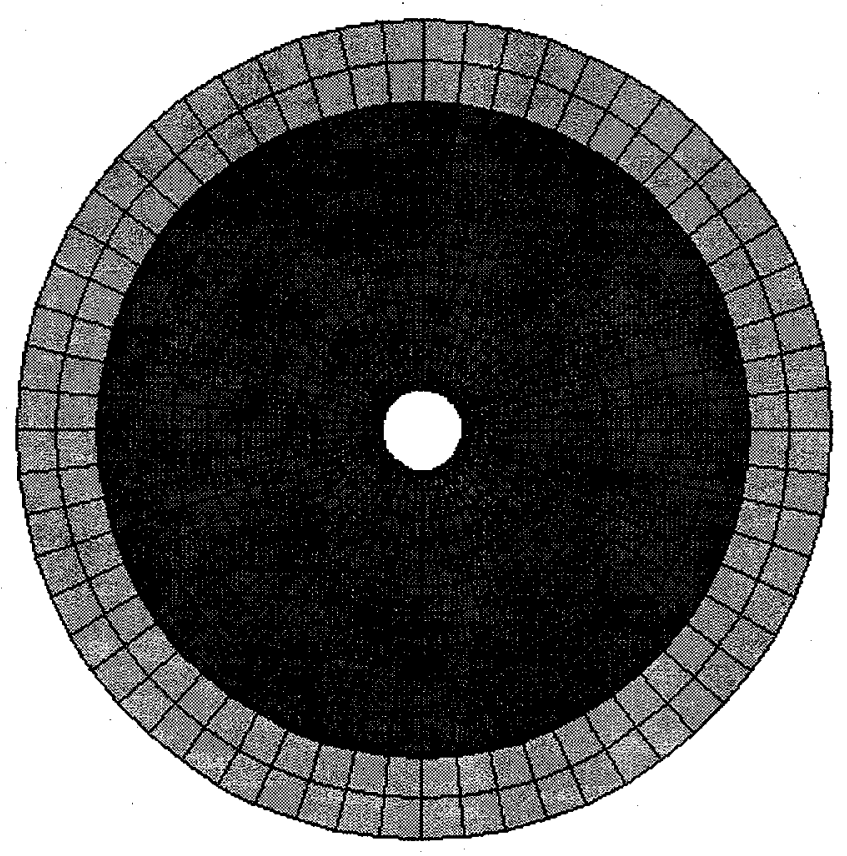

Figure 5.18. Model of HE and Copper in MCCO Experiments.

\begin{tabular}{|c|c|c|}
\hline & Copper & High Explosive \\
\hline Young's Modulus (E) & $2 \times 10^{7}$ & $5 \times 10^{6} \mathrm{psi}$ \\
\hline Poisson's Ratio $(v)$ & 0.33 & 0.3 \\
\hline$\lambda$ & - & $100 \mathrm{in}-\mathrm{lb} / \mathrm{in}$ \\
\hline$R_{1}$ & - & 0.0 \\
\hline $\mathrm{n}$ & - & 0.01 \\
\hline
\end{tabular}

Table 5.9. Material Properties for MCCO Model. 


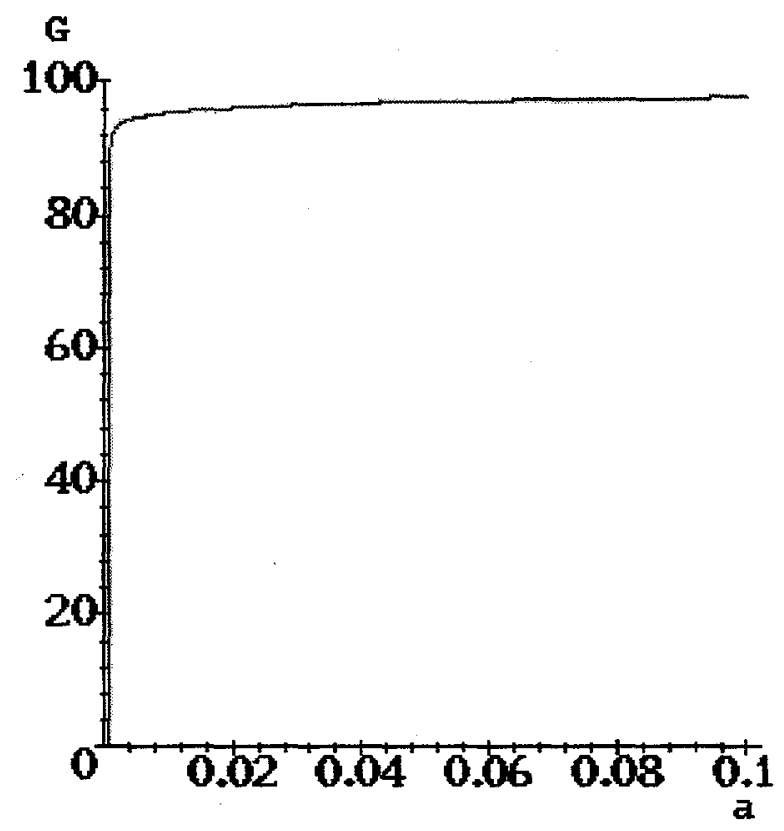

Figure 5.19. Crack growth resistance curve for $\mathrm{HE}$.

A load is applied to each node on the interior of the HE cylinder. Each load is initially perpendicular to the element faces to simulate an applied internal pressure. The direction of these forces remains constant so that if a fracture occurs, or the interior becomes non-circular for any other reason, the applied load is not a true pressure. In addition, this "pressure" is not applied to new crack surfaces when they form. The load is chosen so that fracture occurs at about $10^{-2} \mathrm{sec}$. This load has no relation to the load applied in the experiments.

Using these approximations and the mesh shown in Fig. 5.18, the analysis was performed. The results of the analysis, shown in the deformed wire frame mesh in Fig. 5.20, show that the general features of the MCCO experiments are reproduced. The two figures show two different analyses run under the same conditions. Each model has a different random distribution of interface cracks, but still in the range $0.001-0.003$ in. In each case, two large cracks form and propagate out towards the 
copper ring. Another, smaller crack, which doesn't propagate out to the copper ring within the analysis run times, also appears. Several small cracks also occur on the inner surface of the cylinder but don't propagate through more than 2-3 ICEs.

These results are very encouraging. Even with the gross approximations that have been made, the general features of the experimental results have been reproduced. The use of this discrete fracture model looks very promising for application in this area.
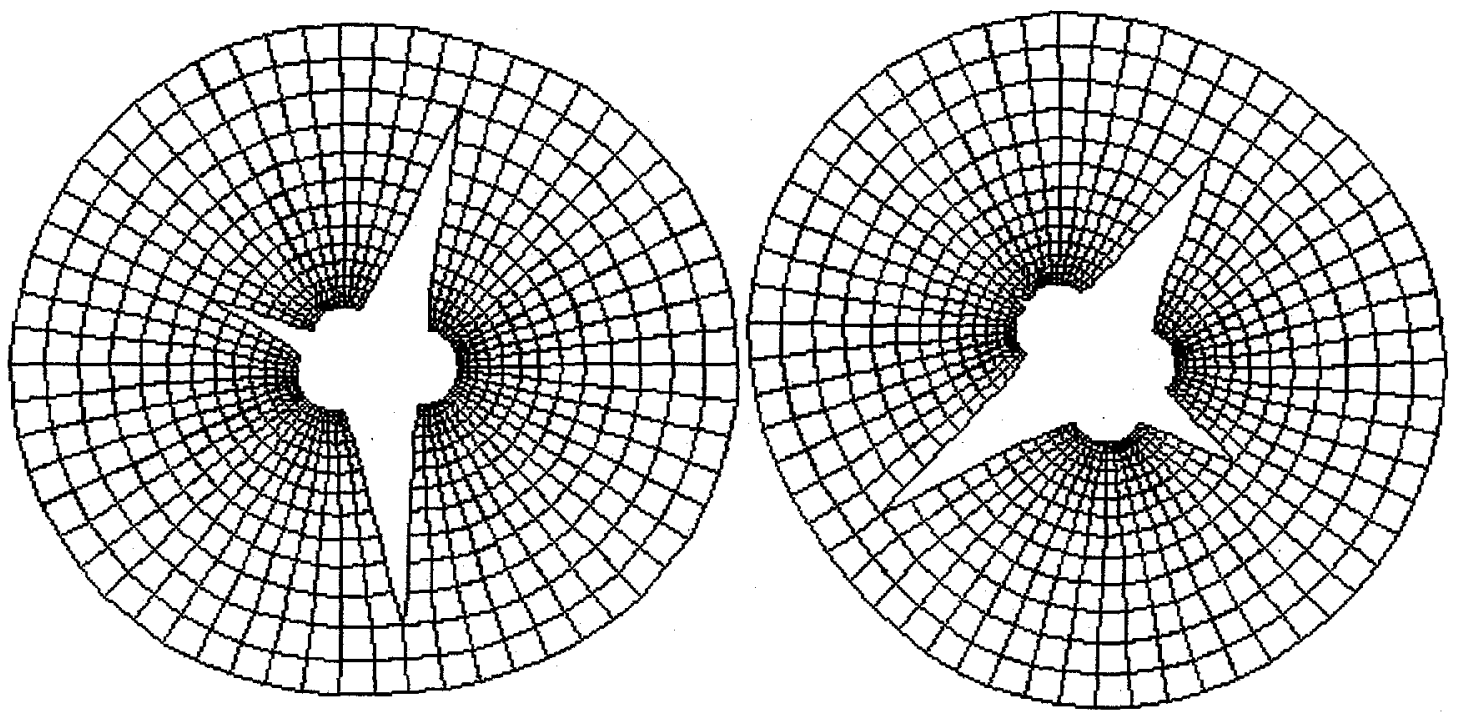

Figure 5.20. Two MCCO analyses. 


\section{Summary and Conclusions}

A method for modeling the discrete fracture of two-dimensional linear elastic structures with a distribution of small cracks subject to dynamic conditions has been developed. The foundation for this numerical model is a plane element formulated from the Hu-Washizu energy principle. The distribution of small cracks is incorporated into the numerical model by including a small crack at each element interface. The additional strain field in an element adjacent to this crack is treated as an externally applied strain field in the Hu-Washizu energy principle. The resulting stiffness matrix is that of a standard plane element. The resulting load vector is that of a standard plane element with an additional term that includes the externally applied strain field. Except for the crack strain field equations, all terms of the stiffness matrix and load vector are integrated symbolically in Maple V so that fully integrated plane stress and plane strain elements are constructed. The crack strain field equations are integrated numerically.

The modeling of dynamic behavior of simple structures was demonstrated within acceptable engineering accuracy. In the model of axial and transverse vibration of a beam and the breathing mode of vibration of a thin ring, the dynamic characteristics were shown to be within expected limits. The models dominated by tensile forces (the axially loaded beam and the pressurized ring) were within $0.5 \%$ of the theoretical values while the shear dominated model (the transversely loaded beam) 
is within $5 \%$ of the calculated theoretical value. The constant strain field of the tensile problems can be modeled exactly by the numerical model. The numerical results should therefore, be exact. The discrepancies can be accounted for by errors in the calculation of frequency from the numerical results. The linear strain field of the transverse model must be modeled by a series of constant strain elements. This is an approximation to the true strain field, so some error is expected.

The inclusion of interface cracks in the two beam models was shown to affect the dynamic response by decreasing the frequency of vibration and increasing mean displacement. Because the interface cracks in the thin ring model were small in comparison to the interface cracks in the beam models, the dynamic characteristics of the thin ring were not noticeably affected by the inclusion of interface cracks. For the axial and transverse beam models, the decrease in frequency was $0.84 \%$ and $0.35 \%$ respectively. The increase in mean displacement for the two models was $1.39 \%$ for the axial model and $0.34 \%$ for the transverse model. In addition to the decrease in frequency and increase in mean displacement, the amplitude of vibration increased for the axially loaded beam (an increase of $1.01 \%$ ) while it decreased for the transversely loaded beam (a decrease of $2.37 \%$ ). While these differences are small and could be well within the error range of these calculations, the general trends are qualitatively noticeable on the plot of axial displacement of the beam in Fig. 5.2. The trends are more pronounced in this model because the interface cracks are much larger than in the other two models.

These trends indicate a reduction in stiffness. A distribution of small cracks throughout a structure would have the affect of reducing the stiffness. Hence, the 
frequency changes and mean displacement changes that are shown to result from inclusion of interface cracks are expected. It has not been determined if the magnitudes of these changes are correct. An increase in the compliance would also increase the amplitude of vibration. The decrease in amplitude that is observed for the transverse beam vibration is unexpected and could be due to errors in locating the peaks of each cycle of vibration. The peaks are determined by finding the maximum displacement value in each cycle of vibration. The error results from the discrete nature of the solution process in which the maximum numerical value determined is not the true peak.

Discrete fracture has been accomplished by incorporating material failure criteria in the model and by defining each 2-D element so that it can separate from its neighbors without a redefinition of the element connectivity. Each element is defined by unique node numbers and then rigidly bonded to the neighboring elements to create the structure being modeled. If the failure criteria are met, this rigid bond is released and new crack surface is formed. This method allows for the creation of new crack surface at any of the element interfaces. Hence, new crack surface is not required to emanate from preexisting cracks and can initial and propagate along any element interface.

The material failure modes incorporated into the model are an elastic-plastic fracture model, based on a material's crack growth resistance curve, which includes a gross stress failure model. For the fracture failure model, the strain energy release rate at each element interface is calculated. Then a new interface crack length is calculated from the inverted crack growth resistance curve. If the interface crack grows wider 
than the element interface, failure occurs and the interface is allowed to separate. If the interface cracks are too small, the stress at failure using the crack growth resistance curve method exceeds the material failure stress. The gross stress failure criterion has been incorporated with the fracture failure model to correctly model material failure for very small interface cracks. The gross stress failure criterion defines failure as the normal stress exceeding the material failure stress.

Two standard fracture experiments were modeled to demonstrate the method. Compact Tension (CT) and Single Edge Notched Beam (SENB) specimens were modeled according to the geometrical specifications in ASTM E399-90. The CT specimen was loaded at the maximum rate specified and the two SENB specimens were loaded a rate twice the maximum specified.

The results showed that the numerical model can accurately predict the fracture paths, but does not predict the correct fracture load. The numerically predicted fracture path is correct until the crack tip stress field cannot be adequately modeled by the linear displacement finite elements. This occurs as the density of elements ahead of the crack tip decreases. For instance, as a crack nears the edge of a structure. For the CT specimen and the fine mesh SENB specimen, there was significant difference between the predicted critical load and the load at fracture in the numerical model. The CT specimen fractured at 53\% of the calculated value and the fine mesh SENB specimen fractured at $69 \%$ of the calculated value. The coarse mesh SENB model fractured at $97 \%$ of the calculated value. In all three cases, the failure was due to the normal stress exceeding the failure strength and not due to growth of the interface crack. The calculated crack velocities for the three cases were significantly below the 
expected crack velocities for rapid fracture. This is likely due to the differences between the numerical modeling of failure and the actual failure mode during fracture. For the numerical model, the failure must occur over an averaged distance in front of the crack tip, whereas the process zone for fracture in a real specimen is microscopic. It is not known how the crack velocities would be affected if the gross stress criterion were not used. This would allow for a failure by fracture of the interface crack, but the build-up of energy over an averaged finite distance in front of the crack tip would still be necessary.

A future application of this method has also been investigated. The mechanically coupled cookoff (MCCO) of high explosive (HE) is a well documented experiment. It was shown that, even with very gross approximation, the general crack features of the experiments have been reproduced.

More investigation of this method is still needed. The reason for the error in failure loads and crack propagation speeds needs to be investigated. The mesh sensitivity of the method needs to be determined. Studies need to be performed to determine if there is element size convergence and/or element size dependence. Possibly many other areas need to be investigated that have not yet been determined.

The method presented in this thesis has been shown to have promise for modeling the discrete dynamic fracture of structures with a distribution of small cracks. The mesh complexity is only slightly more complex than that of typical structural analysis. The failure criteria are based on a standard fracture mechanics model. Although the predicted failure loads and crack propagation speeds are in error, the crack propagation patterns appear to be correct. 


\section{REFERENCES}

E399 - 90, 1997 "Standard Test Method for Plane-Strain Fracture Toughness of Metallic Materials," American Society for Testing and Materials, Vol. 03.01, pp.408-438.

ABAQUS/Standard User's Manual, Vol. I - III, Version 5.7, 1997, Hibbit, Karlsson and Sorenson Inc.

ABAQUS Theory Manual, Version 5.7, 1997, Hibbit, Karlsson and Sorenson Inc.

Abraham, F. F., 1996, "Dynamics of Brittle Fracture with Variable Elasticity," Physical Review Letters, Vol. 77, pp. 272-275.

Abraham, F. F., 1997, "Portrait of a Crack: Rapid Fracture Mechanics Using Parallel Molecular Dynamics," IEEE Computational Science \& Engineering, Vol. 4, pp. 66-77.

Abraham, F. F., Brodbeck, D., Rafey, R., Rudge, W. E., 1994,"Instability Dynamics of Fracture: A Computer Simulation Investigation," Physical Review Letters, Vol. 73, pp. 272-275.

Abraham, F. F., Brodbeck, D., Rudge, W. E., Xu, X., 1997, “A Molecular Dynamics Investigation of Rapid Fracture Mechanics," Journal of Mechanics and Physics of Solids, Vol. 45, pp. 1595-1619

Abraham, F. F., Schneider, D., Land, B., Lifka, D., Skovira, J., Gerner, J., Rosenkrantz, M., 1997, "Instability Dynamics in Three - Dimensional Fracture: An Atomistic Simulation," Journal of Mechanics and Physics of Solids, Vol. 45, pp. 1461-1471.

Aliabadi, M. H., ed., 1995, Dynamic Fracture Mechanics, Computational Mechanics Publications, Boston, pp. 1-136.

Anderson, T. L., 1995, Fracture Mechanics: Fundamentals and Applications, $2^{\text {nd }}$ Edition, CRC Press, New York.

Banks-Sills, L., Sherman, D., 1989, “On Quarter-Point Three-Dimensional Finite Elements in Linear Elastic Fracture Mechanics," International Journal of Fracture, Vol. 41, pp. 177-196. 
Banks-Sills, L., Sherman, D., 1992, “On the Computation of Stress Intensity Factors for Three-Dimensional Geometries by Means of the Stiffness Derivative and JIntegral Methods," International Journal of Fracture, Vol. 53, pp. 1-20.

Belytschko, T., Lu, Y. Y., Gu, L., 1995, "Crack Propagation by Element-Free Galerkin Methods," Engineering Fracture Mechanics, Vol. 51, pp. 295-315.

Belytschko, T., Lu, Y. Y., Gu, Y., Tabbara, M., 1.995, "Element-Free Galerkin Methods for Static and Dynamic Fracture," International Journal of Solids and Structures, Vol. 32, pp. 2547-2570.

Belytschko, T., Organ, D., Krongauz, Y., 1995 "A Coupled Finite Element Element-Free Galerkin Method," Computational Mechanics, Vol. 17, pp. 186-195.

Belytschko, T., Tabbara, M., 1996, "Dynamic Fracture using Element-Free Galerkin Methods," International Journal for Numerical Methods in Engineering, Vol. 39, pp. 923-938.

Bennett, J. G., 1997, Personal Communication

Camacho, G. T., Ortiz, M., 1996, "Computational Modeling of Impact Damage in Brittle Materials," International Journal of Solids and Structures, Vol. 33, pp. 2899-2938.

Dickson, P.M., Asay, B.W., Henson, B.F., Fugard, C.S., 1998, "Observation of the Behaviour of Confined PBX 9501 Following a Simulated Cookoff Ignition," Proceedings, $11^{\text {th }}$ Detonation Symposium, Snowmass, CO.

De Borst, R., 1997, "Some Recent Developments in Computational Modelling of Concrete Fracture," International Journal of Fracture, Vol. 86, pp. 5-36.

Fleming, M., Chu, Y. A., Moran, B., Belytschko, T., 1997, "Enriched Element-Free Galerkin Methods for Crack Tip Fields," International Journal for Numerical Methods in Engineering, Vol. 40, pp. 1483-1504.

Freund, L. B., 1990, Dynamic Fracture Mechanics, Cambridge University Press, New York.

Goodman, R. E., Taylor, R. L., Brekke, T. L., 1968 " A Model for the Mechanics of Jointed Rock," Proceedings of the ASCE: Journal of the Soil Mechanics and Foundations Division, Vol. 98, pp. 637-659.

Hoff, R., Rubin, C.A., Hahn, G.T., 1986, “A New Finite-Element Technique for Modelling Stable Crack Growth”, Engineering Fracture Mechanics, Vol. 23, pp. 105-118. 
Hu, H.C., 1955, "On Some Variational Principles in the Theory of Elasticity and the Theory of Plasticity," Scientia Sinca, Vol. 4, pp. 33-54.

Kalia, R. K., Nakano, A., Omeltchenko, A., Tsuruta, K., Vashishta, P., 1997, "Role of Ultrafine Microstructures in Dynamic Fracture in Nanophase Silicon Nitride," Physical Review Letters, Vol. 78, pp. 2144-2147.

Lemaitre, J., 1986, "Local Approach of Fracture," Engineering Fracture Mechanics, Vol. 25, pp. 523-537.

Li, W., Kalia, R. K., Vashishta, P., 1996, “Amorphization and Fracture in Silicon Diselenide Nanowires: A Molecular Dynamics Study," Physical Review Letters, Vol. 77, pp. 2241-2244.

Li, Z., Reed, M., 1995, "A Finite Element Method to Model Progressive Fracture," Computer Methods in Applied Mechanics and Engineering, Vol. 120, pp. 303-313.

Li, F. Z., Shih, C. F., Needleman, A., 1985, "A Comparison of Methods for Calculating Energy Release Rates," Engineering Fracture Mechanics, Vol. 21, pp. 405-421.

Liaw, B. M., Kobayashi, A. S., Emery, A. F., 1984, "Double Noding Technique for Mixed Mode Crack Propagation Studies," International Journal For Numerical Methods in Engineering, Vol. 20, pp. 967-977.

Liebowitz, H., Sandhu, J. S., Lee, J. D., Menandro, F. C. M., 1995, “Computational Fracture Mechanics: Research and Application," Engineering Fracture Mechanics, Vol. 50, pp. 653-670.

Lotfi, H. R., Shing, P. B., 1995, "Embedded Representation of Fracture in Concrete with Mixed Finite Elements," International Journal for Numerical Methods in Engineering, Vol. 38, pp. 1307-1325.

Maple V Release 4, Version 4.00f, Waterloo Maple Inc.

Nakano, A., Kalia, R. K., Vashishta, P., 1995, "Dynamics and Morphology of Brittle Cracks: A Molecular-Dynamics Study of Silicon Nitride," Physical Review Letters, Vol. 75, pp. 3138-3141.

Needleman, A., 1987, “A Continuum Model for Void Nucleation by Inclusion Debonding," Journal of Applied Mechanics, Vol. 54, pp. 525-531.

Needleman, A., 1992, "Micromechanical Modelling of Interfacial Decohesion," Ultramicroscopy, Vol. 40, pp. 203-214. 
Needleman, A., 1997, "Numerical Modeling of Crack Growth Under Dynamic Loading Conditions," Computational Mechanics, Vol. 19, pp. 463-469.

Nishioka, T. 1997, "Computational Dynamic Fracture Mechanics," International Journal of Fracture, Vol. 86, pp. 127-159.

Nishioka, T. Stonesifer, R. B., Atluri, S. N., 1981, “An Evaluation of Several Moving Singularity Finite Element Models for Fast Fracture Analysis," Engineering Fracture Mechanics, Vol. 15, pp. 205-218.

Omeltchenko, A., Yu, J., Kalia, R. K., Vashishta, P., 1997, “Crack Front Propagation and Fracture in a Graphite Sheet: A Molecular-Dynamics Study on Parallel Computers;" Physical Review Letters, Vol. 78, pp. 2148-2151.

Parks, D. M., 1974, “A Stiffness Derivative Finite Element Technique for Determination of Crack Tip Stress Intensity Factors," International Journal of Fracture, Vol. 10, pp. 487-502.

Potapov, A. V., Campbell, C. S., 1996a, “A Hybrid Finite - Element Simulation of Solid Fracture," International Journal of Modern Physics C, Vol. 7, pp. 155-180.

Potapov, A. V., Campbell, C. S., 1996b, "A Three - Dimensional Simulation of Brittle Solid Fracture," International Journal of Modern Physics C, Vol. 7, pp. 717-729.

Potapov, A. V., Hopkins, M. A., Campbell, C. S., 1995a, “A Two - Dimensional Dynamic Simulation of Solid Fracture, Part I: Description of the Model," International Journal of Modern Physics C, Vol. 6, pp. 371-398.

Potapov, A. V., Hopkins, M. A., Campbell, C. S., 1995b, “A Two - Dimensional Dynamic Simulation of Solid Fracture, Part II: Examples," International Journal of Modern Physics C, Vol. 6, pp. 399-425.

Sandhu, R. S., Huang, S. W., 1975, "Application of Griffith's Theory to Analysis of Progressive Fracture," International Journal of Fracture, Vol. 11, pp. 107-121.

Siegmund, T., Fleck, N. A., Needleman, A., 1997, "Dynamic Crack Growth Across an Interface," International Journal of Fracture, Vol. 85, pp. 381-402.

Siegmund, T., Needleman, A., 1997, “A Numerical Study of Dynamic Crack Growth in Elastic-Viscoplastic Solids," International Journal of Solids and Structures, Vol. 7, pp. 769-787.

Sukumar, N., Moran, B., Black, T., Belytschko, T., 1997, “An Element-Free Galerkin Method for Three-Dimensional Fracture Mechanics," Computational Mechanics, Vol. 20, pp. 170-175. 
Timoshenko, S., Woinowsky-Krieger, S., 1959, Theory of Plates and Shells, McGrawHill, Inc., New York, p. 475.

Timoshenko, S., Young, D.H., Weaver, W. Jr. 1974, Vibration Problems in Engineering, 4th Edition, John Wiley and Sons, New York, pp. 476-481.

Vashishta, P., Nakano, A., Kalia, R. K., Ebbsjö, I., 1996, “Crack Propagation and Fracture in Ceramic Films - Million Atom Molecular Dynamics Simulations on Parallel Computers," Materials Science \& Engineering B-Solid State Materials for Advanced Technology, Vol. 37, pp. 56-71.

Volterra, E., Zachmanoglou, E. C., 1965, Dynamics of Vibrations, Charles E. Merrill Books, Inc., Columbus, pp. 293-303, 310-323.

Weissman, S. L., Taylor, R. L., 1992, "Treatment of Internal Constraints by Mixed Finite Element Methods: Unification of Concepts," International Journal for Numerical Methods in Engineering, Vol. 33, pp. 131-141.

Xu, X. P., Needleman, A., 1993, "Void Nucleation by Inclusion Debonding in a Crystal Matrix," Modelling and Simulation in Materials Science and Engineering, Vol. 1, pp. 111-132.

Xu, X. P., Needleman, A., 1994a, "Erratum; Void Nucleation by Inclusion Debonding in a Crystal Matrix," Modelling and Simulation in Materials Science and Engineering, Vol. 2, pp. 417-418.

Xu, X. P., Needleman, A., 1994b, "Numerical Simulations of Fast Crack Growth in Brittle Solids," Journal of the Mechanics and Physics of Solids, Vol. 42, pp. 1397-1434.

Xu, X. P., Needleman, A, Abraham, F. F., 1997, "Effect of Inhomogeneities on Dynamic Crack Growth in an Elastic Solid," Modelling and Simulation in Materials Science and Engineering, Vol. 5, pp. 489-516.

Zienkiewicz, O. C., Nakazawa, S., 1984, "On Variational Formulation and its Modifications for Numerical Solution," Computers \& Structures, Vol. 19, pp. 303-313. 


\section{APPENDIX}

1. ICE Implementation

1.1 Defining the Model

1.1.1 A Simple Structure

1.1.2 External Files

1.1.3 ABAQUS Input File

1.2 External Subroutines

1.2.1 Multi-Point Constraint

1.2.1.1 Two Node

1.2.1.2 Four Node

1.2.2 User Element

1.2.2.1 First Entry

1.2.2.2 Procedure Type

1.2.2.3 Subroutine ENTER

1.2.2.4 Interface Stress State and Failure

1.2.2.5 Structural Contributions

1.2.2.6 Interface Crack Contributions

2. Files

2.1 ABAQUS Input File

2.2 Maple Worksheet

2.3 Multi-Point Constraint Subroutine

2.4 User Element Subroutine 


\section{ICE Implementation}

An interface crack element has been developed and implemented in the implicit integration finite element code ABAQUS/Standard. This is accomplished by defining the behavior of an element in the user element subroutine (UEL) and constraining coincident nodes with the multi-point constraint (MPC) subroutine. These two subroutines completely define the model $^{7}$ so that ABAQUS is only used to assemble the global equations and solve for the nodal variables (displacement, velocity and acceleration). The following documentation includes a description of the use of the external subroutines used to define the element and provide the interface separation.

\subsection{Defining the model}

A model is defined to allow for the creation of new crack surface as failure criteria are satisfied. This model must allow for the separation along any of the element interfaces. To accomplish this, each 2-D solid element is defined by unique node numbers so that no redefinition of element connectivity is needed when one element separates from an adjacent element. Until failure of the interface, the coincident nodes are statically condensed so that the elements are rigidly held together.

\subsubsection{A Simple Structure}

A simple structure, discretized into 92 -D solid elements, is shown in Fig. 1.1. The structure is defined in the $x-y$ plane (i.e. a purely 2-D definition) and can be subjected to any admissible boundary conditions. The assembled structure bears a close resemblance to a standard mesh for a simple structural analysis. The main difference is that the current definition allows for the redefinition of the geometry as the analysis progresses. Until this occurs, the model behaves similar to a standard plane element model.

An exploded view of the assembly of the simple structure shown in Fig. 1.1 is depicted in Fig. 1.2. The plain numbers indicate nodes, the boxed numbers indicate the ABAQUS user elements (called Interface Crack Elements or ICEs), and the circled numbers indicate the individual 2-D solid elements. Each 2-D solid element is defined by unique node numbers so that the nine 2-D solid elements are defined by 36 nodes (an equivalent standard mesh would contain 16 nodes). For use in the UEL subroutine, each ICE is defined by the eight nodes of the two adjacent solid elements.

Then for the assembly of nine 2-D solid elements, there are 12 interface crack elements. It is not necessary for the ICE numbering scheme to follow a regular geometrical pattern as shown, but ICEs must be numbered consecutively starting at 1 .

\footnotetext{
${ }^{7}$ It is a simple matter to include ABAQUS elements and analysis procedures in the model. As discussed here, it is assumed the model is constructed only of user elements.
} 


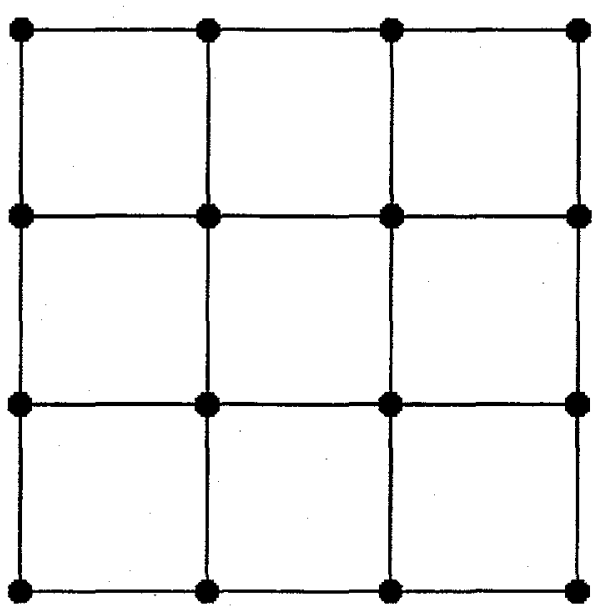

Figure 1.1. A simple assembly of elements.

For visualization purposes, each 2-D solid element is defined as a standard ABAQUS element with negligible thickness, stiffness and mass. For this definition, the standard elements must not have the same numbers as any of the ICEs.

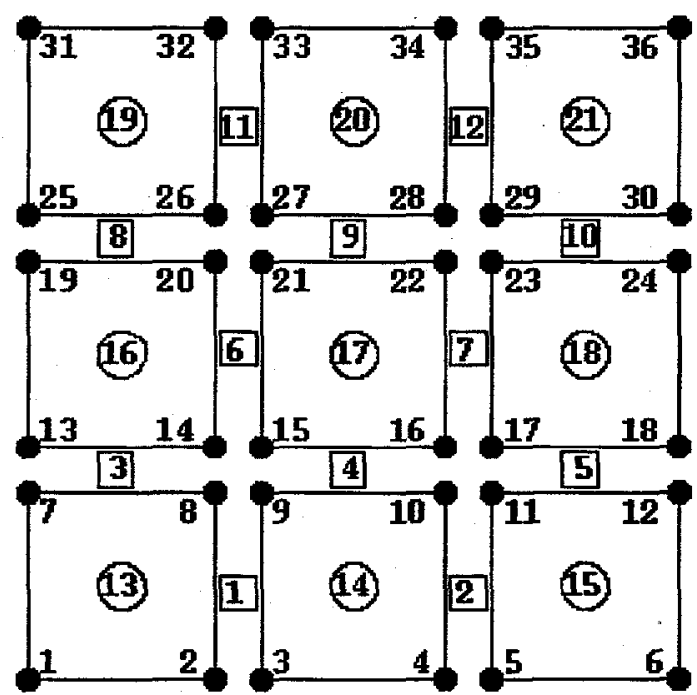

Figure 1.2. Exploded view of structure. 


\subsubsection{External Files}

Three different unformatted external files are read from the user element subroutine. These files are opened and read upon the first call into the subroutine. The values are stored in arrays so that the files need only be read once. These files provide the following information:

1. nodal thickness;

2. body forces;

3. adjacent solid elements for each ICE;

4. interface crack half width.

The thicknesses of the nodes defining each solid element are read from the file "thick" . For the assembly of Fig. 1.2, the file is given below. The number on the first line is the number of 2-D solid elements. The following lines give the solid element number ${ }^{8}$ along with the nodal thicknesses in the order the nodes are given in the ABAQUS input.

9

$\begin{array}{llllll}1 & 0.100 E+00 & 0.100 E+00 & 0.100 E+00 & 0.100 E+00 \\ 2 & 0.100 E+00 & 0.100 E+00 & 0.100 E+00 & 0.100 E+00 \\ 3 & 0.100 E+00 & 0.100 E+00 & 0.100 E+00 & 0.100 E+00 \\ 4 & 0.100 E+00 & 0.100 E+00 & 0.100 E+00 & 0.100 E+00 \\ 5 & 0.100 E+00 & 0.100 E+00 & 0.100 E+00 & 0.100 E+00 \\ 6 & 0.100 E+00 & 0.100 E+00 & 0.100 E+00 & 0.100 E+00 \\ 7 & 0.100 E+00 & 0.100 E+00 & 0.100 E+00 & 0.100 E+00 \\ 8 & 0.100 E+00 & 0.100 E+00 & 0.100 E+00 & 0.100 E+00 \\ 9 & 0.100 E+00 & 0.100 E+00 & 0.100 E+00 & 0.100 E+00\end{array}$

File 1.1. Thickness input file.

The $\mathrm{x}$ and $\mathrm{y}$ directional body forces for each solid element are read in from the file "body". The file is given below for the assembly of Fig 1.2 with no body forces.

9
$10.000 \mathrm{E}+00 \quad 0.000 \mathrm{E}+00$
$20.000 \mathrm{E}+00 \quad 0.000 \mathrm{E}+00$
$30.000 E+00 \quad 0.000 E+00$
$40.000 E+00 \quad 0.000 E+00$
$50.000 \mathrm{E}+00 \quad 0.000 \mathrm{E}+00$
$6 \quad 0.000 \mathrm{E}+00 \quad 0.000 \mathrm{E}+00$
$70.000 \mathrm{E}+00 \quad 0.000 \mathrm{E}+00$
$80.000 E+00 \quad 0.000 E+00$
$90.000 E+00 \quad 0.000 E+00$

File 1.2. Body force input file.

\footnotetext{
${ }^{8}$ The solid element numbers need not correspond to the numbers used for definition of the standard ABAQUS plane elements. The author has numbered the solid elements consecutively starting at 1 in the external input files. This is not necessary.
} 
The body force input file follows a format similar to that of the thickness input file. The first line is the number of solid elements, followed by the solid element number with the $\mathrm{x}$ and $\mathrm{y}$ body forces.

The adjacent solid elements and the half width of the crack at the interface are read from the file "vfedeck". Shown below, the first line of the file has the number of interface crack elements. The subsequent lines contain the ICE number followed by the two adjacent solid elements and the interface crack half width.

$12 \begin{array}{rlll}1 & 2 & 1 & 0.100 \mathrm{E}+00 \\ 2 & 3 & 2 & 0.100 \mathrm{E}+00 \\ 3 & 4 & 1 & 0.100 \mathrm{E}+00 \\ 4 & 5 & 2 & 0.100 \mathrm{E}+00 \\ 5 & 6 & 3 & 0.100 \mathrm{E}+00 \\ 6 & 5 & 4 & 0.100 \mathrm{E}+00 \\ 7 & 6 & 5 & 0.100 \mathrm{E}+00 \\ 8 & 7 & 4 & 0.100 \mathrm{E}+00 \\ 9 & 8 & 5 & 0.100 \mathrm{E}+00 \\ 10 & 9 & 6 & 0.100 \mathrm{E}+00 \\ 11 & 8 & 7 & 0.100 \mathrm{E}+00 \\ 12 & 9 & 8 & 0.100 \mathrm{E}+00\end{array}$

File 1.3. Adjacent elements and crack width input file.

\subsubsection{ABAQUS Input File}

The input file required by ABAQUS follows the standard format given in the ABAQUS User Manual. There are a few special requirements for the defining the model used here. The type of user element must first be defined, along with the number of nodes, the number of coordinates at each node, the number of real properties, the number of solution dependant state variables, and the number of integer properties. This definition is followed by the numbers of the active degrees of freedom. The following lines are used to accomplish this definition:

*USER ELEMENT, TYPE=U1， NODES=8， COORDINATES=2，PROPERTIES=5, VARIABLES $=23$, IPROPERTIES $=2$

1,2

The material properties for each ICE and an indication of plane stress or plane strain analysis are input as UEL properties. The input of these properties is listed in the order of Young's Modulus, Poisson's Ratio, Strain Energy Release Rate, mass density, ultimate strength, and analysis flag. The analysis flag is 1 for plane stress and 2 for plane strain. The lines of input are as follows:

*UEL PROPERTY, ELSET=ice01

$.30000 \mathrm{E}+08, .30000 \mathrm{E}+00, .21300 \mathrm{E}+03, .73000 \mathrm{E}-03, .10000 \mathrm{E}+06$, 
The method of defining user elements in ABAQUS is the element number is given followed by the defining node numbers. For use here, each element is defined by 8 nodes. The format required is as follows:

1. the nodes are listed in counterclockwise order around the two adjacent 2-D solid elements;

2. the first four nodes define one adjacent solid element;

3. the second four nodes define the other adjacent solid element.

To accomplish this there are two different ways the user element can be defined. For example, user element number 1, could be defined the following two ways:

\begin{tabular}{|c|c|c|c|c|c|c|c|}
\hline $\begin{array}{l}1, \\
\text { or }\end{array}$ & 3 & 4, & 10 & 9, & 8, & 7 & 1, \\
\hline 1 , & 8, & 7 & 1 & 2, & 3, & 4 & 10, \\
\hline
\end{tabular}

The multi-point constraints also require special consideration. The majority of the user defined MPCs simply rigidly connect two coincident nodes of adjacent solid elements. The degrees of freedom ( $x$ and $y$ displacement in this case) of the first node listed in the input are constrained to the degrees of freedom of the second node listed. In effect, the degrees of freedom of the first node are replaced by the degrees of freedom of the second node so that only one node remains in the analysis. Problems arise when many interfaces intersect. It is possible to remove all the degrees of freedom at a point. The user defined MPC are similar to the ABAQUS "tie" MPC. The concerns that arise from the use of this MPC are discussed in the ABAQUS User Manual.

To avoid these problems, special considerations have been made, the details of which will be discussed later. The input for the MPC is required so that these special considerations can be enforced. The format for input into ABAQUS requires that the MPC number (or type) be listed followed by the dependent node then the independent node or nodes. For the assembly in Fig. 1.2, there are two different configurations; the coincident nodes on the exterior, which have 2 nodes, and the coincident nodes on the interior, which have 4 nodes. The exterior nodes require no special consideration and be listed in the ABAQUS required format in any order. For example, the MPC for nodes 2 and 3 of ICE 1 could be either of the following:

*MPC, USER, MODE $=$ NODE

.

1. $3, \quad 2$

or $1, \quad 3$

$1, \quad 2, \quad 3$ 
where the first number corresponds to the ICE number and the second two numbers are the dependent and independent nodes respectively. Because both nodes 2 and 3 are not used in any other MPCs, they can be listed in any order. The MPCs for the internal nodes follow specific rules. Each interior "location" requires $4 \mathrm{MPCs}$ to correctly constrain the nodes. The order in which they are listed must follow a counter-clockwise order. The last MPC, of the 4 required, must include all 4 coincident nodes listed in counter-clockwise order. For example, at the intersection of ICEs $1,3,4$, and 6 , the nodes $8,9,14$, and 15 are coincident. The required format and list order for these MPCs is as follows:

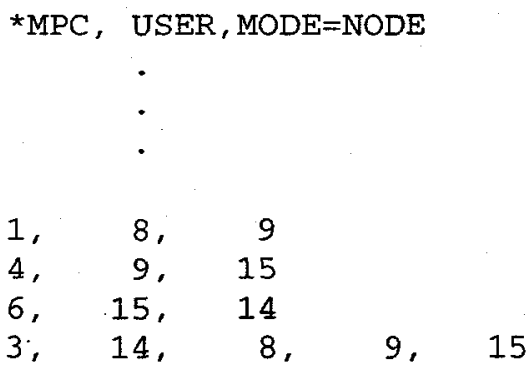

Note that the independent node of the each MPC becomes the dependent node of the following MPC. This is to satisfy the requirement that a dependant node cannot be constrained to another dependant node. These MPCs need not be together (i.e. on successive lines), but they must follow this pattern.

Special manipulations of the solution procedure are required to control the time step and to force the solution to iterate a minimum number of times. To change the time step during the analysis, the ABAQUS automatic time step algorithm must be enabled. A half-step residual tolerance (HAFTOL) must be given to do this. The purpose is to allow ABAQUS to choose a time step based on the magnitude of calculated half-step residual in comparison to the tolerance given. If the calculated value is larger than HAFTOL, the time step is reduced. For use here, the UEL subroutine completely controls the time step. Therefore, the value of HAFTOL must be set sufficiently large so that the time step is not reduced based on this criterion. The maximum value allowed by ABAQUS, and the value used by the author, is $1 \mathrm{x}$ $10^{36}$. The time step can be automatically increased if the solution is reached quickly. To negate this time step criterion, the convergence parameters for satisfying the field equation are modified. The values that are altered from their default settings are:

1. $R_{n}{ }^{\alpha}$, convergence criterion for the ratio of the largest residual to the corresponding average flux norm for convergence;

2. $C_{n}{ }^{\alpha}$, convergence criterion for the ratio of the largest solution correction to the largest corresponding incremental solution value;

3. $\tilde{q}_{u}{ }^{a}$, user defined average flux;

4. $R_{P}{ }^{\alpha}$, alternate residual convergence criterion used after $I_{P}{ }^{\alpha}$ iterations. 
$R_{n}{ }^{\alpha}$ is set to an unrealistically low number so that convergence is not reached based on this number. The value for $\tilde{q}_{u}{ }^{\alpha}$ is set to a value so that the ratio, $R_{P}{ }^{\alpha} / \tilde{q}_{u}{ }^{\alpha}$, is a suitable convergence criterion. $R_{P}{ }^{\alpha}$ is set to a value so that after $I_{P}{ }^{\alpha}$ iteration this criterion provides the "true" convergence criterion. $C_{n}{ }^{\alpha}$ is set to a suitable value that ensures a good solution based on this criterion. This value must be lower that the similar convergence criterion used in the UEL subroutine.

The time incrementation convergence parameters must also be adjusted to accommodate the model. The following time incrementation parameters are modified from their default values:

1. Io, equilibrium iteration after which a check is made whether the residuals are increasing in both of two consecutive iterations;

2. $I_{R}$, equilibrium iteration number at which logarithmic rate of convergence check begins;

3. $I_{p}$, equilibrium iteration after which alternate residual tolerance, $R_{p}$, is used;

4. $I_{C}$, maximum number of equilibrium iterations allowed;

5. $I_{L}$, number of equilibrium iterations above which the size of the next increment will be reduced;

6. $I_{G}$, maximum number of equilibrium iterations allowed in consecutive increments for the time step to be increased;

Because there can be significant changes in the problem during the coarse of an iteration, a large number of iterations may be required for convergence. Therefore, the values for $I_{o}, I_{R}$, and $I_{C}$, must be set sufficiently large to allow for convergence. Also, the automatic time incrementation parameter, $I_{L}$, is set to a high number to be effectively invalidated. The value of $I_{P}$ is then the minimum number of iterations before a check of convergence.

The following are the corresponding lines of input used to control the time incrementation in the user element subroutine:

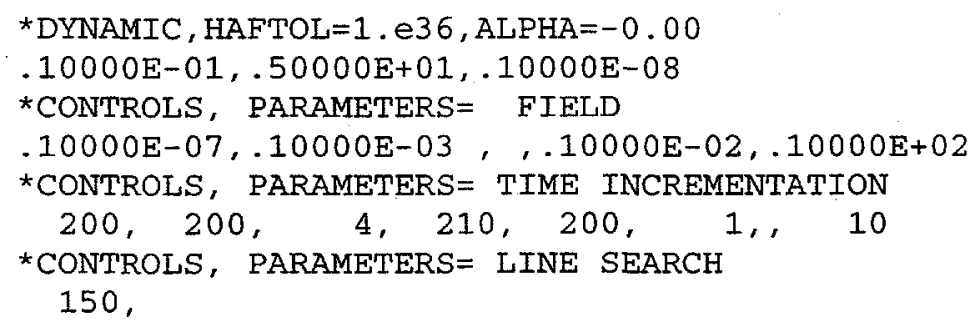

To have the node numbers that correspond to each ICE available in the UEL subroutine, the author has input these as field variables. The input for field variables has the format of node number followed by the values of all the field variables at that node. As used here, the only field variable at each node is the node number itself. 
Hence, the input is the following:

$\begin{array}{cc}\text { *FIELD } \\ \cdot \\ \cdot \\ \cdot \\ 5, \quad 5, \\ 6, & 6, \\ 7, & 7, \\ 8, & 8, \\ 9, & 9, \\ 10, & 10,\end{array}$

As a useful note, the interface crack half-width, and interface stresses sigO, sig1, and tau 0 are stored as solution dependent state variables. If post-processing capabilities are desired, it may be beneficial to include these in the values written to the ABAQUS restart file. A complete ABAQUS input file for the structure shown in Fig 1.2 is included in the APPENDIX. The input file is for a $1 \times 1 \mathrm{in}$. thin steel plate subject to fixed boundary condition along the lower edge and a load ramp along the upper edge.

\subsection{External Subroutines}

\subsubsection{Multi-Point Constraint}

There are two different multi-point constraints (MPC) used in this work; a two node and a four node. The purpose of both types of MPCs is to remove the degrees of freedom of the first node by constraining them to another node at the same coordinates. As stated above, the list order of the MPC is crucial to the proper enforcement of the constraints. A sample of the input for the MPCs of the structure of Fig. 1.2 is the following.

$\begin{array}{rrrrr}1, & 3, & 2 & & \\ 2, & 5, & 4 & & \\ 1, & 8, & 9 & & \\ 4, & 9, & 15 & & \\ 6, & 15, & 14 & & \\ 3, & 14, & 8, & 9, & 15\end{array}$

Shown in Fig. 1.3, the first two MPCs listed above correspond to the exterior coincident nodes on the bottom of the structure. Shown in Fig. 1.4, the last four correspond to the lower left set of four coincident nodes. 


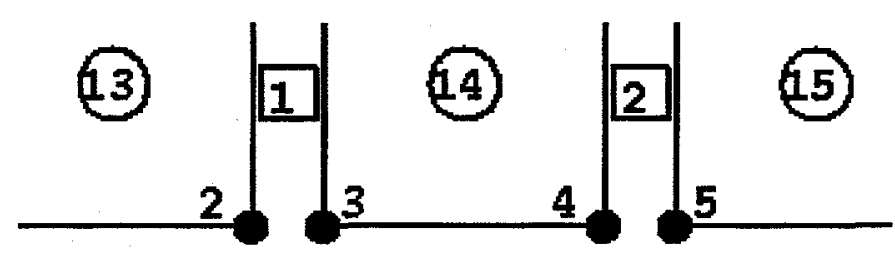

Figure 1.3. Bottom two sets of coincident nodes.

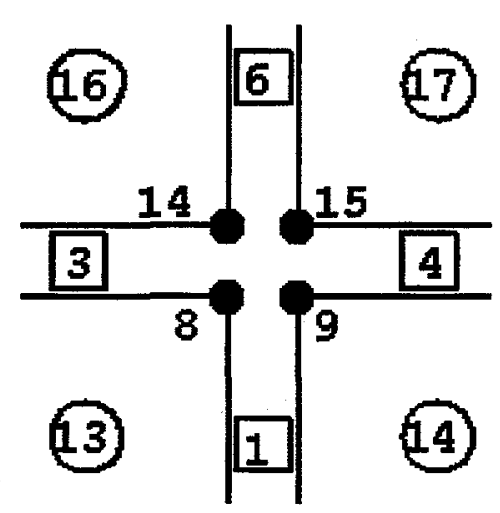

Figure 1.4. Lower left set of four coincident nodes.

The pertinent ABAQUS and user defined variables used in this subroutine, along with their dimensions, are listed below.

ABAQUS variables

$n=$ number of nodes involved in the constrain

jtype $=$ constraint identifier given on input data line

$l m p c=\mathrm{MPC}$ enforcement flag $(=0$, don't enforce MPC)

$n d e p=$ number of dependent degrees of freedom involved in the

constraint

$m d o f=$ number of active degrees of freedom per node

$u e($ ndep $)=$ total value of the eliminated degrees of freedom

$a(m d o f, m d o f, n)=$ submatrices of coefficients of linearized constraints

$j d o f(m d o f, n)=$ matrix of degree of freedom identifiers at the nodes

involved in the constraint

User defined variables

ifailf $(100000)=$ interface failure indicator

nodefail $(100000)=$ node failure indicator

node\# = node numbers of node involved in the constraint

\subsubsection{Two Node}

As discussed above, there are two different multi-point constraints. The simpler of these is a 2-node multi-point constraint. The purpose if the 2-node MPC is to replace the two translational degrees of freedom of the first node with the degrees of freedom of the second node (i.e. rigidly tie the first node to the second node). This is a simple task, the theory of which is discussed in the ABAQUS User's Manual. 
Also mentioned above, the order of the nodes in a single interior MPC and the order each interior MPC is listed are crucial to proper enforcement of the constraint. In addition, the number, or type, of each MPC must correspond to the ICE number. When failure of an ICE occurs, each MPC of that type is no longer enforced. This is accomplished via the ABAQUS variable lmpc. The value of this variable is set to 0 to turn off the MPC. Then, for each call to the MPC subroutine, if the type corresponds to a failed ICE, $l m p c$ is set to zero and no degrees of freedom are removed.

\subsubsection{Four Node}

The second type of MPC used in this work is the four node MPC. There is a four node MPC for each internal location with four coincident nodes. The purpose of this type of MPC is to ensure proper enforcement of the MPC after a macroscopic crack has grown through a four node location. The four node MPC is similar to the two node MPC. It is set up so that the four node MPC will remove the degrees of freedom of the first node by constraining them to the degrees of freedom of one of the other three nodes. Difficulty arises in determining which one of the other three nodes to attach it to.

The four node MPCs are only "active" when one or more ICEs have failed at a location. This is because all of the degrees of freedom of the three independent nodes have been removed by the previous two node MPCs. If an ICE fails at a location, the MPC corresponding to the interface nodes is no longer enforced. The dependent node of the failed MPC is no longer constrained to another node. The dependent node of the four node MPC is then constrained to this "free" node.

To accomplish this, a nodal failure flag, along with the ICE failure flag, must be assigned to determine which of the three independent nodes in the four node MPC is unconstrained. In the UEL subroutine, discussed later, when the failure criterion or criteria have been met for interface failure, the ICE failure flag for the failed ICE is set. In addition, a nodal failure flag for the first and fifth nodes on the ICE connectivity list is set. Recall the first and fifth nodes correspond to the first node for each adjacent solid element. Then for each call to the MPC subroutine for a four node MPC, if any of the failure flags for the three independent nodes have been set, the dependent node is attached to that node. If more than one nodal failure flag is set, the dependent node is attached to the first node with the failure flag set.

For example, the sample listing given previously in this section shows one four node MPC. The sample listing corresponds to the intersection of ICEs 1, 3, 4, and 6 in Fig. 1.2. The four node MPC is inactive until one of the ICEs 1, 3, 4, or 6, fails. Then if ICE 1 fails, the failure flag for ICE 1 and the nodal failure flag for nodes 3 and 8 are set. The two node MPC number 1 is no longer enforced. Hence, the degrees of freedom of node 8 are no longer removed from the problem. The dependent node of the four node MPC (node 14) is then constrained to node 8. This system works for any combination of single or multiple ICE failures. 


\subsubsection{User Element}

As used in this work, the user element subroutine provides the necessary structural and dynamic information for each element, determines if the solution has converged within reasonable tolerances, and evaluates the interface failure criteria. The ABAQUS and user defined variables used in this subroutine are listed below.

ABAQUS variables

$$
\begin{aligned}
& \text { dtime }=\text { time increment } \\
& \text { jelem }=\text { user assigned element number }
\end{aligned}
$$$$
\text { kinc }=\text { current increment number }
$$

pnewdt $=$ ratio of suggested new time increment to current time

increment

mlvarx $=$ dimension parameter for several right hand side vectors ndofel $=$ number of degrees of freedom in the element

nsvars $=$ number of solution dependent state variables

$m c r d=$ number of dimensional coordinates

nnode $=$ number of nodes for the element

$n d o f e l=$ number of degrees of freedom for the element

npredef $=$ number of predefined field variables

rhs $\left(\operatorname{mlvar} x{ }^{*}\right)=$ element contribution to the right hand side vector

amatrx (ndofel,ndofel) $=$ element contribution to the Jacobian matrix

$\operatorname{props}(*)=$ real element properties

svars $($ nsvars $)=$ state variables

coords $($ mcrd, nnode $)=$ nodal coordinates

$u($ ndofel $)=$ current estimate of nodal displacements

$d u($ mlvar $x, *)=$ increment in nodal displacement

$a($ ndofel $)=$ current estimate of nodal accelerations

params $(*)=$ parameters associated with Hilbert-Hughes time

integration scheme

predef $(2$, npredf, nnode $)=$ predefined field variables

lflags $(*)=$ flags to define current solution procedure

jprops $(*)=$ integer element properties

User defined variables

$u \# x, u \# y=\mathrm{x}$ and $\mathrm{y}$ displacements for 4 nodes of one solid element

$x \#, y \#=$ coordinates for 4 nodes of one solid element

$b x, b y=\mathrm{x}$ and $\mathrm{y}$ components of body force for one solid element

thk\# = nodal thicknesses for one solid element

node 1 , node $5=$ first and fifth nodes of ICE

$b=$ interface width

$h=$ element height

$d d u m=$ largest correction to increment of displacement from previous iteration

ddumo = previous value of ddum

$d u m=$ largest increment of displacement from previous iteration

$e m=$ Young's Modulus

$g n u, n u=$ Poisson's Ratio 
$g l c=$ crack growth resistance curve parameter

$a c=$ current interface crack half width

rho $=$ mass density

$g=$ Shear Modulus

sigult $=$ ultimate strength

iadj\# = two adjacent element numbers

iplnstrn,iplnstrss $=$ plane stress and plane strain flag

iequiliter $=$ count of how many times a single element has been called

during one increment

sig0, sig1, tau $0=$ far field stresses

$s x, s y, t x y=\mathrm{x}, \mathrm{y}$ and $\mathrm{xy}$ components of stress for one solid element

$e 1, e 2, e 12=\mathrm{x}, \mathrm{y}$ and $\mathrm{xy}$ components of strain for one solid element

$e b x x, e b y y, e b x y=$ average components of strain at interface

jfail $=$ convergence flag

ifli $=$ interface failure during last iteration flag

noel $=$ number of solid elements

ielno $=$ solid element number

numvfe $=$ number of ICEs

ivfeno $=$ ICE number

alpha, beta $=$ Hilber-Hughes time integration parameters

$d t=$ time increment

sum $=$ sum used for matrix multiplication

$x i$, eta $=$ local coordinates in isoparametric coordinate system for Gauss

Quadrature

$x i s i g n=\operatorname{sign}$ of $x i$

det $j=$ determinant of Jacobian matrix

thick $=$ interpolated thickness

$R N \#$ = element shape functions

$x, y=$ local coordinates in curvilinear coordinate system corresponding

to $x i$, eta

$i c=$ count of iteration following an interface failure

$p \# x, p \# y=$ current node locations

$v \# x, v \# y=$ components of vectors between nodes

$v n \# x, v n \# y=$ components of vector normal to vectors connecting nodes

$v t \# x, v t \# y=$ components of sum of normal vectors

$r \# x, r \# y=$ components of local $\mathrm{z}$ direction vector

$x x \#, y y \#=$ global coordinates of nodes with origin at center of ICE

interface

$g \#=$ factors in crack strain equations

$a a, b b, c c, a t f 1, a t f 2, c f, c f 1, c f 2=$ intermediate factors in $g \#$

exi\#, eeta\# = xi and eta components in crack strain equations

$s p a=\sqrt{\pi a}$

$r k \#=$ factors in gval

$k 1$ sign $=$ sign of sig0

gval $=$ interface strain energy release rate

rlamd $a=$ coefficient in crack growth resistance curve 
$m=$ exponent in crack growth resistance curve

$r l=$ offset in crack growth resistance curve

delta $a=$ current estimate of total interface crack growth

in = number of sampling points for averaging interface stresses and strains

- $t \#$ = factors in matrix assembly; from Maple V output

ivfeel $(100000,2)=$ adjacent solid element numbers for each ICE

thk $(100000,8)=$ nodal thickness for each solid element for each ICE

$b f(100000,2)=$ body force for each solid element for each ICE

$a 0(100000)=$ original interface crack length for each interface

$q(2,2), q t(2,2)=$ transformation matrix

$q e(2,2)=$ intermediate matrix in transformation

$q e q(2,2)=$ final step in transformation

$g p(4)=$ evaluation points for Gauss Quadrature

$g w(4)=$ weights for Gauss Quadrature

$e v(3)=$ integrated components of applied strain field

ifailf $(100000)=$ interface failure flag

nodefail $(100000)=$ nodal failure flag

istiff $(100000)=$ stiffness assignment flag

inewel( 100000$)=$ ICE evaluation flag

iold $(100000)=$ increment number; indicates new increment

st $(9,3)=$ transpose of $\mathbf{S}$; from formulation

$G A H A(8,9)=$ coefficient in load vector; from formulation

$t x(4), t y(4)=$ local node coordinates

$d u o(100000,16)=$ increment of displacement from previous iteration

for each ICE

epsilon $(100000,3)=3$ components of strain for each solid element

$\operatorname{eps}(3)=3$ components of strain for on solid element

$e b(2,2)=$ matrix of tensor state components

evfe $(3)=$ components of externally applied strain field

sigma(3) $=3$ components of stress for one solid element

$\operatorname{sig}(3)=3$ components of stress for one solid element

$\operatorname{sxx}(2)=\mathrm{x}$ stress for both solid elements

$s x y(2)=y$ stress for both solid elements

$s y y(2)=x y$ stress for both solid elements

$\operatorname{rmsmt}(8,8)=$ mass matrix for one solid element

$r m s m t 2(16,16)=$ mass matrix for both solid elements of one ICE

rmasmat $(16,16)=$ mass matrix for one ICE

$r m(8,8)=$ mass matrix for one solid element

massflag4(50000) = mass matrix assignment flag for velocity jump

calculation

massflag6 $(50000)=$ mass matrix assignment flag for initial acceleration

calculation

$r k(8,8)=$ stiffness matrix for one solid element

rksave $(16,16)=$ stiffness matrix for both solid elements of one ICE

$g(16,100000)=$ current $\mathbf{K}^{*} \mathbf{u}$ for rhs vector for each ICE 
$\operatorname{gold}(16,100000)=$ previous increment $\mathbf{K} * \mathbf{u}$ for each ICE

ftop $(8)=$ crack strain component of rhs vector for one solid element

ftop $2(16,100000)=$ crack strain component of rhs vector for both solid elements for each ICE

ftopold $(16,100000)=$ crack strain component of rhs vector for previous increment for each ICE

$f 3(8)=$ body force component of rhs vector for one solid element

\subsubsection{First Entry}

Upon the first entry into the UEL subroutine, the information from the external files is read and stored in arrays. The three files body, thick, and vfedeck are opened. The $\mathrm{x}$ and $\mathrm{y}$ body forces for each solid element are read into the array $b f($ element no., direction). The thickness for each node of each solid element is read into the array thk(element no.,1-4). The adjacent solid elements for each ICE is read into the array ivfeel(ICE no., 1-2). The initial interface crack half width for each ICE is read into the array $a 0$ (ICE no.).

\subsubsection{Procedure Type}

The UEL subroutine must support several different procedures for a dynamic analysis. The third element in the lflags array contains the necessary information to determine which procedure ABAQUS is performing. The information that must be passed back to ABAQUS can then be determined.

At the beginning of an analysis step, ABAQUS determines the velocity jump and initial acceleration. For these two procedures, lflags $(3)=4$ and lflags $(3)=6$, respectively. For each of these two procedures, the mass matrix for both adjacent solid elements is assembled into the amatrx array, if it mass has not been previously assigned, and returned to ABAQUS. The load vector ( $r h s$ ) is returned unchanged (every element remains zero). These are the only operations performed for each of these two procedures.

The automatic time stepping algorithm requires the calculation of a half-step residual. That is, a residual half way in between time steps. For this procedure, Iflags $(3)=5$. In this case, the time step that is passed into the subroutine, dtime, is divided by two and the standard dynamic procedure is used to return the element Jacobian matrix and load vector. During the half-step residual procedure; a) solution convergence is not checked, b) interface stress and strain states are not calculated, and c) interface crack growth and failure check is not performed. Because the interface stress and strain states are not calculated for the half-step residual, the accuracy of this calculation is in question. This is of little concern because the half-step residual does not affect the solution and the automatic time step selection based on the half-step residual has been effectively disabled.

For lflags $(3)=1$, a "standard" dynamic analysis is being performed. The UEL subroutine returns the standard dynamic Jacobian matrix (amatrx) and load vector $(r h s)$. Note that during the first time increment, the load vector is the standard load 
vector for a plane element and does not include the effects of the interface cracks. This is due to the values of the displacement vector, $u$, being zero upon the first iteration of the first time increment. This causes mathematical errors in the crack strain equations. Hence, the load vector for the interface cracks is not assembled until the second time increment.

\subsubsection{Subroutine ENTER}

Upon each entry into the UEL subroutine, the subroutine ENTER is called to perform specific initial computations. Adjacent element numbers, material properties, plane stress or plane strain conditions, interface crack size and other element information that is stored in various arrays is assigned to intuitive variables. Also, initial failure of interfaces is checked ${ }^{9}$ and the necessary failure flags set.

It is assumed that the UEL subroutine is called once for each ICE for each iteration. Hence, after each ICE has been passed into the UEL subroutine, the iteration is complete and a new iteration begins. The entry flag array, inewel, corresponding to each ICE is set (equal to 1) upon each call. Then, a new iteration is determined when the entry flag for any ICE has been set prior to entry into the UEL subroutine ${ }^{10}$. Then, if it is determined that it is a new iteration:

1. The stiffness assignment flag array, istiff, is reset,

2. The entry flag array, inewel, is reset,

3. Convergence of the solution in the last iteration is checked,

4. The increment in displacement from the last iteration is saved,

5. If the time increment has changed from the previous iteration, the equilibrium iteration counter, iequiliter, is reset.

Several items must be checked in order to determine if the solution converged in the previous iteration. Because ABAQUS performs steps that are intermediate to the solution iterations, convergence must be checked for each iteration step and not each intermediate step. The two variables used to determine convergence are the maximum increment of displacement, dum, and the maximum change in increment of displacement, ddum. Then, for an exact solution, ddum would be zero. The primary convergence parameter is the ratio ddum/dum. If this is sufficiently small, it is likely that the solution is relatively accurate.

A common feature of the intermediate solution steps is the change in increment of displacement, ddum, is significantly smaller than on solution iterations. This presents a problem in that it can be falsely determined that the solution has converged based on these intermediate steps. To help remove these problems the following

\footnotetext{
${ }^{9}$ Initial failure is accomplished by setting the initial crack length wider than the interface. This is done for ease of modeling the original geometry. For instance, an initial sharp crack is easily modeled using this method.

${ }^{10}$ This is not correct. ABAQUS performs intermediate steps in which each ICE is passed in to UEL. These steps are not solution iterations. A reliable method for distinguishing between iterations and intermediate solution steps has not been devised. This causes difficulties in determining solution convergence, but all other computations are valid and correct.
} 
convergence checks are made:

1. $d d u m / d d u m o<s v$
2. $d u m<s v$
3. $d d u m<s v$

Where ddumo is the "old" or previous value of ddum and $s v$ is a small value that depends on the specific problem being analyzed. Then if any of the above are true, it is assumed the procedure is an intermediate step and the solution has not converged.

In addition to the above convergence criteria the following are also used:

1. if either dum or ddum is large relative to the specific problem, it is assumed the current solution is far from the correct solution and convergence has not been reached,

2. if the value of $d d u m$ has increased from the previous increment, the solution has not converged,

3. if the user count of iterations, iequiliter, is below a specified value, the solution procedure is premature has not converged,

4. if any interface failed in the previous iteration, the solution has not converged,

5. if the time increment has changed from the previous iteration, the solution has not converged.

After solution convergence is checked, it is then determined if the call to UEL is for a new time increment. If this is the case:

1. The load vector components, ftop 2 and $g$, from the previous time increment are saved in the arrays ftopold and gold.

2. The equilibrium iteration counter is reset.

\subsubsection{Interface Stress State and Failure}

After the preliminary computations have been done in the ENTER subroutine for each call to UEL, the stress and strain states of the interface are determined. Then, from the calculated stress state, the interface crack growth is determined and the gross stress failure criterion is evaluated.

The stress and strain at any point in an isoparamteric element is assembled and integrated symbolically in Maple V. The subroutines to determine element stress and strain are STRSS and STRN respectively. The output from Maple V calculates the element stress and strain for any value of the isoparametric coordinates, (xi,eta). The two subroutines evaluate the stress and strain at the edge of a solid element as shown in Fig 1.5. The values are then averaged to determine the average stress and strain components along the edge of the element corresponding to the ICE interface.

The far-field stresses, sigO, sigl, and tauO, are determined from the averaged interface stress state. In the subroutine SIF, the strain energy release rate, gval, at the interface is calculated from the far-field stresses. In the subroutine AUPDATE, the 


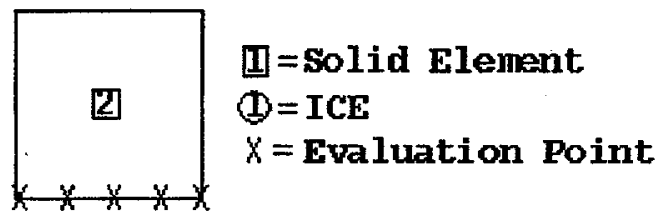

(1)

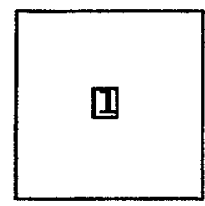

Figure 1.5. Evaluation point for the averaging stress and strain along an ICE interface.

change in crack length, deltaa, is calculated using the local strain energy release rate and the inverted crack growth resistance curve.

The gross stress failure criterion is evaluated by comparing the far-field normal stress, sig0, to the ultimate stress, sigult. If the normal interface stress exceeds the ultimate stress, the interface crack width is set to a large value. Gross shear failure is not included at this time, but it could be added easily.

These values of interface crack length and failure correspond to the current estimate to the solution. No check of solution convergence is performed here. The complete failure of an interface based on the crack length and solution convergence is checked at a later point in the UEL subroutine (discussed in section 1.2.2.6).

\subsubsection{Structural Contributions}

The structural contributions for each ICE are assembled into the Jacobian matrix and load vector. The load vector assembled here is for a standard plane element without the applied crack strain load vector. The arrays for the two 4 node solid elements are constructed separately and then assembled into the amatrx and rhs arrays for the 8 node user element.

Upon entry into this section of the UEL subroutine, the user defined arrays used to store the solid element and ICE stiffness and mass matrices are set to zero. This is done to ensure that stiffness and mass contributions of a solid element are zero if they have been defined by a previous ICE. The assignment of stiffness, and therefore mass, during a previous call to the UEL subroutine is determined by the istiff flag array. When the stiffness and mass are assigned to a solid element, the corresponding flag in istiff is set to 1 . Then, if the flag has been set upon entry into UEL, no stiffness and mass are assigned to that solid element.

The mass matrix and stiffness matrix for a single solid element are construct by calling the subroutines MM and HWE. The HWE subroutine also returns the standard body force load vector. If the stiffness flag indicates that mass and stiffness has not been assign to the current solid element, the elements in amatrx corresponding to the 4 nodes of the solid element are assembled, otherwise the corresponding elements of amatrx are not changed and remain zero. The structural load vector corresponding to 
a 4 node solid element is then assembled using the (zero or true) mass and stiffness. This process is then repeated for the second adjacent solid element.

\subsubsection{Interface Failure}

If the interface crack is wider than the interface, due to crack growth or gross stress failure, and the interface has not failed previously, the failure of the interface is evaluated. Before an interface is allowed to fail, two criteria must be met.

1. First, the solution must be within the defined convergence tolerances as determined in subroutine ENTER.

2. Second, the time step must be below the user defined value. If the solution has converged and the time step is above the user defined value, the value of pnewdt is set to a value less than 1 and the interface is not allowed to fail.

If both of these criteria are met and the crack growth or gross stress failure criteria have been met, the interface fails and the corresponding element in the interface failure flag array, ifailf, and node failure flag array, nodefail, are set.

Also in this section of the UEL subroutine, the maximum increment of displacement, dum, and maximum change in displacement increment, ddum, are determined. This is done by comparing the maximums for the values of the nodes of the current ICE with the previous maximums for this iteration.

\subsubsection{Interface Crack Contributions}

If there is an interface crack that has not failed, a new load vector is constructed for each adjacent solid element and assembled into the rhs load vector. The average "far-field" strains ${ }^{11}$ are calculated by averaging the interface strains of the two neighboring elements and assigned to the variables $e b x x$, ebyy and $e b x y$. These strains are used to determine the strain field due only to the presence of a crack at the interface.

The subroutine VFE assembles the crack strain component of the load vector, ftop, for a single solid elements. The subroutine VFE assembles the load vector component by numerically integrating the crack strain components and multiplying by the symbolically assembled matrix coefficients. The quantity that is numerically integrated is the following

$$
\text { evfe }{ }^{*} \text { detj }{ }^{*} \text { thick }
$$

where evfe is an array of the components of the applied strain field, detj is the determinant of the mapping matrix from isoparametric to curvilinear coordinates, and thick is the interpolated thickness. The equation above must be multiplied by the appropriate weight factor, $g w($ ), and summed over all the evaluation points, $g p()$.

Because the crack strains do not scale, this must be done for the curvilinear element, not the isoparametric element. To do this, a standard right-handed coordinate system is defined at the center of the interface with positive y being normal to the interface in the direction of the solid element, as shown in Fig. 1.6. This translation of

\footnotetext{
${ }^{11}$ The strains for a standard plane element based on nodal displacements. Comparable to the far-field stress discussed earlier, the strains are averaged at the ICE interface.
} 
coordinates and the local coordinates of each node, $(t x, t y)$, are obtained from the subroutine TRANSFORMXY. The evaluation points for Gauss Quadrature are then mapped into the element. At each of these mapped points the equation above is evaluated; a) the interpolated thickness is calculated from a simple equation, b) the components of the applied strain field at the mapped point are obtained from the subroutine EVFE, and c) the determinant of the mapping matrix is obtained from the subroutine DETJ.

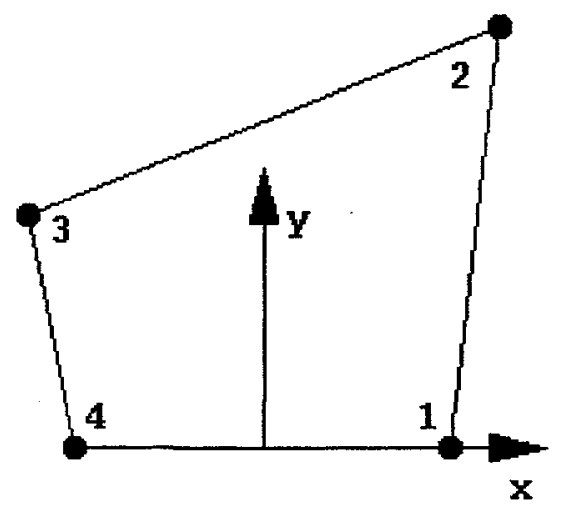

Figure 1.6. Local coordinate system for numerical integration of crack strain equations.

The numerically integrated components of the crack strain are then multiplied by the symbolically integrated coefficient matrices. The crack strain component of the load vector is then assembled into the element load vector, rhs. This overwrites any previous values that have been assembled into the load vector. 


\section{Files}




\title{
2.1 ABAQUS Input File
}

\begin{abstract}
*HEADING
UEL Problem

*NODE, NSET $=$ ALL

$1,0.000000000 \mathrm{E}+00,0.000000000 \mathrm{E}+00,0.000000000 \mathrm{E}+00$

$2,0.333333343 \mathrm{E}+00,0.000000000 \mathrm{E}+00,0.000000000 \mathrm{E}+00$

$3,0.333333343 \mathrm{E}+00,0.000000000 \mathrm{E}+00,0.000000000 \mathrm{E}+00$

$4,0.666666687 \mathrm{E}+00,0.000000000 \mathrm{E}+00,0.000000000 \mathrm{E}+00$

$5,0.666666687 \mathrm{E}+00,0.000000000 \mathrm{E}+00,0.000000000 \mathrm{E}+00$

$6,0.100000000 \mathrm{E}+01,0.000000000 \mathrm{E}+00,0.000000000 \mathrm{E}+00$

$7,0.000000000 \mathrm{E}+00,0.333333343 \mathrm{E}+00,0.000000000 \mathrm{E}+00$

$8,0.333333343 \mathrm{E}+00,0.333333343 \mathrm{E}+00,0.000000000 \mathrm{E}+00$

$9,0.333333343 \mathrm{E}+00,0.333333343 \mathrm{E}+00,0.000000000 \mathrm{E}+00$

$10,0.666666687 \mathrm{E}+00,0.333333343 \mathrm{E}+00,0.000000000 \mathrm{E}+00$

$11,0.666666687 \mathrm{E}+00,0.333333343 \mathrm{E}+00,0.000000000 \mathrm{E}+00$

$12,0.100000000 \mathrm{E}+01,0.333333343 \mathrm{E}+00,0.000000000 \mathrm{E}+00$

$13,0.000000000 \mathrm{E}+00,0.333333343 \mathrm{E}+00,0.000000000 \mathrm{E}+00$

$14,0.333333343 E+00,0.333333343 E+00,0.000000000 E+00$

$15,0.333333343 \mathrm{E}+00,0.333333343 \mathrm{E}+00,0.000000000 \mathrm{E}+00$ $16,0.666666687 \mathrm{E}+00,0.333333343 \mathrm{E}+00,0.000000000 \mathrm{E}+00$ $17,0.666666687 \mathrm{E}+00,0.333333343 \mathrm{E}+00,0.000000000 \mathrm{E}+00$ $18,0.100000000 \mathrm{E}+01,0.333333343 \mathrm{E}+00,0.000000000 \mathrm{E}+00$ $19,0.000000000 \mathrm{E}+00,0.666666687 \mathrm{E}+00,0.000000000 \mathrm{E}+00$ $20,0.333333343 \mathrm{E}+00,0.666666687 \mathrm{E}+00,0.000000000 \mathrm{E}+00$ $21,0.333333343 \mathrm{E}+00,0.666666687 \mathrm{E}+00,0.000000000 \mathrm{E}+00$ $22,0.666666687 \mathrm{E}+00,0.666666687 \mathrm{E}+00,0.000000000 \mathrm{E}+00$ $23,0.666666687 \mathrm{E}+00,0.666666687 \mathrm{E}+00,0.000000000 \mathrm{E}+00$ $24,0.100000000 \mathrm{E}+01,0.666666687 \mathrm{E}+00,0.000000000 \mathrm{E}+00$ $25,0.000000000 \mathrm{E}+00,0.666666687 \mathrm{E}+00,0.000000000 \mathrm{E}+00$ $26,0.333333343 \mathrm{E}+00,0.666666687 \mathrm{E}+00,0.000000000 \mathrm{E}+00$ $27,0.333333343 \mathrm{E}+00,0.666666687 \mathrm{E}+00,0.000000000 \mathrm{E}+00$ $28,0.666666687 \mathrm{E}+00,0.666666687 \mathrm{E}+00,0.000000000 \mathrm{E}+00$ $29,0.666666687 E+00,0.666666687 E+00,0.000000000 E+00$ $30,0.100000000 \mathrm{E}+01,0.666666687 \mathrm{E}+00,0.000000000 \mathrm{E}+00$ $31,0.000000000 \mathrm{E}+00,0.100000000 \mathrm{E}+01,0.000000000 \mathrm{E}+00$ $32,0.333333343 \mathrm{E}+00,0.100000000 \mathrm{E}+01,0.000000000 \mathrm{E}+00$ $33,0.333333343 \mathrm{E}+00,0.100000000 \mathrm{E}+01,0.000000000 \mathrm{E}+00$ $34,0.666666687 \mathrm{E}+00,0.100000000 \mathrm{E}+01,0.000000000 \mathrm{E}+00$ $35,0.666666687 \mathrm{E}+00,0.100000000 \mathrm{E}+01,0.000000000 \mathrm{E}+00$ $36,0.100000000 \mathrm{E}+01,0.100000000 \mathrm{E}+01,0.000000000 \mathrm{E}+00$ *ELEMENT , TYPE=CPS4, ELSET $=a b e 101$
\end{abstract}

$\begin{array}{rrrrr}13, & 2, & 8, & 7, & 1 \\ 14, & 4, & 10, & 9, & 3 \\ 15, & 6, & 12, & 11, & 5 \\ 16, & 14, & 20, & 19, & 13 \\ 17, & 16, & 22, & 21, & 15 \\ 18, & 18, & 24, & 23, & 17 \\ 19, & 26, & 32, & 31, & 25 \\ 20, & 28, & 34, & 33, & 27 \\ 21, & 30, & 36, & 35, & 29\end{array}$

*SOLID SECTION, ELSET=abe101, MATERIAL=steel01

$.10000 \mathrm{E}-09$

*MATERIAL , NAME=steel 01

*ELASTIC

$.10000 \mathrm{E}-03, .30000 \mathrm{E}+00$, 
*DENSITY

$.10000 \mathrm{E}-09$

*USER ELEMENT,

TYPE $=U 1$, NODES $=8$, COORDINATES $=2$, PROPERTIES $=5$, VARIABLES $=23$, IPROPERTIES $=2$

1,2

*ELEMENT, TYPE $=U 1$, ELSET $=v f e 101$

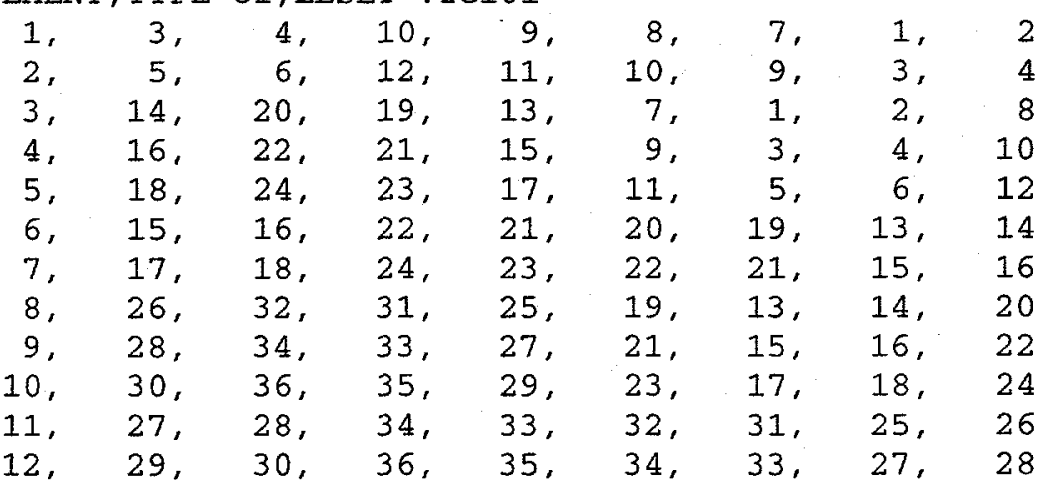

*UEL PROPERTY, ELSET $=v$ fel01

$.30000 \mathrm{E}+08, .30000 \mathrm{E}+00, .21300 \mathrm{E}+03, .73000 \mathrm{E}-03, .10000 \mathrm{E}+06$,

*MPC, USER, MODE $=\mathrm{NODE}$

\begin{tabular}{|c|c|c|c|c|}
\hline 1, & 3 & 2 & & \\
\hline 2 , & 5 & 4 & & \\
\hline 3, & 7, & 13 & & \\
\hline 1, & 8, & 9 & & \\
\hline 4, & 9 & 15 & & \\
\hline 6 & 15. & 14 & & \\
\hline 3, & 14, & 8 & 9 , & 15 \\
\hline 2 & 10 & 11 & & \\
\hline 5, & 11, & 17 & & \\
\hline 7, & 17. & 16 & & \\
\hline 4, & 16, & 10, & 11, & 17 \\
\hline 5, & 18, & 12 & & \\
\hline 8, & 19. & 25 & & \\
\hline 6, & 20. & 21 & & \\
\hline 9, & 21 & 27 & & \\
\hline 11, & 27 & 26 & & \\
\hline 8 & 26 & 20 & 21, & 27 \\
\hline 7 & 22 & 23 & & \\
\hline 10, & 23. & 29 & & \\
\hline 12 , & 29, & 28 & & \\
\hline 9 & 28, & 22, & 23, & 29 \\
\hline 10. & 30, & 24 & & \\
\hline 11, & 32, & 33 & & \\
\hline 12 , & 34, & 35 & & \\
\hline
\end{tabular}

*BOUNDARY

$\begin{array}{lll}1, & 1, & 2, \\ 2, & 1, & 2, \\ 3, & 1, & 2, \\ 4, & 1, & 2, \\ 5, & 1, & 2, \\ 6, & 1, & 2,\end{array}$

*AMPLITUDE , NAME=AMP1, DEFINITION=EQUALLY SPACED , FIXEDINTERVAL $=.100 \mathrm{E}+01$

$.0000 \mathrm{E}+00, .1000 \mathrm{E}+01$

*RESTART, WRITE

*STEP, INC $=100$, MONOTONIC $=$ NO, UNSYMM $=$ YES

$\star$ DYNAMIC , HAFTOL $=1 . e 36$, ALPHA $=-0.00$ 


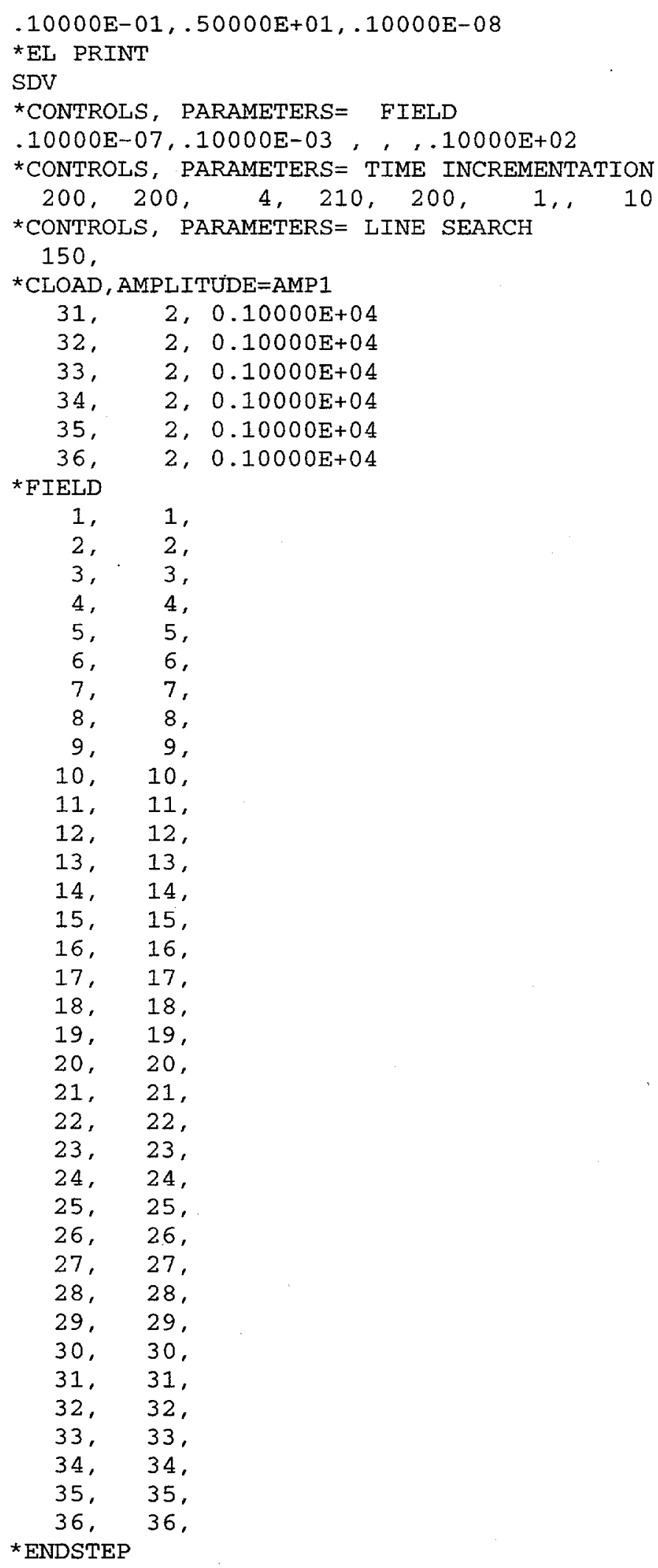




\subsection{Maple Worksheet}

This is a list of the input commands to Maple $V$ used to produce the arrays in the UEL Subroutine. Execution of this entire worksheet, excluding the comments, will reproduce the removed portions of the subroutine. Both the plane stress and plane strain arrays are formulated by the choice of the elastic moduli array ED; (1) plane stress, (2) plane strain. The command for FORTRAN output for each array is denominated by a letter and referenced in the UEL listing at the location in which the output has been removed.

$>$ with $($ linalg):

$>\mathrm{N} 1:=1 / 4 *(1-\mathrm{xi}) *(1-\mathrm{eta}) ; \mathrm{N} 2:=1 / 4 *(1+\mathrm{xi}) *(1-\mathrm{eta}) ; \mathrm{N} 3:=1 / 4 *(1+\mathrm{xi}) *(1+$ eta $) ; \mathrm{N} 4:=1 / 4 *(1-\mathrm{xi}) *(1+$ eta $) ;$

$>\mathrm{N}:=\operatorname{matrix}(4,1,[\mathrm{~N} 1, \mathrm{~N} 2 ; \mathrm{N} 3, \mathrm{~N} 4])$;

$>\mathrm{x}:=\mathrm{N} 1 * \mathrm{x} 1+\mathrm{N} 2 * \mathrm{x} 2+\mathrm{N} 3 * \mathrm{x} 3+\mathrm{N} 4 * \mathrm{x} 4 ; \mathrm{Y}:=\mathrm{N} 1 * \mathrm{y} 1+\mathrm{N} 2 * \mathrm{y} 2+\mathrm{N} 3 * \mathrm{y} 3+\mathrm{N} 4 * \mathrm{y} 4$

$>\mathrm{J}:=\operatorname{matrix}(2,2,[\operatorname{diff}(\mathrm{x}, \mathrm{xi}), \operatorname{diff}(\mathrm{y}, \mathrm{xi}), \operatorname{diff}(\mathrm{x}$, eta $), \operatorname{diff}(\mathrm{y}, \mathrm{eta})])$;

$>\operatorname{det} j:=\operatorname{det}(J)$;

$>$ fortran (detj, precision $=$ double, filename $=$ dj $)$;

$>$ pN1xy:=evalm(inverse(J) \&* (matrix $(2,1,[\operatorname{diff}(\mathrm{N} 1, \mathrm{xi}), \operatorname{diff}(\mathrm{N} 1, \mathrm{eta})]))) ; \mathrm{pN1x}:=\mathrm{pN1xy}[1,1] ; \mathrm{pN1y}:=\mathrm{pN1xy}[2,1] ;$

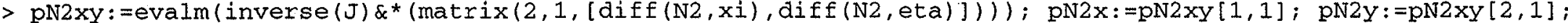

$>$ pN3xy:=evalm(inverse(J) \&* (matrix $(2,1,[\operatorname{diff}(\mathrm{N} 3, \mathrm{xi}), \operatorname{diff}(\mathrm{N} 3, \mathrm{eta})]))) ; \operatorname{pN} 3 \mathrm{x}:=\mathrm{pN} 3 \mathrm{xy}[1,1] ; \operatorname{pN} 3 \mathrm{y}:=\mathrm{pN} 3 \mathrm{xy}[2,1]$;

$>$ pN4xy: =evalm(inverse (J)\&*(matrix $(2,1,[\operatorname{diff}(\mathrm{N} 4, \mathrm{xi}), \operatorname{diff}(\mathrm{N} 4, \mathrm{eta})]))) ; \operatorname{pN} 4 \mathrm{x}:=\mathrm{pN} 4 \mathrm{xy}[1,1] ; \mathrm{pN} 4 \mathrm{y}:=\mathrm{pN} 4 \mathrm{xy}[2,1] ;$

$>\mathrm{B}:=\operatorname{array}(1 \ldots 3,1 \ldots 8)$;

$>$ entermatrix $(B)$;

$>$ pN1x;

$>0$;

$>\mathrm{pN} 2 \mathrm{x}$;

$>0 ;$

$>$ pN3xi

$>0$;

$>\mathrm{pN} 4 \mathrm{x}$;

$>0_{i}$

$>0 ;$

$>$ pN1y;

$>0$;

$>$ pN2y;

$>0$;

$>$ pN3y;

$>0$;

$>$ pN4Y;

$>$ pN1y;

$>$ pN1x;

$>$ pN2y;

$>$ pN2x;

$>$ pN3y;

$>$ pN3x;

$>$ pN4y; 
$>\mathrm{pN} 4 \mathrm{x} ;$

$>\operatorname{thk}:=\operatorname{matrix}(1,4,[\operatorname{thk} 1, \operatorname{thk} 2, \operatorname{thk} 3, \operatorname{thk} 4])$;

$>$ thickness:=evalm $($ thk $\& * N)$;

$>$ fortran (thickness, precision=double, filename=thick);

$>\mathrm{n}:=\operatorname{matrix}(2,8,[\mathrm{~N} 1,0, \mathrm{~N} 2,0, \mathrm{~N} 3,0, \mathrm{~N} 4,0,0, \mathrm{~N} 1,0, \mathrm{~N} 2,0, \mathrm{~N} 3,0, \mathrm{~N} 4])$;

$>$ bf: $=\operatorname{matrix}(2,1,[\mathrm{Bx}, \mathrm{By}])$;

$>f 3:=\operatorname{evalm}(\operatorname{transpose}(n) \& * b f * \operatorname{thickness}[1,1] * \operatorname{det} j)$;

$>\mathrm{F} 3:=\operatorname{array}(1 \ldots 8,1 \ldots 1)$;

$>$ for $i$ from 1 to 8 by 1 do for $j$ from 1 to 1 by 1 do $F 3[i, j]:=i n t(i n t(f 3[i, j], x i=-1 . .1)$, eta=-1..1) od od;

(a) Body force load vector

$>$ fortran (F3, precision=double, filename=body);

$>S:=\operatorname{matrix}(3,9,[1, x i$, eta $, 0,0,0,0,0,0,0,0,0,1$, xi, eta, $0,0,0,0,0,0,0,0,0,1, x i$, eta $])$;

$>$ si:=evalm(transpose $(s) * \operatorname{det} j * \operatorname{thickness}[1,1])$

$>\mathrm{ST}:=\operatorname{array}(1 \ldots 9,1 \ldots 3)$;

$>$ for $i$ from 1 to 9 by 1 do for $j$ from 1 to 3 by 1 do $\operatorname{ST}[i, j]:=i n t(i n t(\operatorname{si}[i, j], x i=-1 . .1)$, eta=-1..1) od od;

(b) transpose (S)*det (J)

$>$ fortran (ST, optimized,precision=double, filenamestj) :

$>\mathrm{E}:=\mathrm{S}$;

(1) Elastic Moduli Matrix for Plane stress

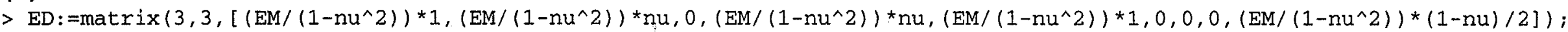
(2) Elastic Moduli Matrix for Plane strain

$>\operatorname{ED}:=(\operatorname{EM} *(1-n u) /((1+n u) *(1-2 * n u))) * \operatorname{matrix}(3,3,[1, \mathrm{nu} /(1-n u), 0, \mathrm{nu} /(1-n u), 1,0,0,0,(1-2 * n u) /(2 *(1-n u))]) ;$

$>\mathrm{u}:=\operatorname{matrix}(8,1,[\mathrm{u} 1 \mathrm{x}, \mathrm{u} 1 \mathrm{y}, \mathrm{u} 2 \mathrm{x}, \mathrm{u} 2 \mathrm{y}, \mathrm{u} 3 \mathrm{x}, \mathrm{u} 3 \mathrm{y}, \mathrm{u} 4 \mathrm{x}, \mathrm{u} 4 \mathrm{y}])$;

$>$ epsilon: =evalm $\left(B \&{ }^{*} u\right)$

\section{(c) element strain}

$>$ fortran (epsilon, optimized,precision=double, filename=eps) ;

$>$ sigma: $=$ evalm (ED\&*B\&*u);

\section{(d) element stress}

$>$ fortran (sigma, optimized, precision=double, filename=sigma):

$>\mathrm{g}:=\operatorname{evalm}(\operatorname{transpose}(S) \& * \mathrm{~B} * \operatorname{det}(\mathrm{J}) * \operatorname{thickness}[1,1])$ :

$>a:=\operatorname{evalm}\left(\right.$ transpose $\left.(S) \& * E^{*} \operatorname{det}(J) * \operatorname{thickness}[1,1]\right)$ :

$>\mathrm{h}:=$ evalm (transpose $(\mathrm{E}) \& * E D \& * \mathrm{E}^{*} \operatorname{det}(\mathrm{J}) *$ thickness $\left.[1,1]\right)$ :

$>A:=\operatorname{array}(1 \ldots 9,1 \ldots 9) ; G:=\operatorname{array}(1 \ldots 9,1 \ldots 8) ; \mathrm{H}:=\operatorname{array}(1 \ldots 9,1 \ldots 9) ;$

$>$ for $i$ from 1 to 9 by 1 do for $j$ from 1 to 9 by 1 do $A[i, j]:=i n t(i n t(a[i, j], \times i=-1,1)$, eta=-1..1) od od,

$>$ for $i$ from 1 to 9 by 1 do for $j$ from 1 to 9 by 1 do $H[i, j]:=i n t(i n t(h[i, j], x i=-1 \ldots 1)$, eta=-1..1) od od;

$>$ for $i$ from 1 to 9 by 1 do for $j$ from 1 to 8 by 1 do $G[i, j]:=i n t(i n t(g[i, j], x i=-1 \ldots 1)$, eta=-1..1) od odi

$>$ AK: $=\operatorname{array}($ symmetric, sparse, $1 \ldots 9,1 \ldots 9)$;

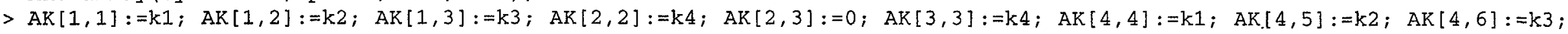

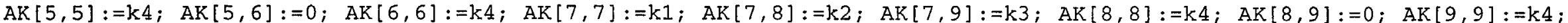

$>$ AKI : $=$ inverse $(\mathrm{AK})$

$>\operatorname{eval}(\mathrm{A})$;

$>\mathrm{k} 1:=81 ; \mathrm{k} 2:=82 ; \mathrm{k} 3:=83 ; \mathrm{k} 4:=84 ; \mathrm{k} 5:=0 ; \mathrm{k} 6:=84 ;$

$>$ GAHA : =evalm (transpose $(G) \& *$ transpose (AKI)\&*H\&*AKI);

(e) Coefficient in Crack strain Load Vector

$>$ fortran (GAHA, optimized,precision=double, filename=GAHA) ; 
$>r k:=\operatorname{evalm}\left(\right.$ transpose $\left.(G) \& * \operatorname{transpose}(A K I) \& * H \&{ }^{*} A K I \&{ }^{*}\right)$ :

(f) stiffness Matrix

$>$ fortran (rk, optimized,precision=double, filename=rk) ;

$>m:=\operatorname{evalm}\left(\right.$ transpose $(\mathrm{N}) \&{ }^{*} \mathrm{~N}^{*}$ thickness $\left.[1,1] * \operatorname{det}(\mathrm{J})\right)$;

$>\mathrm{M}:=\operatorname{matrix}(8,8)$;

$>$ for $i$ from 1 to 8 by 1 do for $j$ from 1 to 8 by 1 do $M[i, j]:=i n t(i n t(m[i, j], x i=-1 . .1)$, eta=-1..1) od od; (g) Mass Matrix

$>$ fortran (M, optimized,precision=double, filename=mass) ; 
2.3 Multi-Point Constraint Subroutine

This ABAQUS subroutine is described in Section 1.2.1 of this Appendix.

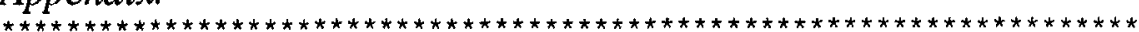
$* * *$

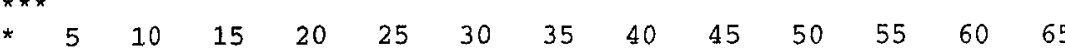

702

SUBROUTINE MPC (UE, A, JDOF, MDOF, N, JTYPE, X, U, UINIT MAXDOF, LMPC

* KSTEP, KINC, TIME, NT, NF, TEMP, FIELD, LTRAN, TRAN)

include 'ABA PARAM. INC'

dimension ue (mdof), $a$ (mdof, mdof, $n)$, jdof (mdof, $n), x(6, n)$

* u(maxdof,n), uinit (maxdof,n), time $(2), \operatorname{temp}(n t, n)$, field $(n f, n t, n)$ LTRAN (N), TRAN $(3,3, N)$

common/fail/ifailf(100000), nodefail(100000),jfail, ifli common/iter/iequiliter

C If the interface has failed, don't enforce the MPC

if (ifailf (jtype) eq. 1 ) then
lmpc=0
return
endif

C MPC to tie the two nodes given in the input

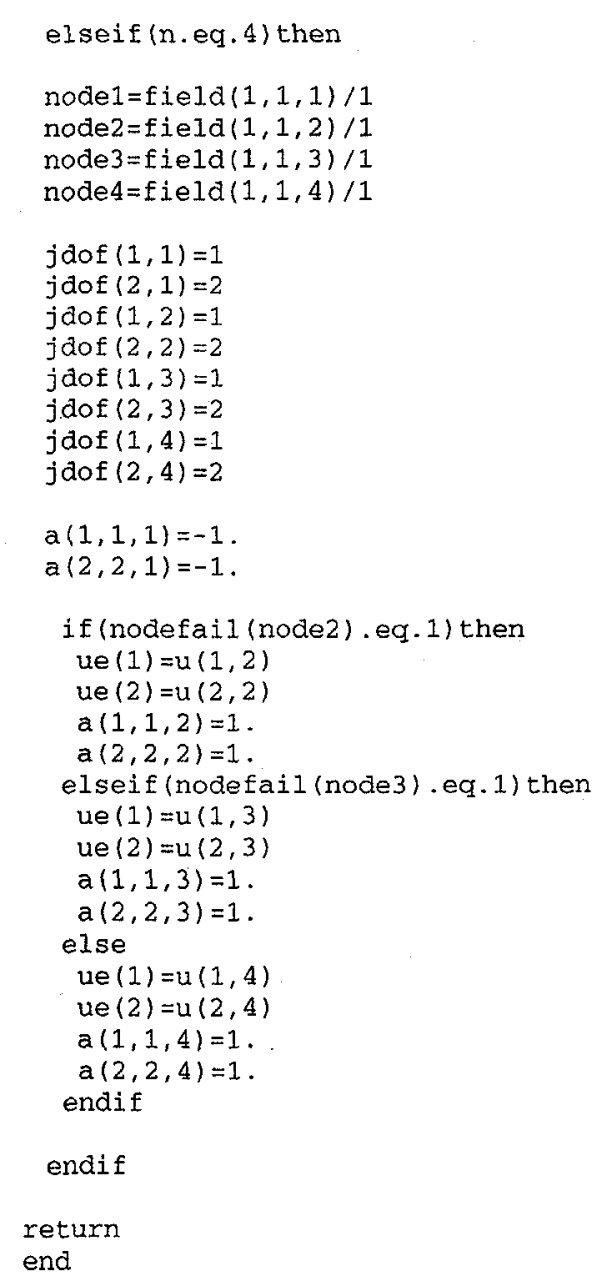


2.4 Abbreviated User Element Subroutine

This ABAQUS subroutine is described in Section 1.2.2 of this Appendix. A large part of the information in this subroutine was generated using the symbolic computation software Maple V. Most of this output has been removed from this printed version of the UEL subroutine. The Maple worksheet that was used to produce the output that has been removed is included. The information that has been removed is reference by a lower-case letter that corresponds to the commented line on the Maple worksheet used to create the output.

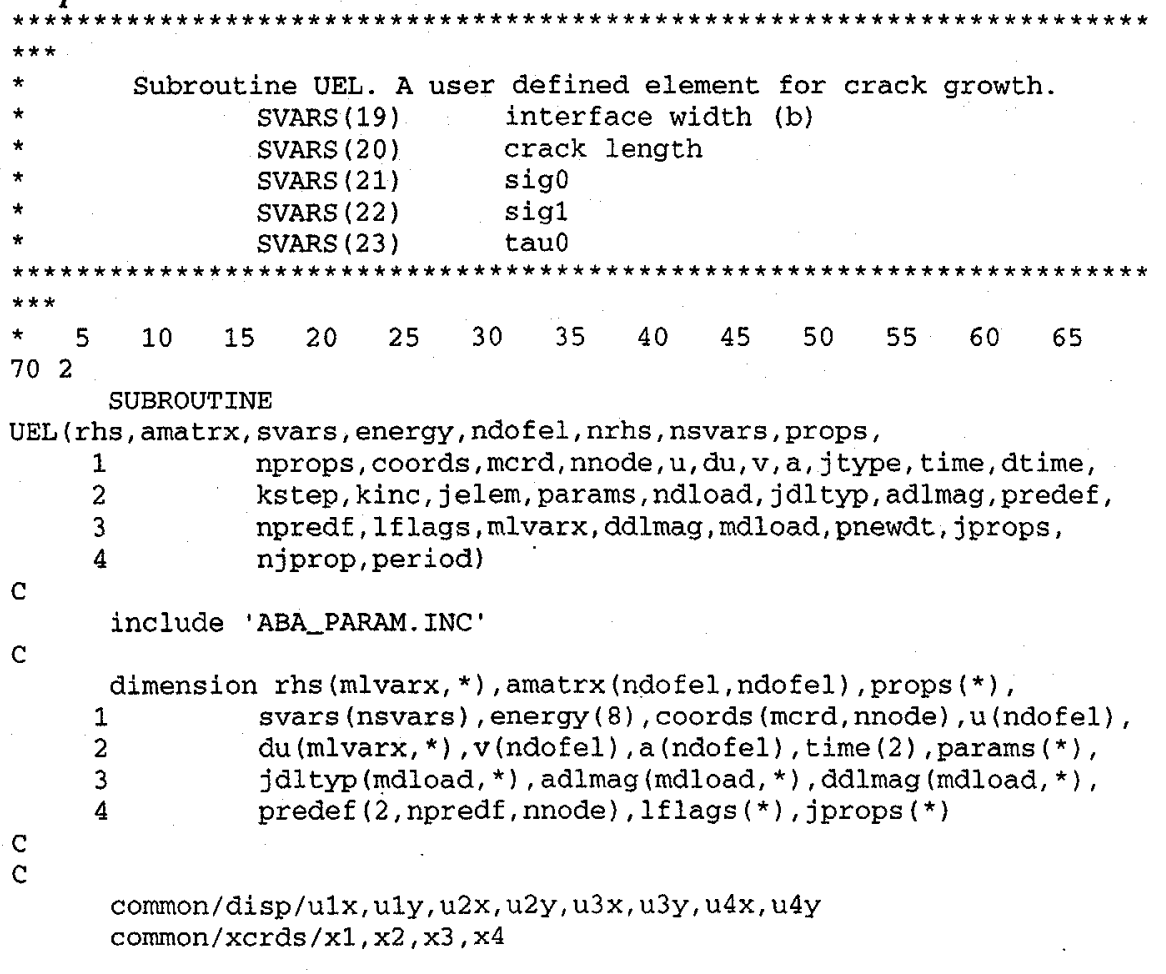

common/ycrds/y1,y2,y3,y4

common/bdy/bx, by

common/thck/thk1, thk2, thk3, thk

common/prps/em, gnu, g1c,ac, rho, sigult, iadj1, iadj2

common/dim/b, h

common/iter/iequiliter

common/plane/iplnstrn, iplnstrss

common/sigtau/sig0,sig1, tau0

common/fail/ifailf (100000), nodefail (100000),jfail, ifli

common/converge/duo $(100000,16)$, ddum, dum

dimension $r k(8,8)$, epsilon $(100000,3)$, sigma (3), a0 (100000),

\# ivfeel $(100000,2), \operatorname{thk}(100000,8), \mathrm{bf}(100000,2)$, inewel $(100000)$.

\# ftop (8), f3 (16), evfe (3), eps (3), iold(100000), rmsmt (8,8).

wel (100000), deltaatot $(100000,1000)$, istiff(100000), rksave $(16,16$

\# rmsmt2 $(16,16), g(16,100000), g 0 l d(16,100000), r k u(8)$

\# ftopold $(16,100000), \operatorname{sig}(3)$, resid $(16,1), g 2(16,100000), \operatorname{sxx}(2)$.

\# $\operatorname{sxy}(2)$, ftop2 $(16,100000)$, syy (2)

\# $\operatorname{sxy}(2)$, ftop2 $(16,100000), \operatorname{syy}(2)$
data idebug $/ 1 /$, ientry $/ 0 /, \mathrm{kincold} / 0 /, \mathrm{kequil} / 0 /$, iequiliter $/ 1 /$ $* * *$
* 5

\section{if (ientry.eq. 0 ) then}

open (unit $=81$, file $=$ "/raid5/gerken/abqus $/$ uel $/$ body")

open (unit $=80$, file $="$ raid5 $/$ gerken $/$ abqus $/$ uel $/$ thick")

open (unit=82, file=" /raid5/gerken/abqus/uel/vfedeck")

$\operatorname{read}(80, *)$ noel

do 101 ii=1, noel

read $(80, *)$ ielno, thk (ielno, 1$)$, thk (ielno, 2), thk(ielno, 3),

1 thk (ielno, 4)

101 continue

$\operatorname{read}(81, *)$ noel

do $111 j j=1$, noe

read $(81, *)$ ielno, bf (ielno, 1), bf (ielno, 2)

111 continue

$\operatorname{read}(82, *)$ numvfe

do $121 \mathrm{kk}=1$, numvfe

$\operatorname{read}(82, *)$

ivfeno, ivfeel (ivfeno, 1), ivfeel (ivfeno, 2), a0 (ivfeno)

iold (ivfeno) $=1$

continue 


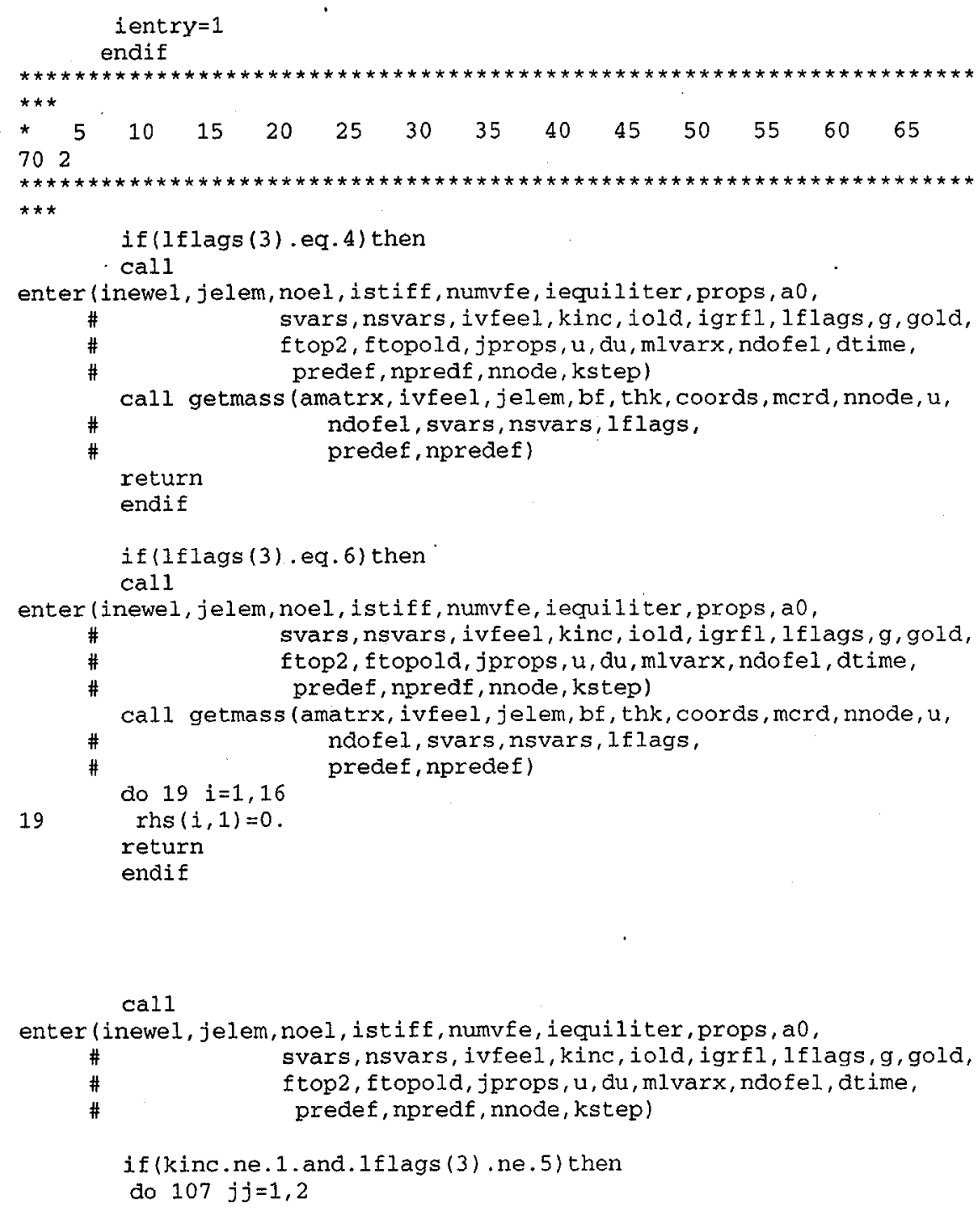

if $(k$ kinc.ne.1.and.1flags (3). ne.5) then do $107 j j=1,2$

call xyuvbh ( $j j$, coords, mcrd, nnode, $u$, ndofel, svars, nsvars) call strss (sigma)

$\mathbf{s X}=\mathbf{s i g m a}(1)$

sy=sigma $(2)$

txy=sigma (3)

call transformsig ( $u$, ndofel, coords, mcrd, nnode, sx, sy, txy)

$\operatorname{sxx}(j j)=s x$

$\operatorname{syy}(j j)=s y$

$\operatorname{sxy}(j j)=t x y$

if $(j j$. eq. 2$)$ then

sig $0=(\operatorname{syy}(1)+\operatorname{syy}(2)) / 2$

$\operatorname{sig} 1=(\operatorname{sxx}(1)+\operatorname{sxx}(2)) / 2 .-\operatorname{sig} 0$

$\operatorname{tau} 0=(\operatorname{sxy}(1)+\operatorname{sxy}(2)) / 2$

svars $(21)=\operatorname{sig} 0$

svars $(21)=\operatorname{sig} 0$

$\operatorname{svars}(22)=\operatorname{sig} 1$
$\operatorname{svars}(23)=\operatorname{tau} 0$

call aupdate (sigo, tauo, wtot, dat, del taa)

if ( (aO (jelem)+deltaa).gt. svars (20)) then

$a c=a 0(j e l e m)+$ deltaa

else

$\mathrm{ac}=\mathrm{svars}(20)$

endif

if (sig0.ge.sigult) then

$\mathrm{ac}=\mathrm{b}+10$.

endif

\section{endif \\ continue \\ endi}

* Structural element contributions

$* * *$

do $16 i=1,16$

do $15 j=1,16$

rmsmt $2(i, j)=0$

rksave $(i, j)=0$.

if (i.le.8.and.j.1e.8) then

$\operatorname{rmsmt}(i, j)=0$

$r k(i, j)=0$.$$
\text { endif }
$$

continue 
node $1=$ predef $(1,2,1) / 1$

node $5=$ predef $(1,2,5) / 1$

if (kinc.eq. 1. and.svars (20).gt.b) then

nodefail (nodel) $=1$

nodefail (node5) $=1$

ifailf $($ jelem $)=1$

endif

do 100 i $i=1,2$

call bdythk (ivfeel, jelem, ii,bf, thk)

call xyuvbh (ii, coords, merd, nnode, u, ndofel, svars, nsvars)

if (Iflags (1) .eq.11.or.lflags (1) .eq.12) then

call mm (rmsmt, rmass)

if(istiff (ivfeel (jelem, ii)) .eq.0) then

$$
\text { do } 109 i=1,8
$$$$
\text { do } 109 j=1,8
$$

$\operatorname{rmsmt} 2(i+8 *(i i-1), j+8 *(i i-1))=\operatorname{rmsmt}(i, j)$

$$
\text { endif }
$$$$
\text { endif }
$$

cal1 hwe (kinc, eps, sig, rk, f3)

e1=eps (1)

e $=$ eps (2)

e $2=\cos (3)$

$\operatorname{si=} \operatorname{sig}(1)$

s $2=\operatorname{sig}(2)$

s12=sig( 3 )

call transformeb (u, ndofel, coords, mcrd, nnode, e1, e2, e12)

eps $(1)=e 1$

eps $(2)=e 2$

eps $(3)=e 12$

li transformeb (u, ndofel, coords, mcrd, nnode, s1, s2, s12)

$\operatorname{sig}(1)=s 1$

$\operatorname{sig}(2)=s 2$

$\operatorname{sig}(3)=s 12$

do $106 j j=1,3$

106

epsilon (ivfeel (jelem, ii), jj) =eps $(j j)$

continue

C AMATRX

$c$ alpha $=$ params $(1)$

alpha $=$ params $(1)$

if (lflags (1) eq. 1 . or. Iflags.(1) eq . 2 ) beta $=1$.

if (istiff (ivfeel (jelem, ii)).eq.0) then

dt $=$ dt ime

if ( 1 flags (3) .eq. 5$) d t=d t i m e / 2$.

do $11 i=1,8$

do $10 j=1,8$

amatrx $(i+8 *(i i-1), j+8 *(i-1))=(1,+a l p h a) * r k(i, j)+$ $($ rmsmt $2(i+8 *(i i-1), j+8 *(i i-1))) *(2 . / d t * * 2$.

rksave $(i+8 *(i i-1), j+8 *(i i-1))=r k(i, j)$

continue

continue

istiff (ivfeel (jelem, ii) $)=1$

endif

do $13 i=1,8$

sum $=0$.

do $14 j=1,8$

sum $=$ sum+rksave $(i+8 *(i i-1), j+8 *(i i-1)) * u(j+8 *(i j-1)$

14 continue

$g(i+8 *(i i-1), j e l e m)=$ sum

13 continue

C RHS

C

do $35 i=1,8$

sum $=0$.

do $30 \quad j=1,8$

sum $=$ sum+rmsmt $2(i+8 *(i i-1), j+8 *(i j-1)) * a(j+8 *(i i-1))$

30 continue

rhs $(i+8 *(i i-1), 1)=-($ sum $)-(1,+$ alpha $) * g(i+8 *(i i-1), j e l e m)$

$\#$

35 continue alpha*gold $(i+8 *(i i-1)$, jelem

100 continue

$* * * * * *$

* Interface Failure

*

$\star \star \star$ 


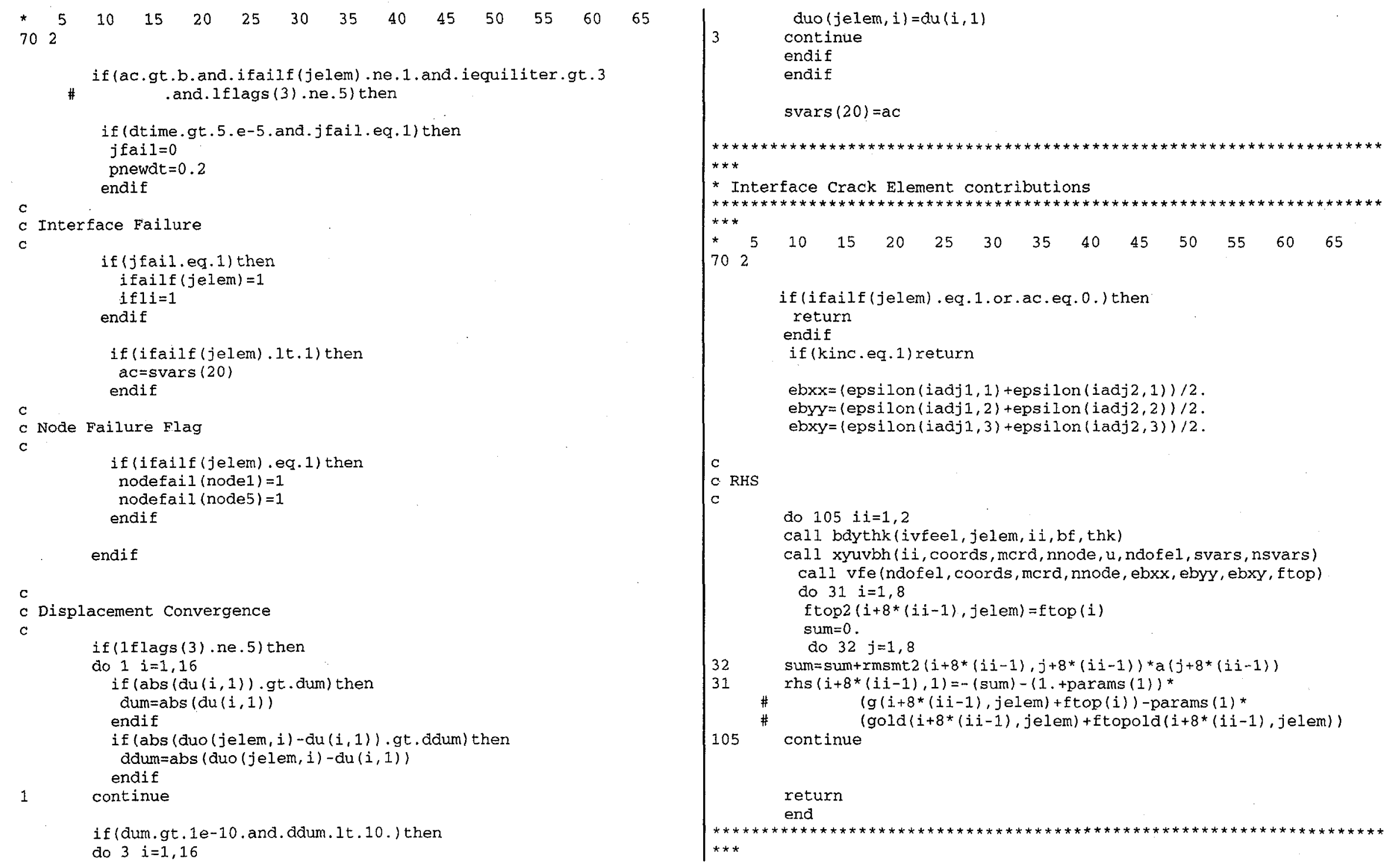


* Subroutine HWE

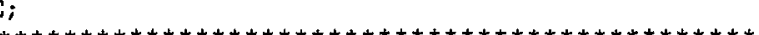

* 5

702 subroutine hwe (inc, eps, sigma, $r k, f 3)$

include 'ABA PARAM. INC'

dimension sigma (3)

call stiff(rk)

call load(f3)

call strn (eps)

call strss(sigma)

return

end

$\star * * * * * *$

$\star \star \star *$

* Subroutine vfe

* Calculates and returns the load vectors associated

* with the elements adjacent to the vfe.

$* * *$

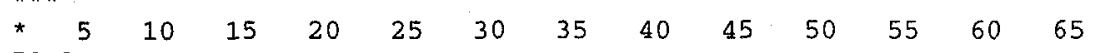

702

subroutine vfe (ndofel, coords, merd, nnode, ebxx, ebyy, ebxy, gahaf2) include 'ABA_PARAM. INC'

common $/ x \operatorname{crds} / x 1, x 2, x 3, x 4$

common/ycrds $/ y^{1}, y^{2}, y^{3}, y^{4}$

common/thck/thk1, thk2, thk 3 , thk 4

common/prps/em, gnu, glc,ac, rho, sigult, iadj1, iadj2

common/dim/b, h

common/sigtau/sig0,sig1, tau0

dimension $f(8), f 2(9), f 2 i(9), g w(10), g p(10), t x(4), t y(4)$

dimension gahaf $2(8)$, gaha $(8,9)$, evfe $(3), \operatorname{ev}(3)$, st $(9,3)$

gp $(1)=0.861136311594953$

$\mathrm{gp}(2)=0.339981043584856$

$\mathrm{gp}(3)=-0.861136311594953$

$g p(4)=-0.339981043584856$

gw $(1)=0.347854845137454$

gW (2) $=0.652145154862546$

gW (3) $=0.347854845137454$

gw $(4)=0.652145154862546$ do $70 j j=1,3$

$\operatorname{ev}(j j)=0$.

continue

Transform to Local Interface Coordinates

call transformxy ( $u$, ndofel, coords, merd, nnode, tx, ty)

c Gauss Quadrature to integrate evfe( ()${ }^{\star} \operatorname{det}(\mathrm{J})$ *thick

$$
\begin{aligned}
& \text { do } 10 i=1,4 \\
& \text { do } 20 j=1,4 \\
& \text { if }(g p(i) .1 t .0 .) \text { then } \\
& \text { xisign=-1. } \\
& \text { else } \\
& \text { xisign=1. } \\
& \text { endif } \\
& \text { xi=gp (i) } \\
& \text { eta }=(g p(j))+1 . \\
& \text { call dj(xi, eta, det } j)
\end{aligned}
$$

thick $=\operatorname{thk} 1 *(1-x i) *(1 .-$ eta $) / 4 .+$ thk $2 *(1 .+x i) *(1 .-$ eta $) / 4 .+$ $\# \operatorname{thk} 3 *(1,+x i) *(1,+e t a) / 4,+\operatorname{thk} 4 *(1,-x i) *(1,+e t a) / 4$

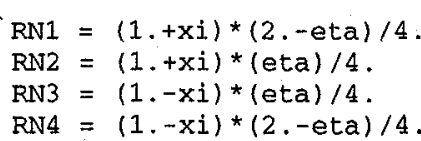

$\mathrm{RN1}=(1,+x i) *(2 .-$ eta $) / 4$

RN2 $=(1 .+x i) *(e t a) / 4$

$\mathrm{RN} 3=(1 .-\mathrm{xi}) *($ eta $) / 4$

RN4 $=(1,-x i) *(2,-$ eta $) / 4$

$x=a b s(R N 1 * t x(1)+R N 2 * \operatorname{tx}(2)+R N 3 * t x(3)+R N 4 * t x(4))$

$y=R N 1 * t y(1)+R N 2 * t y(2)+R N 3 * t y(3)+R N 4 * t y(4)$

call sevfe (xisign, $x, y$, ebxx, ebyy, ebxy, evfe)

c Transform Strain to global coordinates

cal1 transformevfe (u, ndofel, coords, mcrd, nnode, evfe)

do $30 \quad i j=1,3$

ev $(i j)=e v(i j)+g w(i){ }^{*} g w(j) * \operatorname{evfe}(i j) * t h i c k$ det $j$

continue 
call stj(st)

do $31 i=1,9$

$$
\text { sum }=0 \text {. }
$$

do $21 j=1,3$

$\operatorname{sum}=\operatorname{sum}+s t(i, j) * \operatorname{ev}(j)$

21

continue

$f 2 i(i)=$ sum

31 continue

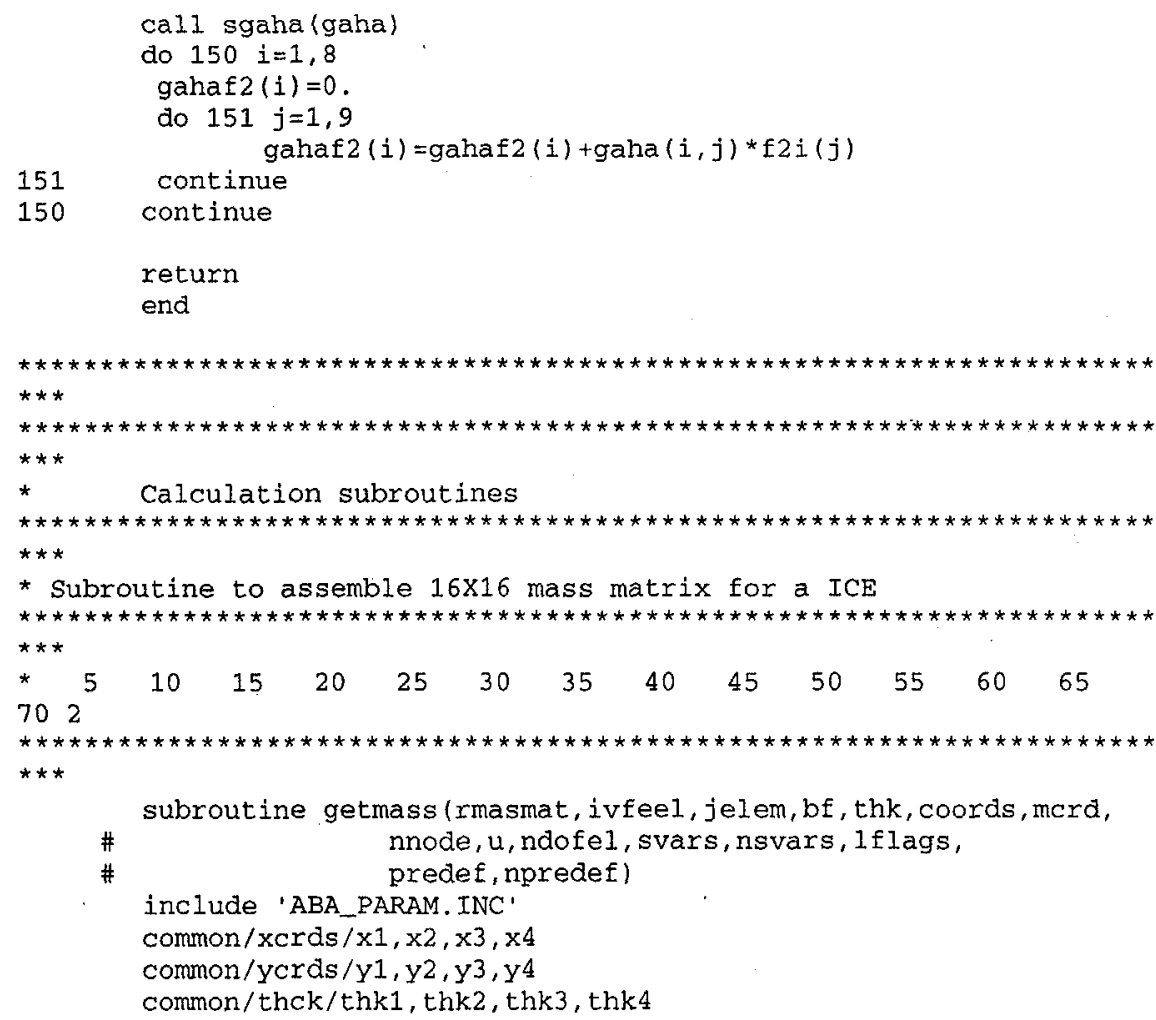

common/prps/em, gnu, g1c, ac, rho, sigult, iadj1, iadj2

dimension rmasmat $(16,16), \operatorname{rmsmt}(8,8), \operatorname{massflag} 4(50000)$

\# ivfeel $(100000,2)$, massflag6 (50000), lflags (*).

\# predef $(2$, npredef, nnode)

if (1flags (3) .eq. 4 ) then

do $10 i i=1,2$

call xyuvbh (ii, coords, mcrd, nnode, $u$, ndofel, svars, nsvars call bdythk (ivfeel, jelem, ii, bf, thk)

call mm (rmsmt, rmass)

if (massflag4 (ivfeel (jelem, ii)) .ne.1) then

do $20 j j=1,8$.

do $20 \mathrm{kk}=1,8$

$20 \quad \operatorname{rmasmat}(j j+8 *(i i-1), k k+8 *(i i-1))=\operatorname{rmsmt}(j j, k k)$ Imasm

massflag 4 (ivfeel (jelem, ii) $)=1$

continue

elseif(1flags ( 3 ).eq. 6) then

do $30 \mathrm{i} i=1,2$

call xyuvbh ( $i \mathrm{i}$, coords, mcrd, nnode, $u$, ndofel, svars, nsvars)

call bdythk (ivfeel, jelem, ii, bf, thk)

if (massflag6 (ivfeel (jelem, ii)) ne.1) then

call $\mathrm{mm}$ (rmsmt, rmass)

do $40 j j=1,8$

do $40 \mathrm{kk}=1,8$

$\operatorname{rmasmat}(j j+8 *(i i-1), k k+8 *(i j-1))=\operatorname{rmsmt}(j j, k k)$

endif

massflag $6($ ivfeel $(j e l e m, i i))=1$

continue

endif

return

end

$* * *$

* Subroutine to assign body forces and thicknesses

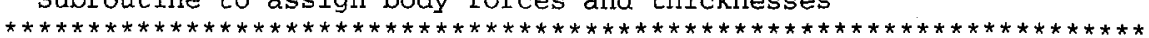

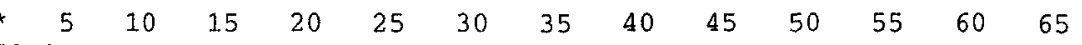

702 subroutine bdythk (ivfeel, jelem, $i$, bf, thk) 
include 'ABA_PARAM. INC'

dimension thk $(100000,8)$, bf $(100000,2)$, ivfeel $(100000,2)$ common/bdy/bx, by

common/thek/thk1, thk 2 , thk 3 , thk 4

$\mathrm{bx}=\mathrm{bf}(\mathrm{ivfeel}(\mathrm{jelem}, \mathrm{ii}), 1)$

by=bf (ivfeel (jelem, il), 2 )

thk1=thk (ivfeel (jelem, ii), 1)

thk $2=$ thk (ivfeel (jelem, ii), 2 )

thk $3=$ thk (ivfeel (jelem, ii), 3)

thk $4=$ thk (ivfeel (jelem, $i$ i) , 4 )

\section{return}

end

$* * *$

* Subroutine to assign coordinates and displacements

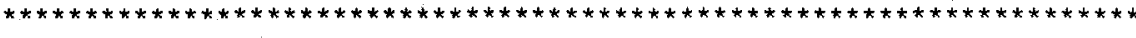

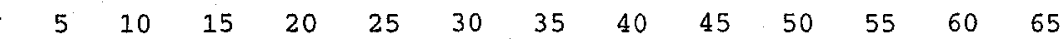

702

subroutine xyuvbh(i, coords, mcrd, nnode, $u$, ndofel, svars, nsvars) include 'ABA_PARAM. INC

dimension coords (mcrd, nnode), $u$ (ndofel), svars (nsvars)

common/disp/u1x, u1y,u2x,u2y,u3x,u3y,u4x,u4y

common $/ \mathrm{xcrds} / \mathrm{x} 1, \mathrm{x} 2, \mathrm{x} 3, \mathrm{x} 4$

common $/ y \operatorname{crds} / \mathrm{y} 1, \mathrm{y} 2, \mathrm{y}^{3}, \mathrm{y} 4$

common $/ \mathrm{dim} / \mathrm{b}, \mathrm{h}$

$u 1 x=u(1+8 *(i-1))$

$\mathrm{uly}=\mathrm{u}(2+8 *(i-1))$

$\mathrm{u} 2 \mathrm{x}=\mathrm{u}(3+8 *(\mathbf{i}-1))$

$\mathrm{u} 2 \mathrm{y}=\mathrm{u}\left(4+8^{*}(i-1)\right)$

$\mathrm{u} 3 \mathrm{x}=\mathrm{u}(5+8 *(\mathrm{i}-1))$

$u 3 y=u(6+8 *(i-1))$

$u 4 x=u(7+8 *(i-1))$

$\mathrm{u} 4 \mathrm{y}=\mathrm{u}(8+8 *(i-1))$

$x 1=\operatorname{coords}(1,1+4 *(i-1))$

$\mathrm{x} 2=\operatorname{coords}(1,2+4 *(i-1))$

$\times 3=\operatorname{coords}(1,3+4 *(i-1))$

$\mathrm{x} 4=\operatorname{coords}(1,4+4 *(i-1)$

recoord

$y 1=\operatorname{coords}(2,1+4 *(i-1))$

$y^{2}=\operatorname{coords}\left(2,2+4^{*}(i-1)\right)$

$\mathrm{y} 3=\operatorname{coords}(2,3+4 *(i-1))$

$y 4=\operatorname{coords}(2,4+4 *(i-1))$

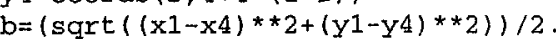

$h=(\operatorname{sqrt}((x 1-x 2) * \star 2+(y 1-y 2) * * 2))$

svars (19) $=b$

end

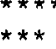

* Subroutine called upon entry into uel subroutine

$*$

702

$\#$

subroutine enter(inewel, jelem, noel, istiff, numvfe, iequiliter,

props, a0, svars, nsvars, ivfeel, kinc, iold, igrf 1

lflags, g, gold, ftop2, ftopold, jprops, u, du, m1varx

ndofel, dtime, predef, npredf, nnode, kstep)

include 'ABA PARAM. INC

dimension inewel $(100000)$, istiff $(100000)$, props $(*), a 0(100000)$ \#. svars(nsvars), ivfeel $(100000,2)$, iold $(100000)$, lflags (*). \# $\operatorname{gold}(16,100000), g(16,100000)$, ftop2 $(16,100000)$,

\# ftopold $(16,100000), j$ props (*), u (ndofel), du (mlvarx, *),

\# $\quad$ predef ( 2 , npredf, nnode)

common/prps/em, gnu, g1c, ac, rho, sigult, iadj1, iadj2

common/fail/ifailf (100000), nodefail(100000), jail, ifli

common/plane/iplnstrn, iplnstrss

common/converge/duo $(100000,16)$, ddum, dum

c Element Properties

iadj $1=i v f e e l(j e l e m, 1)$

iadj2=ivfeel (jelem, 2 )

em=props (1)

$\mathrm{gnu}=$ props $(2)$

gic=props $(3)$

rho=props (4)

sigult=props (5)

if (jprops (1) .eq.1) then

iplnstrn $=0$

iplnstrss $=1$

elseif (jprops (1) .eq.2) then

iplnstrn=1

iplnstrss $=0$

endif 
C Initial Failure

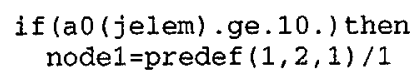

nodefail $($ node 1$)=1$

nodefail (node5) $=1$

\section{ifailf $($ jelem $)=1$}

endif

if (1flags ( 3 ).eg. 4 ) return

\section{if (kinc. eq. 1) then}

ac $=a 0$ (jelem)

$\operatorname{svars}(20)=a 0(j e l e m)$

else

ac=svars $(20)$

endif

c New Iteration

if (inewel (jelem) .eq.1) then

$$
\text { do } 200 i=1 \text {, noe } 1
$$$$
\text { istiff }(i)=0
$$

continue

do $201 j=1$, numvere

inewel $(j)=0$

201

continue

c Solution Convergence

if (lflags ( 3 ) . ne. 5 ) then

if (dum.gt.0.and. ddum.gt.0.) then

if (ddum/dum.1t.1e-2) then.

$j$ fail $=1$

else

$j$ fail $=0$

endif if (ddum/ddumo.1t. $1 \mathrm{e}-9$ ) j fail $=0$

if (ddum/ddumo.gt. 1 . ) jfail $=0$

if (dum. It. 1e-15) jfail $=0$

if (dum.gt.1.e-1) jfail=0

if (ddum. It . 1e-15) j fai $1=0$

if (daum.gt. 1.) jfail $=0$

if (iequiliter. le 4 ) jfail $=0$

if (ifli.eq.1) jfail=0

else

jfail $=0$

endif

adumo=đaum

if (ddumo . eq . 0.) ddumo $=1$.

dumo $=$ dum

ddum $=0$.

dum $=0$.

iequiliter $=$ iequiliter +1

$i c=i c+1$

if (ic.ge.2) then

if $1 i=0$

ic $=0$

endif

endif

endif

inewel $(j e l e m)=1$

c New Increment
if ( (kinc-1). eq.iold (jelem)) then
do $203 i=1$, numvfe

iold $(i)=k i n c$

do $205 i=1$, numvfe

do $204 j=1,16$

ftopold $(j, i)=f \operatorname{top} 2(j, i)$

$\operatorname{gold}(j, i)=g(j, i)$

continue

205 continue

iequiliter $=1$

endif 


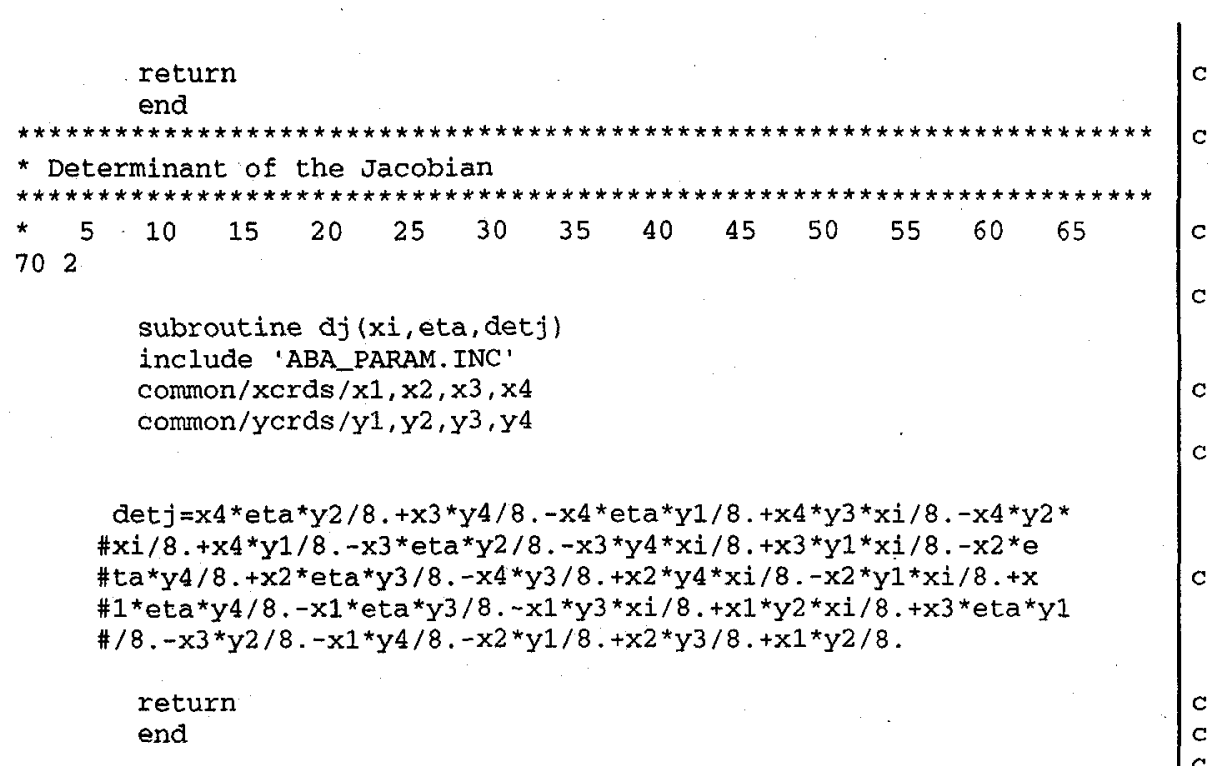

* Transformation Matrix

* Note: Set up for 3-D but only used for 2-D

* 5 - 50 702

subroutine sq(u, ndofel, coords, mcra, nnode, $q)$

include 'ABA_PARAM. INC'

common $/ x \operatorname{crds} / x 1, x 2, x 3, x 4$

common/yerds/y1, $y_{2}, y^{3}, y 4$

dimension $q(2,2)$, coords (mcrd, nnode), u (ndofel)

$c$ out of plane vectors on neighboring shell elements

c connecting shell

plx $=\operatorname{coords}(1,1)+u(1)$

$\mathrm{p} 1 \mathrm{y}=\operatorname{coords}(2,1)+\mathrm{u}(2)$

$\mathrm{p} 1 \mathrm{z}=\operatorname{coords}(3,1)$

$\mathrm{plz}=0$.

$\mathrm{p} 2 \mathrm{x}=\operatorname{coords}(1,3)+\mathrm{u}(5)$
$2 y=\operatorname{coords}(2,3)+12(6)$

$p^{2} z=\operatorname{coords}(3,3)$

$\mathrm{p} 2 z=0$.

$\mathrm{p} 3 \mathrm{x}=\operatorname{coords}(1,2)+\mathrm{u}(3)$ p $3 y=\operatorname{coords}(2,2)+u(4)$

p3z $=\operatorname{coords}(3,2)$

$\mathrm{p} 3 \mathrm{z}=0$.

$\mathrm{p} 4 \mathrm{x}=\operatorname{coords}(1,4)+\mathrm{u}(7)$

$\mathrm{p} 4 \mathrm{y}=\operatorname{coords}(2,4)+\mathrm{u}(8)$

$\mathrm{p} 4 \mathrm{z}=\operatorname{coords}(3,4)$

$\mathrm{p} 4 \mathrm{z}=0$.

$\mathrm{v} 1 \mathrm{x}=\mathrm{p} 2 \mathrm{x}-\mathrm{p} 1 \mathrm{x}$

$v 1 y=p 2 y-p 1 y$

$\mathrm{v} 1 \mathrm{z}=\mathrm{p} 2 \mathrm{z}-\mathrm{p} 1 \mathrm{z}$

$\mathrm{v} 2 \mathrm{x}=\mathrm{p} 4 \mathrm{x}-\mathrm{p} 3 \mathrm{x}$

$\mathrm{v} 2 \mathrm{y}=\mathrm{p} 4 \mathrm{y}-\mathrm{p} 3 \mathrm{y}$

$\mathrm{v} 2 \mathrm{z}=\mathrm{p} 4 \mathrm{z}-\mathrm{p} 3 \mathrm{z}$

cross $\mathrm{v} 1$ and $\mathrm{v} 2$

$\mathrm{vn} 1 \mathrm{x}=\mathrm{v} 1 \mathrm{y} * \mathrm{v} 2 \mathrm{z}-\mathrm{v} 1 \mathrm{z}^{\star} \mathrm{v} 2 \mathrm{y}$

$\mathrm{vn} 1 \mathrm{y}=\mathrm{v} 1 \mathrm{z} * \mathrm{v} 2 \mathrm{x}-\mathrm{v} 1 \mathrm{x} * \mathrm{v} 2 \mathrm{z}$

$\mathrm{vn} 1 \mathrm{z}=\mathrm{v} 1 \mathrm{x}^{*} \mathrm{v} 2 \mathrm{y}-\mathrm{v} 1 \mathrm{y}^{*} \mathrm{v} 2 \mathrm{x}$

connecting shell

$\mathrm{p} 1 \mathrm{x}=\operatorname{coords}(1,5)+u(9)$

ply $=\operatorname{coords}(2,5)+u(10)$

$\mathrm{p} 1 \mathrm{z}=\operatorname{cooras}(3,5)$

$\mathrm{p} 1 \mathrm{z}=0$.

$\mathrm{p} 2 \mathrm{x}=\operatorname{coords}(1,7)+\mathrm{u}(13)$

p2y $=$ coords $(2,7)+\mathrm{u}(14)$

$p 2 z=\operatorname{coords}(3,7)$

$\mathrm{p} 2 \mathrm{z}=0$.

$p 3 x=\operatorname{coords}(1,6)+u(11)$

p3y $=\operatorname{coords}(2,6)+u(12)$

$\mathrm{p} 3 \mathrm{z}=\operatorname{coords}(3,6)$

$\mathrm{p} 3 z=0$.

$\mathrm{p} 4 \mathrm{x}=\operatorname{coords}(1,8)+\mathrm{u}(15)$ 
$\mathrm{p} 4 \mathrm{y}=\operatorname{coords}(2,8)+\mathrm{u}(16)$

$\mathrm{p} 4 \mathrm{z}=\operatorname{coords}(3,8)$

$\mathrm{p} 4 \mathrm{z}=0$

$\mathrm{v} 1 \mathrm{x}=\mathrm{p} 2 \mathrm{x}-\mathrm{p} 1 \mathrm{x}$

$v 1 y=p 2 y-p 1 y$

$\mathrm{v} 1 z=\mathrm{p} 2 z-\mathrm{p} 1 \mathrm{z}$

c

$\mathrm{v} 2 \mathrm{x}=\mathrm{p} 4 \mathrm{x}-\mathrm{p} 3 \mathrm{x}$

$\mathrm{v} 2 \mathrm{y}=\mathrm{p} 4 \mathrm{y}-\mathrm{p} 3 \mathrm{y}$

$\mathrm{v} 2 \mathrm{z}=\mathrm{p} 4 \mathrm{z}-\mathrm{p} 3 \mathrm{z}$

$c$ cross $v 1$ and $v 2$

$\mathrm{vn} 2 \mathrm{x}=\mathrm{v} 1 \mathrm{y}^{*} \mathrm{v} 2 \mathrm{z}-\mathrm{v} 1 \mathrm{z}^{*} \mathrm{v} 2 \mathrm{y}$

$\mathrm{vn} 2 \mathrm{y}=\mathrm{v} 1 \mathrm{z}^{\star} \mathrm{v} 2 \mathrm{x}-\mathrm{v} 1 \mathrm{x}^{\star} \mathrm{v} 2 \mathrm{z}$

$\mathrm{vn} 2 z=v 1 x^{\star} v 2 y-v 1 y^{\star} v 2 x$

$\mathrm{C}$ add vectors unit vectors $\mathrm{vn} 1$ and $\mathrm{vn} 2$

$$
\begin{aligned}
& \text { vtx }=\operatorname{vn} 1 x+\operatorname{vn} 2 x \\
& \text { vty }=\operatorname{vn} 1 y+v n 2 y
\end{aligned}
$$$$
\begin{aligned}
& \text { vty }=\text { vnly }+ \text { vn } 2 y \\
& \text { vtz }=\operatorname{vn} 1 z+\operatorname{vn} 2 z
\end{aligned}
$$

$\operatorname{vmag}=\operatorname{sqrt}\left(v t x x^{\star} 2+v t y * \star 2+v t z * \star 2\right)$

if (vmag. le.0) vmag $=1$.

$c$ thus, the out of plane vector, local $\mathrm{z}$ direction

$\mathrm{rlx}=\mathrm{vtx} / \mathrm{vmag}$

$\mathrm{r} 1 \mathrm{y}=\mathrm{vty} / \mathrm{vmag}$

$\mathrm{rlz}=\mathrm{vtz} / \mathrm{vmag}$

c let local $x$ direction be vector $(i-j)$ of the VFE

$\mathrm{p} 1 \mathrm{x}=\operatorname{coords}(1,4)$

$\mathrm{ply}=\operatorname{coords}(2,4)$

$\mathrm{p} 1 \mathrm{z}=\operatorname{coords}(3,4)$

$\mathrm{p} 1 \mathrm{z}=0$

$\mathrm{C}$

$\mathrm{p} 2 \mathrm{x}=\operatorname{coords}(1,1)$

p2y $=\operatorname{coords}(2,1)$

$\mathrm{p} 2 \mathrm{z}=\operatorname{coords}(3,1)$

$\mathrm{p} 2 \mathrm{z}=0$ $\mathrm{v} 1 \mathrm{y}=\mathrm{p} 2 \mathrm{y}-\mathrm{p} 1 \mathrm{y}$

$\mathrm{v} 1 \mathrm{z}=\mathrm{p} 2 \mathrm{z}-\mathrm{p} 1 \mathrm{z}$

vmag $=\operatorname{sqrt}\left(v 1 x^{* * 2}+\mathrm{v} 1 y^{\star *} 2+\mathrm{v} 1 \mathrm{z}^{\star \star} 2\right)$

if (vmag.le.0) vmag $=1$.

$\mathrm{r} 2 \mathrm{x}=\mathrm{v} 1 \mathrm{x} / \mathrm{vmag}$

$\mathrm{r} 2 \mathrm{y}=\mathrm{v} 1 \mathrm{y} / \mathrm{vmag}$

$\mathrm{r} 2 \mathrm{z}=\mathrm{v} 1 \mathrm{z} / \mathrm{vmag}$

$c$

c cross $r 1$ and $r 2$

$r 3 \mathrm{x}=r 1 \mathrm{y}^{*} r 2 \mathrm{z}-r 1 \mathrm{z}^{*} r 2 \mathrm{y}$

$x 3 \mathrm{y}=\mathrm{r} 1 \mathrm{z}^{\star} \mathrm{r} 2 \mathrm{x}-\mathrm{r} 1 \mathrm{x}^{\star} \mathrm{r} 2 \mathrm{z}$

$r 3 z=r 1 x^{\star} r 2 y-r 1 y^{\star} r 2 x$

c

$\mathrm{c} 1 \mathrm{x}=\mathrm{r} 2 \mathrm{x}$

$\mathrm{c} 1 \mathrm{y}=\mathrm{r} 2 \mathrm{y}$

$\mathrm{c} 1 \mathrm{z}=\mathrm{r2z}$

c.

$\mathrm{c} 2 \mathrm{y}=\mathrm{r} 3 \mathrm{y}$

$\mathrm{c} 2 \mathrm{z}=\mathrm{r} 3 \mathrm{z}$

$\mathrm{c} 3 \mathrm{x}=\mathrm{r} 1 \mathrm{x}$

$\mathrm{c} 3 \mathrm{y}=\mathrm{r} 1 \mathrm{y}$

$\mathrm{c} 3 \mathrm{z}=\mathrm{r} 1 \mathrm{z}$

c establish the transformation matrix

base system XYZ to local system xyz$$
\begin{aligned}
& q(1,1)=c 1 x \\
& q(1,2)=c 1 y
\end{aligned}
$$

$\mathrm{q}(1,3)=\mathrm{c} 1 \mathrm{z}$

$q(2,1)=c 2 x$

$q(2,2)=c 2 y$

$q(2,3)=c 2 z$

$q(3,1)=c 3 x$

$q(3,2)=c 3 y$

$q(3,3)=c 3 z$

determinant of the transformation matrix

c $\quad x 1=q(1,1) *(q(2,2) * q(3,3)-q(2,3) * q(3,2))$

$x 2=q(1,2) *(q(2,1) * q(3,3)-q(2,3) * q(3,1))$ 
$x 3=q(1,3) *(q(2,1) * q(3,2)-q(2,2) * q(3,1))$ $\operatorname{det}=x_{1}-x_{2}+x^{3}$

return

end

* Transform Coordinates

*********************************************************************)

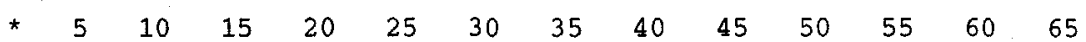

702

subroutine transformxy $(u$, ndofel, coords, mcrd, nnode, $x, y)$

include 'ABA_PARAM. INC'

common $/ \mathrm{xcrds} / \mathrm{x} 1, \mathrm{x} 2, \mathrm{x} 3, \mathrm{x} 4$

common/ycrds/y1, $\mathrm{y}_{2}, \mathrm{y} 3, \mathrm{y} 4$

dimension $q(2,2)$, coords (mord, nnode), $u$ (ndofel), $x(4), y(4)$

call sq (u, ndofel, coords, mcrd, nnode, $q$ )

$x \times 1=x 1-(x 1+x 4) / 2$.

$x \times 2=x 2-(x 1+x 4) / 2$

$x \times 3=x 3-(x 1+x 4) / 2$

$\mathrm{x} x 4=\mathrm{x} 4-(\mathrm{x} 1+\mathrm{x} 4) / 2$

$\mathrm{yy} 1=\mathrm{y}^{1}-(\mathrm{y} 1+\mathrm{y} 4) / 2$

$y y^{2}=y^{2}-(y 1+y 4) / 2$.

$\mathrm{yy}^{3}=\mathrm{y}^{3}-\left(\mathrm{y} 1+\mathrm{y}^{4}\right) / 2$

$y^{4}=y^{4}-\left(y^{1}+y^{4}\right) / 2$

$x(1)=(q(1,1) * x \times 1+q(1,2) * y y 1)$

$y(1)=(q(2,1) * x \times 1+q(2,2) * y y 1)$

$x(2)=(q(1,1) * x x 2+q(1,2) * y y 2)$

$y(2)=\left(q(2,1) * x \times 2+q(2,2) * y y^{2}\right)$

$x(3)=(q(1,1) * x \times 3+q(1,2) * y y 3)$

$y(3)=(q(2,1) * x \times 3+q(2,2) * y y 3)$

$x(4)=(q(1,1) * x x 4+q(1,2) * y y 4)$

$y(4)=(q(2,1) * x \times 4+q(2,2) * y y 4)$

return

end

* Transform Strain

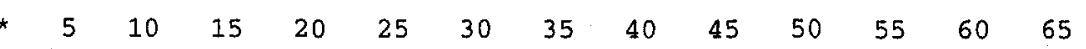

subroutine

trans formeb ( $u$, ndo fel, coords, mcrd, nnode, ebxx, ebyy, ebxy) include 'ABA_PARAM. INC'

dimension $\mathrm{eb}(2,2)$, qe $(2,2)$, qeq $(2,2), \mathrm{q}(2,2)$, coords (mard, nnode),

\# u(ndofel)

call $s q$ ( $u$, ndofel, coords, mcrd, nnode, q)

$\mathrm{eb}(1,1)=\mathrm{ebxx}$

$\mathrm{eb}(1,2)=.5^{*} \mathrm{ebxy}$

$\mathrm{eb}(2,1)=.5 * \mathrm{ebxy}$

$\mathrm{eb}(2,2)=\mathrm{ebyy}$

do $10 \quad i=1,2$

do $20 j=1,2$

$$
\text { qe }(i, j)=0 \text {. }
$$

$q e q(i, j)=0$

20 continue

continue

do $30 \quad k=1,2$

do $40 \quad 1=1,2$

do $50 \mathrm{~m}=1,2$

$q e(k, 1)=q e(k, 1)+q(k, m) * e b(m, 1)$

50 continue

$$
\text { continue }
$$

continue

do $60 \quad i j=1,2$

do $70 j j=1,2$

geq $(i i, j j)=q e q(i i, j j)+q e(i i, k k) * q(j j, k k)$

80 continue

continue

continue

ebxx=qeq $(1,1)$

ebyy $=q e q(2,2)$

ebxy $=2 .{ }^{*}$ geg $(2,1)$

return

end

rend

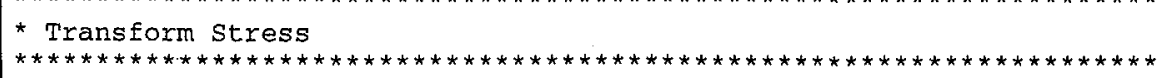


subroutine transformsig ( $u$, ndofel, coords,merd, nnode, sxx, syy, sxy) include 'ABA_PARAM. INC'

dimension eb $(2,2)$, ge $(2,2)$, geg $(2,2), q(2,2)$, coords (mcrd, nnode),

\# $\mathrm{u}$ (ndofel)

call sq(u, ndofel, coords, mcrd, nnode, $q)$

$\mathrm{eb}(1,1)=\mathrm{sxx}$

$\mathrm{eb}(1,2)=s x y$

$e b(2,1)=s x y$

$\mathrm{eb}(2,2)=s y y$

do $10 \quad i=1,2$

do $20 j=1,2$

$\operatorname{qe}(i, j)=0$.

20 continue

10 continue

do $30 \mathrm{k}=1,2$

do $40 \quad 1=1,2$

$$
\text { do } 50 \mathrm{~m}=1,2
$$$$
q e(k, 1)=q e(k, 1)+q(k, m) * e b(m, 1)
$$

50 continue

40 continue

$$
\text { continue }
$$

do $60 \quad i i=1,2$

do $70 j j=1,2$

$$
\text { do } 80 \mathrm{kk}=1,2
$$$$
q \operatorname{qeq}(i i, j j)=q e q(i i, j j)+q e(i i, k k) * q(j j, k k)
$$

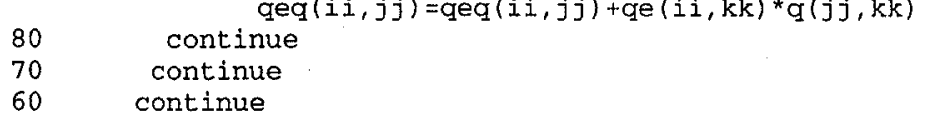

\section{$\mathrm{sxx}=$ geq $(1,1)$}

syy $=$ geq $(2,2)$

sxy=qeq $(2,1)$

\section{return}

end

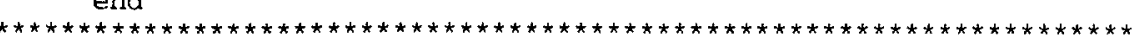

* Transform Crack Strain

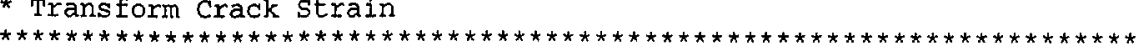

$\begin{array}{lllllllllllll}* & 5 & 10 & 15 & 20 & 25 & 30 & 35 & 40 & 45 & 50 & 55 & 60\end{array}$

702

subroutine transformevfe( $u$, ndofel, coords, mcrd, nnode, evfe) include 'ABA_PARAM. INC'

dimension $\mathrm{eb}(2,2)$, ge $(2,2)$, geq $(2,2), \mathrm{q}(2,2)$, qt $(2,2)$, evfe $(3)$ \# coords (merd, nnode), u (ndofel)

call sq( $u$, ndofel, coords, merd, nnode, qt)

$\mathrm{eb}(1,1)=\mathrm{evfe}(1)$

$\mathrm{eb}(1,2)=.5^{\star}$ evfe $(3)$

$\mathrm{eb}(2,1)=.5^{*}$ evfe( $(3)$

$\mathrm{eb}(2,2)=\operatorname{evfe}(2)$

$q(1,1)=q t(1,1)$

$q(1,2)=q t(2,1)$

$q(2,1)=q t(1,2)$

$q(2,2)=q t(2,2)$

do $20 j=1$,

ge $(i, j)=0$.

20 continue

10 continue

do $30 k=1,2$

do $40 \quad 1=1,2$

do $50 \mathrm{~m}=1,2$

continue

$q \in(k, 1)=q e(k, 1)+q(k, m) * e b(m, 1)$

continue

continue

do $60 i i=1,2$

do $70 \quad j j=1,2$

do $80 \mathrm{kk}=1,2$

$q e q(i i, j j)=q e q(i i, j j)+q e(i i, k k) * q(j j, k k)$

continue

continue

continue

$\operatorname{evfe}(1)=\operatorname{geq}(1,1)$

evfe $(2)=$ geq $(2,2)$

evfe $(3)=2$. * geg $(1,2)$

return

end

* Numbers for Crack Strain Equations

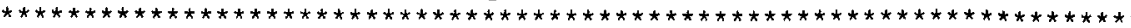

$\begin{array}{llllllllllllll}* & 5 & 10 & 15 & 20 & 25 & 30 & 35 & 40 & 45 & 50 & 55 & 60 & 65\end{array}$ 
subroutine gs ( $x i$, eta,g3,g4,g5,g6,g7,g8)

include 'ABA_PARAM. INC'

common/prps/em, gnu, glc, ac, rho, sigult, iadj1,iadj2

$a a=(2 * x i *$ eta $)$

$\mathrm{bb}=(\mathrm{xi} * \star 2-e t a * * 2-\mathrm{ac} * * 2)$

$\mathrm{cc}=(-2 * x i * e t a)$

at $f 1=a \tan 2(a a, b b)$

atf $2=a \tan 2(\mathrm{cc}, \mathrm{bb})$

cf $=x i * * 4+2 * x i * * 2 *$ eta**2-

$2 \star x i * \star 2 \star a c * \star 2+e t a * \star 4+2 * e t a * \star 2 * a c * \star 2+a c * \star 4$

$\operatorname{cf} 1=\operatorname{cf} * *(1 . / 4$.

$\operatorname{cf} 2=c f * *(3 . / 4$. $)$

$\mathrm{g} 3=\operatorname{cf} 1{ }^{*} \cos (\operatorname{atf} 1 / 2$.)

$g 4=1 / c f 1 *(e t a * \cos (a t f 2 / 2)+x i * \sin (a t f 2 / 2)$.

g5 $=$ cf $1^{*} \sin (\operatorname{atf} 1 / 2$. $)$

g5 $=$ cfa*sin (atf1/2.)

$g 6=1 / \operatorname{cf} 1 *(x i * \cos (a t f 2 / 2)-.e t a$
$g 7=a c * 2 . / \operatorname{cf} 2 * \sin (3 . / 2 . * a t f 1)$

$g 8=-(a c * * 2.) / c f 2 \star \cos \left(3.12{ }^{*} a t f 1\right)$

return

end

* Strain due to interface crack

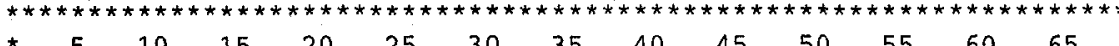

702

subroutine sevfe(xisign, xi, eta, ebxx, ebyy, ebxy, evfe)

include 'ABA_PARAM. INC'

common/xcrds/ $\mathrm{x} 1, \mathrm{x} 2, \mathrm{x} 3, \mathrm{x} 4$

common/ycrds/y1, $\mathrm{y} 2, \mathrm{y} 3, \mathrm{y} 4$

common/prps/em, gnu, g1c, ac, rho, sigult, iadj1, iadj2

common/plane/iplnstrn, iplnstrss

common/sigtau/sigo, sig1, tauo

dimension evfe (3)

call gs (xi, eta, g3,g4,g5,g6,g7,g8)

$g=e m /(2 . *(1 .+g n u))$

\section{if (iplnstrss.eq. 1 ) then}

exi1 $=1 . / \mathrm{em}^{*}\left(\operatorname{sig} 0 *\left(g 6-\mathrm{eta}{ }^{*} \mathrm{~g} 7\right)+\operatorname{sig} 1-g n u *\left(\operatorname{sig} 0 *\left(g 6+e \operatorname{ta}{ }^{*} \mathrm{~g} 7\right)\right)\right)$

exi2 $=1 . / e^{*}(2 * \tan 0 * g 4+\tan 0 *$ eta*g8-gnu* $(-\tan 0 *$ eta*g8))

eeta $1=1 . / \mathrm{em}^{*}\left(\mathrm{sig} 0 *\left(\mathrm{~g} 6+\mathrm{eta}{ }^{*} \mathrm{~g} 7\right)-g \mathrm{gu}^{*}(\mathrm{sig} 0 *(\mathrm{~g} 6-\mathrm{eta} * \mathrm{~g} 7)+\mathrm{sig} 1)\right.$

eeta $2=1 . / \mathrm{em}^{*}\left(-\tan 0^{*}\right.$ eta* $g 8-g n u^{*}\left(2^{\star} \operatorname{tau}{ }^{*} \mathrm{~g} 4+\operatorname{tau} 0^{*}\right.$ eta $\left.\left.{ }^{*} \mathrm{~g} 8\right)\right)$

exieta $1=1 . /\left(1 .{ }^{*} g\right) *\left(-\operatorname{sig} 0^{*}\right.$ eta $\left.{ }^{*} g 8\right)$

exieta $2=1 . /\left(1,{ }^{*} g\right) *\left(\right.$ tau $0 * g 6-$ tau $0 *$ eta $\left.{ }^{*} g 7\right)$ elseif (iplnstrn.eq. 1 ) then

exil $=((1 .+g n u) / e m) *((1 .-g n u) *($ sig0* $(g 6-e t a * g 7)+\operatorname{sig} 1)-$

$\#$ $g^{*}{ }^{*}\left(\operatorname{sig}^{*}\left(g 6+e \operatorname{ta}{ }^{*} \mathrm{~g} 7\right)\right)$

exi2 $=((1 .+\operatorname{tgnu}) / \mathrm{em}) *((1 .-\mathrm{gnu}) *(2 * \operatorname{tau} 0 * g 4+\operatorname{tau} 0 *$ eta*g 8$)$

$-g n u^{*}\left(-\operatorname{tau} 0 *{ }^{*} t^{*} \mathrm{~g} 8\right)$

( 1 , + gnu $) / e m) *((1,-g n u) *(\operatorname{sig} 0 *(g 6+e t a * g 7))$ $\left.-\operatorname{gnu}^{*}\left(\operatorname{sig} 0^{*}\left(\mathrm{~g} 6-\mathrm{eta}{ }^{*} \mathrm{~g} 7\right)+\operatorname{sig} 1\right)\right)$

$-\operatorname{gnu}^{*}(2 * \tan 0 * \mathrm{~g} 4+\operatorname{tau} 0 *$ eta*$\left.g 8)\right)$

$=1 . /\left(1 .{ }^{*} g\right) *(-\mathrm{sig} 0 *$ eta*g8)

evfe(1) $=\left(e x i 1+x i \operatorname{sign}^{\star} e x i 2-e b x x\right)$

evfe $(2)=($ eeta $1+x i$ sign $* e t a 2-e b y y)$

evfe $(3)=($ xisign* $($ exieta 1$)+$ exieta $2-e b x y)$

\section{return}

end

* Stress Intensity Factor

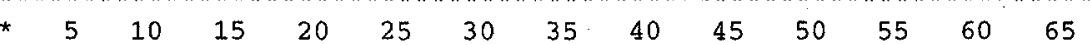

702

subroutine sif(sig0, tau0, gval, k1sign)

include 'ABA_PARAM. INC'

common/prps/em, gnu, glc, ac, rho, sigult, iadj1, iadj2

data pi / $3.141592653589793 /$

c compute the stress intensity factors

k1sign=1

spa=sqrt $(p i * a c)$

rk1 $=$ sigo*spa

if (rk1.1t.0.) then

$\mathrm{k} 1$ sign $=-1$

$r k 1=0$

endif

$r k 2=$ tauo* spa

c plane stress evaluation of the energy available for crack extension

gval $=(r k 1 * * 2+r k 2 * * 2) / e m$

return

end

* transpose (s)*det (J) 
The removed parts of this subroutine are from comment (b) of the Maple worksheet

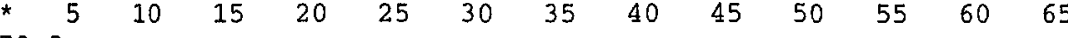

702

subroutine stj(st)

include 'ABA PARAM. INC'

common $/ x$ crds $/ x 1, x 2, x 3, x 4$

common/ycrds/y1, $2, \mathrm{y}^{3}, \mathrm{y}^{4}$

common/thck/thk1, thk 2 , thk 3 , thk 4

dimension st $(9,3)$

$$
\begin{aligned}
& t 1=x 4^{*} y 3 \\
& t 2=t 1 * t h k 4 \\
& t 3=x 1 * y 4
\end{aligned}
$$

$t 68=t 33 / 12 .+t 34 / 12 .-t 59 / 36 .-t 36 / 18,-t 37 / 36 .-t 38 / 36 .-$ $\# t 39 / 36 .-t 41 / 18 .-t 60 / 36 .+t 61 / 36,-t 62 / 36 .+t 63 / 36 .-t 64 / 36$ $\# .+t 43 / 36 .-t 44 / 18,+t 46 / 12,-t 47 / 12 .-t 48 / 36,+t 52 / 12,+t 5$

$$
\# 3 / 36 \text {. }
$$

$t 69=t 67+t 68$

$\operatorname{ST}(1,1)=t 55$

$\operatorname{ST}(1,2)=0$.

$\operatorname{ST}(9,2)=0$.

$\operatorname{ST}(9,3)=t 69$

return

end

$* \quad$ This subroutine updates the crack length

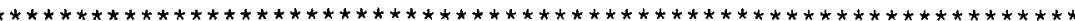

$\begin{array}{llllllllllllll}* & 5 & 10 & 15 & 20 & 25 & 30 & 35 & 40 & 45 & 50 & 55 & 60 & 65\end{array}$

702

subroutine aupdate(sig0, tau0, w, dat, deltaa)

include 'ABA_PARAM. INC'

common/thck/thk1, thk2, thk 3 , thk4

common/prps/em, gnu,g1c,ac, rho, sigult, iadj1, iadj2 call sif(sig0, tau0, gval, k1sign)

rlamda $=g 1 \mathrm{c}$

$\mathrm{rn}=1 . / 100$

$\mathrm{r} 1=0$.

if (gval.gt.r1) then

deltaa $=(($ gval $-r 1) /$ rlamda $) * *(1 . / \mathrm{rn})$

else

deltaa $=0$.

endif

if (deltaa.gt.10.) then

deltaa $=10$

endif

return

end

$\star \star \star \star * * *$

* Mass matrix

Tht

$\star \star *$ The removed parts of this subroutine are from comment $(g)$ of the Maple worksheet
$20 \quad 25$
30
35
$40 \quad 45$
55
5560
65

702

subroutine $\mathrm{mm}$ ( $\mathrm{rm}, \mathrm{rmass})$

include 'ABA_PARAM. INC'

common $/ x$ crds $/ x 1, x 2, x 3, x 4$

common/ycrds/y $1, y 2, y^{3}, y 4$

common/bdy/bx, by

common/thck/thk1, thk2, thk 3, thk4

common/prps/em, gnu, g1c, ac, rho, sigult, iadj1, iadj2

dimension $\mathrm{rm}(8,8)$

$$
\begin{aligned}
& t_{1}=t h k 4 * x 2 \\
& t 2=t 1^{*} y^{3}
\end{aligned}
$$

t3 $=$ thk $4 * \times 3$

$\mathrm{t} 46 / 7$

$t 120=t 39 / 360 \cdot D 0-t 41 / 240 \cdot D 0+t 42 / 120 \cdot D 0-t 43 / 120 \cdot D 0-t 44 / 80 \cdot D 0-$

$\# 20 . D 0-t 72 / 120 . D 0+t 49 / 240 . D 0-t 50 / 240 . D 0-t 51 / 60 . D 0+t 53 / 240 . D 0$

$t 121=-t 54 / 240 \cdot D 0-t 55 / 240.00-$

$t 57 / 120 . D 0+t 59 / 360 . D 0+t 60 / 240 . D 0+t 61$ 
$\# / 60 . D 0-t 62 / 240 . D 0+t 63 / 60 . D 0+t 64 / 20 . D 0-t 74 / 120 . D 0+t 75 / 120 . D 0$ $\mathrm{t} 123=\mathrm{t} 117+\mathrm{t} 118+\mathrm{t} 120+\mathrm{t} 121$

$\operatorname{rm}(1,1)=r h o * t 67$

$\operatorname{rm}(1,2)=r h o * 0 . D 0$

$\mathrm{rm}(1,3)=r h o * t 78$

$\operatorname{rm}(8,6)=r h o * t 116$

$\mathrm{rm}(8,7)=r h o * 0 . \mathrm{D} 0$

$\operatorname{rm}(8,8)=r h o * t 123$

\section{return}

end

$\star *$

* Body Forces Load Vector

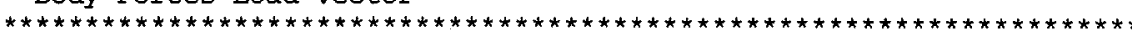
$\star \star \star *$

The removed parts of this subroutine are from comment (a) of the Maple worksheet

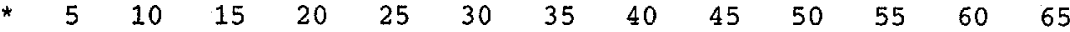

702

subroutine load( $f 3)$

include 'ABA_PARAM. INC'

common/xcrds $/ x 1, x 2, x 3, x 4$

common/ycrds/y $1, y^{2}, y^{3}, y^{4}$

common/bdy/bx, by

common/thck/thk1, thk2, thk3, thk 4

dimension $f 3(8)$

$t 1=\mathrm{Bx} *$ thk 2

$t 1=B x^{*} t h k 2$
$t 2=x \cdot y^{2}$

$t 3=t 1 * t 2$

$t 167=-t 81 / 72-t 82 / 36-t 83 / 72-t 84 / 36+t 86 / 36-t 87 / 24-t 88 / 36-$

$t 89 / 12+t 9$

$\# 0 / 36-t 91 / 36+t 94 / 12$

$t 137 / 72-t$

$\# 38 / 72+t 139 / 72-t 140 / 72+t 141 / 72$ $\begin{array}{r}f 3(1) \\ t 18 / 72+t 21\end{array}=t 3 / 24-t 6 / 36+t 8 / 72-t 10 / 24+t 12 / 36-t 15 / 72-t 16 / 72-$

$\# / 36-t 23 / 72+t 38-t 40 / 36+t 41 / 72+t 42 / 24-t 43 / 72+t 44 / 36-t 45 / 12-$ $\mathrm{t} 46 / 36-\mathrm{t} 4$

$\# 7 / 12+t 48 / 36-t 49 / 36+t 63$

t77/24

$f 3(8)=t 67 / 24+t 68 / 36-t 70 / 12+t 71 / 36+t 73 / 36+t 75 / 72-t 76 / 72-$

$\# \mathrm{t} 78 / 36+\mathrm{t} 79 / 72+\mathrm{t} 167+\mathrm{t} 95 / 72+\mathrm{t} 96 / 12-$

$t 97 / 72+t 98 / 72+t 99 / 72+t 100 / 72+t 101$

$\# / 36-t 102 / 72+t 103 / 72+t 109 / 18+t 170$

return

end

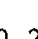
$10 \quad 15$
20
25
30.35
40
45
$55 \quad 60$

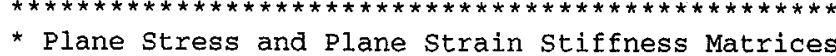

* Plane stress and Plane strain stiffness Matrices

The removed parts of this subroutine are from comment (f) of the Maple worksheet

* 70

1015

$20 \quad 25$

$30 \quad 35$

40

45

$50 \quad 55$

$60 \quad 65$

subroutine stiff(rk)

include 'ABA_PARAM. INC'

common $/ x \operatorname{crds} / x 1, x 2, x 3, x 4$

common/ycrds $/ y 1, y 2, y^{3}, y 4$

common/thck/thk1, thk2, thk 3 , thk 4

common/prps/em, gnu, g1c, ac, rho, sigult, iadj1, iadj2

common/plane/iplnstrn, iplnstrss

dimension $r k(8,8)$

double precision nu

nu=gnu

if (iplnstrss.eq.1) then

t1 $1=$ thk $2 * y^{2}$

$\mathrm{t} 2=\mathrm{thk} 1 * \mathrm{y} 2$

$t 3=\operatorname{thk} 4 * \mathrm{y} 2$

$t 1664=-t 1649 * t 91 * t 112-t 1655 * t 119 * t 112+t 1661 * t 124 * t 112$ $t 1672=-t 1649 * t 119 * t 112+t 1655 * t 154 * t 112-t 1661 * t 159 * t 112$ 
$t 1680=t 1649 * t 124 * t 112-t 1655 * t 159 * t 112-t 1661 * t 179 * t 112$

$\operatorname{rk}(1,1)=t 267 * t 11+t 275 * t 116+t 283 * t 122+t 359 * t 295+t 367 * t 300+t 375 * t 3$ \#03

$r k(1,2)=t 427 * t 295+t 435 * t 300+t 443 * t 303+t 359 * t 11+t 367 * t 116+t 375 * t 1$ $\# 22$

$+t$

$r k(8,7)=t 1591 * t 497+t 1599 * t 499+t 1607 * t 501+t 1627 * t 503+t 1635 * t 505$ \#1643*t507

$r k(8,8)=t 1664 * t 503+t 1672 * t 505+t 1680 * t 507+t 1627 * t 497+t 1635 * t 499+t$ $\# 1643 \star t 501$

elseif(iplnstrn.eq.1) then

$t 1=y 2 / 2 . D 0-y 4 / 2 . D 0$

$\mathrm{t} 2=\mathrm{x} 1{ }^{*} \mathrm{y} 2$

$t 3=x 3 * y 4$

.

$t 1451=-t 1438 * t 10 * t 23+t 1444 * t 23+t 1448 * t 18 * t 23$

$t 1459=t 1438 * t 16 * t 23+t 1443 * t 63 * t 66-t 1448 * t 16 * t 70$

$t 1466=t 1438 * t 18 * t 23-t 1444 * t 70+t 1448 * t 81 * t 66$

$r k(1,1)=t 123 * t 1+t 131 * t 25+t 138 * t 28+t 205 * t 140+t 213 * t 143+t 220 * t 146$ $r k(1,2)=t 265 * t 140+t 273^{*} t 143+t 280 * t 146+t 205 * t 1+t 213 * t 25+t 220 * t 28$ .

$\operatorname{rk}(8,7)=t 1384 * t 326+t 1392 * t 328+t 1399 * t 330+t 1417 * t 332+t 1425 * t 334$ $+t$ $\# 1432 \star t 336$

$r k(8,8)=t 1451 * t 332+t 1459 * t 334+t 1466 * t 336+t 1417 * t 326+t 1425 * t 328+t$ $\# 1432 * t 330$

endif

return

retur

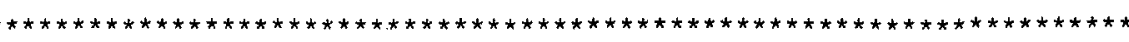
* Element Strains

Tht The removed parts of this subroutine are from comment (c) of the

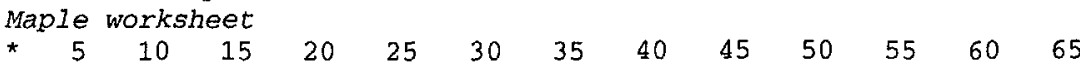

* 702

subroutine $\operatorname{strn}(e p s)$

include 'ABA_PARAM.INC'

common/disp/u1x, u1y, u2x, u2y,u3x,u3y,u4x,u4y

common $/ x c r d s / x 1, x 2, x 3, x 4$

common/ycrds $/ y 1, y 2, y^{3}, y^{4}$

common/dim/b, h

dimension eps (3), e(3)

ex $=0$
ey $=0$

ey $=0$.

$\mathrm{exy}=0$.

$x i=-1$.

eta $=0$.

in $=4$

do $10 i=1$, in

et $a=-1 .+\left(2 . /\left(\operatorname{in}^{*} 1 .+1.\right)\right) *(i * 1$.

$t 6=x 2 * y 3$

$\mathrm{t} 8=\mathrm{x} 2{ }^{*}$ eta

$t 88=-2 * t 79 * t 55+2 * t 82^{*} t 57$

$=-2 * t 79 * t 61+2 * t 82 * t 63$

tg6 $=-2 * t 79 * t 67+2 * t 82 * t 69$

$e(1)=t 53 * u 1 x+t 59 * u 2 x+t 65 * u 3 x+t 71 * u 4 x$

$e(2)=t 84^{*} u 1 y+t 88 * u 2 y+t 92 * u 3 y+t 96 * u 4 y$

$e(3)=(t 84 * u 1 x+t 53 * u 1 y+t 88 * u 2 x+t 59 * u 2 y$

$\#+t 92 * u 3 x+t 65 * u 3 y+t 96 * u 4 x+t 71 * u 4 y)$

$e x=e x+e(1)$

$e y=e y+e(2)$

$e x y=e x y+e(3)$ 
10

\section{continue}

eps $(1)=e x /(i n * 1$. $)$

eps $(2)=e y /(i n \star 1$. $)$

eps $(3)=e x y /($ in $* 1$.$) .$

\section{return}

* Element Stresses Averaged along the Interface

The removed parts of this subroutine are from comment (d) of the Maple worksheet

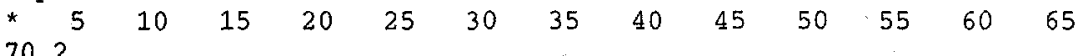

702

\section{subroutine strss (sigma)}

include 'ABA_PARAM. INC'

common/disp/u1x, u1y, u2x, u2y,u3x,u3y,u4x,u4y

common/xcrds $/ x 1, x 2, x 3, x 4$

common/ycrds/y1, y2, y3, 44

common/prps/em, gnu, glc, ac, rho, sigult, iadj1, iadj2

common/plane/iplnstrn, iplnstrss

double precision nu

dimension sigma (3), sig( 3 )

nu $=$ gnu

$\mathbf{s x}=0$.

sy $=0$.

$\mathrm{sxy}=0$.

$x i=-1$

in $=4$

do $10 i=1$, in

eta $=-1 .+\left(2 . /\left(\right.\right.$ in $\left.\left.^{*} 1 .+1.\right)\right) *(i * 1$.

if (iplnstrss.eq.1) then

$\mathrm{t} 1=\mathrm{gnu} * * 2$

$t 4=E M /(1-t 1)$ $t 97=-2 * t 84 * t 65+2 * t 87 * t 67$

$t 101=-2 * t 84 * t 71+2 * t 87 * t 73$

$t 103=t 89 * u 1 y+t 93 * u 2 y+t 97 * u 3 y+t 101 * u 4 y$

$\operatorname{sig}(1)=t 4 * t 77+t 4 * g n u * t 103$

$\operatorname{sig}(2)=t 4 * g n u * t 77+t 4 * t 103$

$\operatorname{sig}(3)=t 4 *(1 . / 2 .-g n u / 2) *(t 89 * u 1 x+t 57 * u 1 y+t 93 * u 2 x+$

$\#$ t $63 * u 2 y+t .97 * u 3 x+t 69 * u 3 y+t 101 * u 4 x+t 75 * u 4 y)$

elseif(iplnstrn.eq.1) then

$t 3=1 . /(1 .+$ gnu $)$

$\mathrm{t} 4=(1 .-\mathrm{gnu}) * \mathrm{t} 3$

$t 6=1 . /\left(1 .-2 .{ }^{*} \mathrm{gnu}\right)$

$t 116=2 \cdot{ }^{*} t 69 * t 106-2 \cdot{ }^{*} t 72 * t 108$

$t 149=1 . /\left(2 .-2 .{ }^{*} \mathrm{gnu}\right)$

$\operatorname{sig}(1)=E M *(t 4 * t 6 * t 59 * u 1 x+t 63 * g n u * t 74 * u 1 y+t 4 * t 6 * t 82 * u 2 x+t 63 * g n u$ * $t 88 * u 2 y+t 4 * t 6 * t 96 * u 3 x+t 63 * g n u * t 102 * u 3 y+t 4 * t 6 * t 110 * u 4 x+t 63 *$ gnu \#t116*u4y)

$\operatorname{sig}(2)=E M^{*}\left(t 63{ }^{*} g n u * t 59 * u 1 x+t 4 * t 6 * t 74 * u 1 y+t 63 * g n u * t 82 * u 2 x+t 4 * t 6\right.$ *t $88 * u 2 y+t 63 * g n u * t 96 * u 3 x+t 4 * t 6 * t 102 * u 3 y+t 63 * g n u * t 110 * u 4 x+t 4 * t 6 *$ $\# t 116 * u 4 y)$

$\operatorname{sig}(3)=E M^{*}\left(t 4^{*} t 149 * t 74 * u 1 x+t 4 * t 149 * t 59 * u 1 y+t 4 * t 149 * t 88 * u 2 x+t\right.$

$\# 4 * t 149 * t 82 * u 2 y+t 4 * t 149 * t 102 * u 3 x+t 4 * t 149 * t 96 * u 3 y+t 4 * t 149 * t 116 * u 4 x+t$ $\# 4 * t 149 * t 110 * u 4 y)$

endif

$\mathrm{sx}=\mathrm{sx}+\mathrm{sig}(1)$

sy $=$ sy + sig $(2)$

sxy $=\mathbf{s x y}+\operatorname{sig}(3)$

continue

$\operatorname{sigma}(1)=\operatorname{sx} /(\operatorname{in} * 1$. $)$

$\operatorname{sigma}(2)=\operatorname{sy} /\left(\operatorname{in}^{*} 1\right)$

$\operatorname{sigma}(3)=\operatorname{sxy} /(\operatorname{in} * 1$.

return

end 


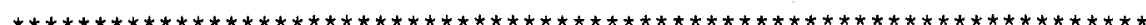
* Coefficient in Crack Strain Load Vector

The removed parts of this subroutine are from comment (e) of the Maple worksheet
$10 \quad 15$
$20 \quad 25$
$30 \quad 35$
40
$45 \quad 50 \quad 55$
$60 \quad 65$

Maple

subroutine sgaha (gaha)

include 'ABA_PARAM. INC'

common $/ x \operatorname{crds} / x 1, x 2, x 3, x 4$

common/ycrds/y1, $\mathrm{y}_{2}, \mathrm{y}^{3}, \mathrm{y}^{4}$

common/thck/thk1, thk2, thk 3 , thk 4

common/prps/em, gnu, glc, ac, rho, sigult, iadj 1, iadj2

common/plane/iplnstrn, iplnstrss

dimension gaha $(8,9)$

double precision nu

nu=gnu

if (iplnstrss.eg.1) then

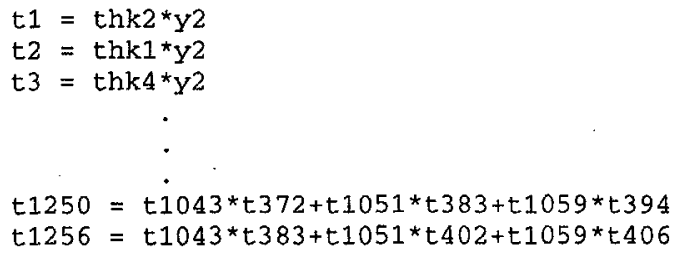

$\operatorname{GAHA}(1,1)=-t 203 * t 91 * t 112-t 239 * t 119 * t 112 * t 264 * t 124 * t 112$ GAHA $(1,2)=-t 203 * t 119 * t 112+t 239 * t 154 * t 112-t 264 * t 159 * t 112$ $\operatorname{GAHA}(1,3)=t 203 * t 124 * t 112-t 239 * t 159 * t 112-t 264 * t 179 * t 112$

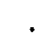

GAHA $(8,7)=-t 1250 * t 91 * t 112-t 1256 * t 119 * t 112+t 1262 * t 124 * t 112$ GAHA $(8,8)=-t 1250 * t 119 * t 112+t 1256 * t 154 * t 112-t 1262 * t 159 * t 112$ $\operatorname{GAHA}(8,9)=t 1250 * t 124^{*} t 112-t 1256 * t 159 * t 112-t 1262 * t 179 * t 112$

elseif (iplnstrn.eq.1) then

$t 1=y 2 / 2 \cdot D 0-y 4 / 2 \cdot D 0$

$t^{2}=x_{1}{ }^{*} y^{2}$

$t 3=x 3^{*} y^{4}$ $t 1044=-t 862 * t 150 / 12 . D 0+t 849 * t 243$

$\mathrm{t} 1045=\mathrm{t} 1044^{*} \mathrm{t} 16$

$t 1049=t 841 * t 236+t 857 * t 253$

$\operatorname{GAHA}(1,1)=-t 97 * t 10 * t 23+t 105 * t 23+t 120 * t 18 * t 23$

$\operatorname{GAHA}(1,2)=t 97 * t 16 * t 23+t 104 * t 63 * t 66-t 120 * t 16 * t 70$

$\operatorname{GAHA}(1,3)=t 97 * t 18 * t 23-t 105 * t 70+t 120 * t 81 * t 66$

$\operatorname{GAHA}(8,7)=-t 1039 * t 10 * t 23+t 1045 * t 23+t 1049 * t 18 * t 23$

GAHA $(8,8)=t 1039 * t 16 * t 23+t 1044 * t 63 * t 66-t 1049 * t 16 * t 70$

GAHA $(8,9)=t 1039 * t 18 * t 23-t 1045 * t 70+t 1049 * t 81 * t 66$

endif

return

end 